\title{
The Question of Negative Temperatures in Thermodynamics and Statistical Mechanics
}

\author{
David A. Lavis \\ King's College London, Department of Mathematics, London WC2R 2LS, U.K. \\ and \\ London School of Economics, Centre for Philosophy of Natural and Social Science, London WC2A 2AE, U.K.
}

\begin{abstract}
We show that both positive and negative absolute temperatures and monotonically increasing and decreasing entropy in adiabatic processes are consistent with Carathéodory's version of the second law and we explore the modifications of the Kelvin-Planck and Clausius versions which are needed to accommodate these possibilities.We show, in part by using the equivalence of distributions and the canonical distribution, that the correct microcanonical entropy, is the surface (Boltzmann) form rather than the bulk (Gibbs) form thereby providing for the possibility of negative temperatures and we counter the contention on the part of a number of authors that the surface entropy fails to satisfy fundamental thermodynamic relationships.
\end{abstract}

Keywords: negative temperatures, the Carathéodory, Kelvin-Planck and Clausius versions of the second law, microcanonical entropy.

\section{Introduction}

In most treatments of physics involving thermal considerations it is tacitly (and sometimes explicitly) assumed that the absolute temperature $T$ is non-negative. However, it is of interest to consider the contrary possibility. In this regard three questions arise:

(i) Is there evidence of such temperatures in real physical systems?

(ii) In what way does the possibility of negative temperatures impact upon the structure of thermodynamics?

(iii) What (if any) statistical mechanical models can be made to exhibit this property?

Theoretical physics, that is the development of mathematical models aiming to reflect in some way the physical reality around us, has an impetus of its own. Models are extended and generalized according to their own internal logic. ${ }^{1}$ Thus the question posed by this paper regarding negative temperatures would not be rendered pointless if there were at the moment no evidence for negative temperatures in real physical systems. ${ }^{2}$ On the other hand, if there is good evidence, it becomes necessary for this to be modelled in theoretical systems.

In Sect. 1.1 we approach an answer to (i) by first exploring the kind of properties that a system needs to have in order to exhibit negative temperatures, following this with a brief survey of the experimental systems with these properties.

It is important at this stage to highlight a difference between our account of thermodynamics in Part I and of statistical mechanics in Part II. In the former we consider both thermodynamic states, specified by controllable macroscopic

\footnotetext{
Email address: david. lavis@kcl. ac.uk (David A. Lavis)

${ }^{1}$ An example of this being the proliferation of spin and vertex models in the statistical mechanics of lattice systems (see, for example, Lavis, 2015).

${ }^{2}$ And the same would be the case with regard to negative heat capacities (see Sect. 5).
} 
variables, and also processes between them, in particular adiabatic processes, whereas our concern in the latter is solely with the possible states of the system. Thus, in respect to an answer to question (ii), the effect of negative temperatures resides not only in the thermodynamic states having this property but also in processes between them. This is of particular importance in the analysis of cycles of processes in Part I, Sect. 6, leading to the modifications of the Kelvin-Planck and Clausius versions of the second law consequent upon the cycles being between reservoirs with negative temperatures and between two reservoirs with different signs for the temperature. The last of these would, of course, involve the cycling system undergoing adiabatic processes between states where the temperatures are of opposite sign. This analysis is applied to both the cases where the cycling system and the reservoirs have positive heat capacity, meaning that entropy increases in the adiabatic processes and where the cycling system and the reservoirs have negative heat capacity, so that entropy decreases in the adiabatic processes. Whether processes between states with opposite temperature signs are possible is a question deferred to the end of Part I in Sect. 7.

Much of the recent discussion of negative temperatures in thermodynamics and statistical mechanics has centred around a dispute about the correct formula for the entropy of a thermally isolated system and the consideration of this question is a key element in the answer to (iii). In Sect. 1.2 we give a brief introduction to the elements of this dispute with a detailed account of the statistical mechanics involved presented in Part II.

In Part III we exploit the account of statistical mechanics in Part II to enter into a fuller discussion of the problems arising from the inclusion of negative temperatures in statistical mechanics and to the disputes engendered thereby, assessing the various arguments presented for the choice of either the surface or bulk entropy as the appropriate quantity for the microcanonical/micromechanical distribution. We explore the weaknesses and strengths of the cases for both the bulk and surface entropies, where the treatment of small systems has been a recurring theme. However, for the main subject of interest of this work, a more significant issue is, as we show, the inability of the bulk entropy to provide for the possibility of negative temperatures.

Our conclusions are given in Sect. 19 and supporting material is contained in Appens. A- D.

\subsection{Salient Properties of Physical Systems}

To understand the physics involved here consider a finite ${ }^{3}$ system at temperature $T$ with continuous or discrete energy spectrum in the domain $\left[E_{\min }, E_{\max }\right]$. The probability of the system having energy $E$ is $\mathrm{P}(E) \propto \omega(E) \exp (-E / T)$, where $\omega(E)$ is the density of states. ${ }^{4}$ If $\mathrm{P}(E)$ is dominated by the exponential term then, for positive temperatures, states with lower energy are more probable and, since $\mathrm{P}(E)$ must be normalizable over $\left[E_{\min }, E_{\max }\right], E_{\min }$ must be finite to prevent divergence of the normalizing sum or integral. This situation is modified if $\omega(E)$ has the effect of suppressing $\mathrm{P}(E)$ at low energies obviating the need for a lower energy bound. A similar situation occurs for negative temperatures. With the exponential factor dominating $\mathrm{P}(E)$, states with high energy are more probable and $E_{\max }$ must be finite. However, again, as we shall see in examples described below, this situation is modified when $\omega(E)$ has the effect of suppressing $\mathrm{P}(E)$ at high energies leading to convergence of the sum or integral at infinite positive energy.

To explore further we consider the simple example of a system consists of $N$ identical non-interacting atoms each with $m$ energy levels $\varepsilon_{0}<\varepsilon_{1}<\cdots<\varepsilon_{m-1}$ (where we include the possibility that $m=\infty$ ). Then

$$
E=\sum_{i=0}^{m-1} n_{i} \varepsilon_{i}, \quad N=\sum_{i=0}^{m-1} n_{i},
$$

where $n_{i}$ is the number of atoms in level $i$, and the most probable values for these distribution numbers satisfy

$$
n_{i} / n_{0}=\exp \left[\left(\varepsilon_{0}-\varepsilon_{i}\right) / T\right]
$$

\footnotetext{
${ }^{3}$ Implications of the thermodynamic limit, where the system becomes infinite, are discussed in Sect. 9.

${ }^{4}$ See the footnote on page 6 with respect to the absorption of Boltzmann's constant into the definition of temperature. For the sake of simplicity this example is formulated in terms of the canonical distribution with the system in contact with an isothermal reservoir at temperature $T$. The role of that distribution in this work, including its admission of negative temperatures, is through the equivalence of distributions, as described in Sects. 9 and 13.
} 
which, for positive $T$, is monotonically decreasing with increasing $i$. The energy $E$ has a minimum value $E_{\min }=N \varepsilon_{0}$ when each atom is in its ground state $\varepsilon_{0}$. The probability of this being the case approaches unity as $T \rightarrow+0$. The energy $E$ has, of course, from (1.2), a set of discrete values and if it is increased through this sequence from its minimum value $e^{5}$ the temperature must increase to satisfy (1.1) and (1.2). If $m=\infty$ there is no limit to the amount of energy which the system can accommodate with $T \rightarrow \infty$ as $E \rightarrow \infty$. If $m$ is finite $E_{\max }=N \varepsilon_{m-1}$. As soon as $E>N \varepsilon_{m-2}, n_{m-1}$ must be non-zero and the ratios

$$
n_{i} / n_{m-1}=\exp \left[\left(\varepsilon_{m-1}-\varepsilon_{i}\right) / T\right]
$$

are well-defined. As $E \rightarrow E_{\max }, n_{i} / n_{m-1} \rightarrow 0$, for $i=0,1, \ldots, m-2$. For (1.3) to satisfy this condition $T$ must be negative with $T \rightarrow-0$ as $E \rightarrow E_{\max }$. Somewhere along the way as $E$ increased from $E_{\min }$ to $E_{\max }$ the temperature of the system changed from positive to negative. As $E$ increases from $E_{\min }$ or decreases from $E_{\max },|T|$ increases to satisfy (1.2) or (1.3) approaching the uniform distribution $n_{i}=N / m, \forall i$, as $T \rightarrow \pm \infty$ with $E \rightarrow N\left(\varepsilon_{0}+\varepsilon_{1}+\cdots+\varepsilon_{m-1}\right) / m$, which is therefore the 'crossover' between positive and negative temperatures.

This picture of negative temperatures, situating them above $T=\infty$ rather that below $T=0$ and leading to them being regarded as 'hotter' that positive temperatures, is not restricted to this model as will be evident from our discussion in the rest of the work. ${ }^{6}$

In this simple model both $E_{\min }$ and $E_{\max }$ are finite, and the latter condition is instrumental in the occurrence of negative temperatures. However, as we have already indicated, such a bound is not necessary. All that is needed in the construction of simple models is some contribution to the density of states which applies a sufficient depression of $\mathrm{P}(E)$. In the case of the model of Matty et al. (2017, Sect. V5) this is achieved by choosing a density of states which is exponentially decreasing with energy increase. In the same spirit in Sect. 11.2 we show that if, for the $\mathfrak{B}_{m}$ model of Sect. 10.2, exponentially decreasing degeneracies are imposed on the energy levels, then the behavior of the $\mathfrak{B}_{\infty}$ model exhibits both positive and negative surface temperatures very similar to the finite states case $\mathfrak{B}_{3}$.

Notwithstanding the evidence of these rather pathological models, most experimental work yielding evidence of negative temperatures has been for systems with a finite set of discrete energy levels. In particular nuclear paramagnetic resonance experiments, where assemblies of nuclear spins, coupled to each other by spin-spin interactions, constitute a subsystem loosely coupled to a lattice. Such experiments on LiF crystals by Pound (1951) and Ramsey and Pound (1951), where a magnetic field applied to nuclear spins is rapidly reversed, have been interpreted by Purcell and Pound (1951) as leaving the system in a state which has a negative temperature. The problematic aspect of this work is in the adoption of the notion of a 'spin temperature' specific to the subsystem of nuclear spins. This, however, has been studied by Abragam and Proctor (1958), who showed that it can be defined in such a way that it is identical to the thermodynamic temperature of the lattice. They (Abragam and Proctor, 1957) also performed their own experiments on $\mathrm{LiF}$, which they describe as "the first examples of calorimetry carried out with systems at negative temperatures." With the ordering of temperatures described above with negative temperatures higher that positive temperatures, studies of nuclear antiferromagnetism in rhodium by Hakonen et al. (1993), which have reached temperatures of $280 \mathrm{pK}$ and $-750 \mathrm{pK}$, could, at the time of publication be described, respectively, as the lowest and highest temperatures ever achieved and measured. However, more recently, using a new cooling method, in which a time-varying magnetic field gradient is applied to an optically trapped cloud of cold atoms in a mixture of two internal states with different magnetic moments, Medley et al. (2011) have achieved effective spin temperatures of $\pm 50 \mathrm{pK}$.

As we have indicated, negative temperatures are most easily achieved in systems where a discrete-valued energy has a natural upper bound. The presence of kinetic energy, even in lattice systems where it is split into distinct bands makes the implementation of an upper bound a challenge, because potential and interaction energies need to be limited as well (Mosk, 2005; Rapp et al., 2010). However, this problem has been overcome by Braun et al. (2013) (see also Carr, 2013, for a discussion of this work). While the lattice of their system gave a natural upper bound to the kinetic energy by the formation of a band gap, they were able to make the potential energy negative by using an anti-trap on top of the lattice, taking the shape of an inverted parabola. They then tuned the interactions to be negative, with the net result that all three energies had an upper bound.

\footnotetext{
${ }^{5}$ It should be emphasised that the varying of $E$ with the consequential change in $T$ is not intended to represent a sequence of thermodynamic processes, but is simply a way of exploring the mathematical properties of the model

${ }^{6}$ The temperature $T_{1}$ is according to (6.4) hotter that the temperature $T_{2}$ if $1 / T_{2}>1 / T_{1}$, irrespective of the signs of $T_{1}$ and $T_{2}$.
} 


\subsection{Statistical Mechanical Considerations}

The main contenders for the entropy of a thermally isolated system are, as described in Sect. 11.1, the surface entropy and the bulk entropy. ${ }^{7}$ The salient point is that the bulk entropy is a monotonically increasing function of energy; meaning that the temperature cannot be negative. If it can be shown that the surface entropy (which is not necessarily a monotonically increasing function of energy) is unviable as an expression for the thermodynamic entropy and if no other non-monotonic candidate for the entropy is proposable then it follows that the bulk entropy is the correct choice. This is the argument made by Dunkel and Hilbert (2014a) who conclude in consequence that "consistent thermostatistics forbids negative absolute temperatures", with their position supported by Hilbert et al. (2014), Campisi (2015) and Hänggi et al. (2016). Counter arguments in favour of the surface entropy have been made by Vilar and Rubi (2014), Swendsen and Wang (2015, 2016), Wang (2015), Poulter (2016), Buonsante et al. (2016) and Abrahm and Penrose (2017). ${ }^{8}$ There are two problems with the Dunkel and Hilbert position. The first and most obvious one is that, if the experimental results are to be believed, then negative temperatures do occur in nature and if they cannot be predicted by "consistent thermostatistics", that is to say statistical mechanics, there must be something wrong with the theory. ${ }^{9}$ The second is that the arguments for the inadequacy of the surface entropy are almost all based on an analysis of systems with Hamiltonian dynamics, which, in the absence of some fairly serious modifications do not have an upper bound on the kinetic energy and do not exhibit negative temperatures. Of course, this latter point in itself does not invalidate the arguments of Dunkel and Hilbert. If the same entropy function is to be used, as one might imagine it should be, to model all thermally isolated systems in statistical mechanics, it would be necessary only to show that the surface entropy were inadequate for a system with Hamiltonian dynamics for their argument to hold. For this reason in Part II, Sects. 8-13 we have included a fairly detailed description of such systems together with an account of the discrete-energy $\mathfrak{B}_{m}$ system, which is a special case of the example used in Sect. 1.1. This allows numerical calculations for $\mathfrak{B}_{3}$, which exhibits negative temperatures, and $\mathfrak{B}_{\infty}$, where there is no upper energy bound. In the latter case the region of negative temperatures disappears unless, as indicated above and shown by the modification we subsequently make to the model, a factor is introduced in the density of states which has the effect of sufficiently depressing the surface entropy at high energies.

The argument in favour of a particular entropy form for a thermally isolated system ${ }^{10}$ is also, of course, the case for the choice of a particular thermodynamic temperature for a thermally isolated system. That being said it is appropriate at this point to describe another contender proposed by Matty et al. (2017) and Swendsen (2017a,b, 2018). They introduce the canonical distribution and, while they use it, as we do in Sect. 13, to adjudicate between the bulk and surface entropies and in favour of the latter, they go rather further. They argue (Matty et al., 2017, p. 474) "that if a system of interest has ever been in thermal contact with another system, separation will never leave either system in a quantum eigenstate. Consequently the microcanonical ensemble is not an appropriate tool for calculating thermodynamic properties of quantum systems." Although this quote is couched in terms of a quantum system they assert on the following page that the same argument applies to the classical case. There the argument is that realistic models are ones where the system is, or has been, in thermal contact with another system so that its energy $E$ is not known but fluctuates, or in the latter case has fluctuated, with the internal energy $U$ identified with $\langle E\rangle$. This is the situation we discuss in Sect. 12.1, where $\Xi_{\mathrm{A}}$ and $\Xi_{\mathrm{B}}$ are respectively our system of interest and the system with which it is in thermal contact. The energy distribution of $\Xi_{\mathrm{A}}$ is given by (12.1) and, if $\Xi_{\mathrm{B}}$ is very large relative to $\Xi_{\mathrm{A}}$ (or is an isothermal reservoir, as we have defined it in Sect. 6), this becomes the canonical distribution given by (12.10), from which the entropy is then calculated. However, they also argue (Swendsen, 2017b, p. 12) for the use of the canonical entropy even "if the system has instead been in thermal contact with a system that is the same size or

\footnotetext{
${ }^{7}$ In some of the literature the surface and bulk entropies are refer to, respectively, as the Boltzmann and Gibbs entropies. One reason to avoid this usage is to remove any confusion, as in the references in Buonsante et al. (2016), between this dispute about microcanonical entropy, with the different discussion (see, for example, Lavis, 2005, 2008) about the rival merits of the Boltzmann and Gibbs formulations of statistical mechanics.

${ }^{8}$ Dunkel and Hilbert's contention was also criticised by Frenkel and Warren (2015) and Schneider et al. (2014), with reposts in Dunkel and Hilbert (2014b) and Dunkel and Hilbert (2014c) respectively.

${ }^{9}$ It is appropriate to note at this point that Dunkel and Hilbert (2014a) argue (i) that the experimental determinations of temperature yield the surface (Boltzmann) temperature $T_{\omega}$, which can be negative, but (ii) that this is not the thermodynamic temperature, which is the positive bulk (Gibbs) temperature $T_{\Omega}$. As will be clear from our account in Part II we agree with (i) but disagree with (ii).

${ }^{10}$ Of course, the whole of our discussion is predicated on our system also being chemically isolated; that is the number $N$ of microsystems is fixed.
} 
even smaller". In fact they go further that this by supposing an historic contact with a chemical reservoir of particles of chemical potential $\mu$ (say) so that $N$ is replaced by $\langle N\rangle$, meaning that the grand canonical distribution is even better for calculating the entropy. This is, of course, standard for systems currently in contact with (large) isothermal and chemical reservoirs. And its use for 'isolated' systems is attractive since it can be shown that the canonical and grand canonical distributions are "without the weaknesses which have sparked criticism of the [surface] entropy" (Swendsen, 2017a, p. 9).

However, this account has a cost. It means that, at least in this instance, we are denying the possibility that a system can be modelled by thermal and chemical isolation with given values for $E$ and $N$; that realism demands that the model must allow for an historic thermal and chemical interactionwith the environment. This would seem to open a wider question related to the whole of model building in theoretical physics, which is based in part on simplification, the assessment of the relative importance of a range of interactions for the salient features of interest. This leads, for example, to models for the mechanics of bodies moving without friction and fluids flowing without viscosity. The supposition of thermal isolation is a simplification of this kind, the absence of which, as we shall see in Sect. 3, would make it impossible to define adiabatic processes.

\section{Part I}

\section{Thermodynamic Considerations}

\section{States and Variables}

The states of classical thermodynamics are specified by a (usually) small number of macroscopic variables, which can be divided in three ways: (i) extensive variables which scale with the size of the system and intensive variables which are invariant under scaling; (ii) independent and dependent variables, the former being controllable variables, available for manipulation by an exterior environment or experimenter and the latter responding according to the nature of the system; (iii) mechanical, used in a wide sense to include, if they are present, both magnetic and electrical variables and in contradistinction to thermal variables. Variables of mechanical extensive type are referred to as deformation variables and to each is associated an intensive variable, (pressure to volume, magnetic or electric field, respectively, to the magnetic or electric moment). One of each pair of variables is independent and controllable. In most cases the intensive mechanical variable is most easily envisaged as independent although for a fluid one can take either the pressure or volume as independent. The characteristic feature of a thermodynamic, as distinct from a mechanical, system is the presence of at least one thermal variable. A system with exactly one thermal variable is called simple $\boldsymbol{e}^{11}$ and that thermal variable can be identified as the internal energy $U$. There are many different selections of variables which can be used to specify the thermodynamic state of a system. We choose the vector $\boldsymbol{X}:=\left(\left(\boldsymbol{X}^{\mathrm{T}}, \boldsymbol{X}^{\mathrm{D}}\right)\right),{ }^{12}$ where $\boldsymbol{X}^{\mathrm{T}}$ is the $n^{\mathrm{T}}$-dimensional vector of thermal variables and $\boldsymbol{X}^{\mathrm{D}}$ is the $n^{\mathrm{D}}$-dimensional vector of deformation variables. It is convenient to use the symbol $\Xi$ to denote both the thermodynamic system and its space of states. In the latter sense $\Xi$ is an open convex set in $\mathbb{R}^{n+1}$ for some integer $n>0 .{ }^{13}$ For a simple system $n^{\mathrm{T}}=1$, $n^{\mathrm{D}}=n$ and $\boldsymbol{X}^{\mathrm{T}}$ is the scalar $X^{\mathrm{T}}=U$. Given the system $\Xi$, there exists scaled systems $\Xi(\lambda), \forall \boldsymbol{\lambda}>0$, with $\lambda \boldsymbol{X} \in \Xi(\lambda)$, $\forall \boldsymbol{X} \in \Xi$. And for two systems $\Xi_{1}$ and $\Xi_{2}$, there exists a Cartesian product system $\Xi_{1} \times \Xi_{2}$, envisaged physically as $\Xi_{1}$ and $\Xi_{2}$ placed side-by-side without any physical or mechanical interaction, with $\left(\boldsymbol{X}_{1}, \boldsymbol{X}_{2}\right) \in \Xi_{1} \times \Xi_{2}, \forall \boldsymbol{X}_{1} \in \Xi_{1}$ and $\boldsymbol{X}_{2} \in \Xi_{2}$. We shall henceforth suppose that our systems are simple or Cartesian products of simple systems, where $n^{\mathrm{T}}$ becomes the number of components of the product. The internal energy, identified as the sole thermal variable of a simple system, is a primitive quantity of thermodynamics, changes in which are given by the first law. It is without a natural conjugate intensive variable. ${ }^{14}$ Alternatively the thermal variable could be identified as the entropy $S$ with

\footnotetext{
${ }^{11}$ Lieb and Yngvason (1999, pp. 41,45) define a simple system as one which has this property together with satisfying their Axioms S-I to S-III.

${ }^{12}$ Double brackets are used to display vector components in $\Xi$ (but not the phase space $\Gamma$ ), as distinct from the representation of the elements of a Cartesian product of systems.

${ }^{13}$ Although in the case where $\Xi$ is the union of two convex subspaces separated by a planar surface (represented by the line PQ in Fig. 3) it is convenient to attach the boundary to both subspaces.

${ }^{14}$ Although, as can be seen in (4.13), thermodynamics can be formulated to make it conjugate to the inverse temperature.
} 
conjugate variable the temperature $T$, but in most accounts these are derived quantities appearing later in the analysis. Indeed, if the entropy is given as a piecewise differentiable function of the components of $\boldsymbol{X}$,

$$
\nabla S:=\left(\left(\frac{\partial S(\boldsymbol{X})}{\partial X^{\mathrm{T}}}, \frac{\partial S(\boldsymbol{X})}{\partial X^{\mathrm{D}(1)}}, \ldots, \frac{\partial S(\boldsymbol{X})}{\partial X^{\mathrm{D}(n)}}\right)\right)=\left(\left(\frac{1}{T(\boldsymbol{X})},-\frac{\xi^{(1)}(\boldsymbol{X})}{T(\boldsymbol{X})}, \ldots,-\frac{\xi^{(n)}(\boldsymbol{X})}{T(\boldsymbol{X})}\right)\right),
$$

gives the (absolute) temperature $T(\boldsymbol{X})$ and the deformation intensive variables $\xi^{(j)}(\boldsymbol{X}), j=1,2, \ldots, n{ }^{15}$ This, together with higher-order derivatives of the entropy, which yield the response functions, encapsulates the behaviour of the system. A primary task of thermodynamics is to establish the existence and properties of entropy and temperature. ${ }^{16}$ In the next section (together with accompanying appendices) we briefly outline how this is done by Lieb and Yngvason (1999) ${ }^{17}$ and by accounts, like those of Landsberg $(1956,1961)$ and Buchdahl (1966), which take as their starting point Carathéodory's statement of the second law. As we shall see, this reveals what Marsland et al. (2015) refer to as an 'ambiguity' in both formulations.

\section{Adiabatic Processes}

All processes $\boldsymbol{X} \rightarrow \boldsymbol{X}^{\prime}$ between states $\boldsymbol{X}, \boldsymbol{X}^{\prime} \in \Xi$ are a result of an outside intervention manipulating the controllable variables. ${ }^{18}$ Fundamental to any presentation of thermodynamics is a subset of processes, called adiabatic, which are defined by specific restrictions on the types of manipulations of the controllable variables which are permitted to effect the process. An assumption of thermodynamics, usually kept implicit, but made explicit by Norton (2016, p. 45) in his existence assumption and Lavis (2018, p. 139) in his hypothesis of accessibility and recoverability, is that for every pair of states $\boldsymbol{X}, \boldsymbol{X}^{\prime} \in \Xi$ there exists a process $\boldsymbol{X} \rightarrow \boldsymbol{X}^{\prime}$; some manipulation of the controllable variables can be found to take the state of the system from $\boldsymbol{X}$ to $\boldsymbol{X}^{\prime}$. Conversely, as we shall see, it is of crucial importance for thermodynamics, particularly for the second law which would be otherwise false, that there exist pairs of points $\boldsymbol{X}, \boldsymbol{X}^{\prime} \in \Xi$ for which there is no adiabatic process $\boldsymbol{X} \stackrel{\mathrm{A}}{\rightarrow} \boldsymbol{X}^{\prime}$.

The assertion that "an adiabatic process is characterized physically by the absence of any thermal interaction between the system and its environment" (Boyling, 1972, p. 36) leaves some uncertainty about what counts as a thermal interaction, or more specifically what is allowed as a 'non-thermal' interaction. According to Lieb and Yngvason $\left(1999\right.$, p. 17): $:^{19}$

A state $\boldsymbol{X}^{\prime}$ is adiabatically accessible from a state $\boldsymbol{X}$ if it is possible to change the state $\boldsymbol{X}$ to $\boldsymbol{X}^{\prime}$ by means of an interaction with some device (which may consist of mechanical and electrical parts as well as auxiliary thermodynamic systems) and a weight, in such a way that the device returns to its initial state at the end of the process whereas the weight may have changed its position in a gravitational field. Thus the only work done is by/to the weight and the loss/gain in its potential energy will be equal to the gain/loss of internal energy in the system.

\footnotetext{
${ }^{15} \mathrm{Or}$, as in the case of the pressure in a fluid, minus the intensive variable. At this point it is convenient to clarify the dimensionality of the thermodynamic variables. It is straightforward to show that, by scaling with respect to suitable constants, $T$ and $\xi^{(j)}$ can be made of the dimensions of energy $\left(\mathrm{J}:=\mathrm{m}^{2} \mathrm{~kg} \mathrm{~s}^{-2}\right)$ and $S, X^{\mathrm{D}(j)}$ and the heat capacities made dimensionless. This is the field-extensive variable representation of Lavis (2015, Sect. 1.1), where scaling for the pair $(S, T)$ is effected using Boltzmann's constant $k_{\mathrm{B}}$.

${ }^{16}$ An alternative to this is an account like that of Callen (1985) and Swendsen (2017b) based on postulates which assert the existence and properties of entropy. The entropy maximization principle in Callen's Postulate II excludes entropy decrease and his Postulate III excludes the possibility of negative temperatures thereby avoiding the ambiguities identified by Marsland et al. (2015). Postulate III was subsequently modified by Swendsen to allow for negative temperatures.

${ }^{17}$ This is the most comprehensive account of their work, with briefer versions in Lieb and Yngvason (1998) and Lieb and Yngvason (2000). The extension to non-equilibrium is given in Lieb and Yngvason (2013).

${ }^{18}$ The existence of a process $\boldsymbol{X} \rightarrow \boldsymbol{X}^{\prime}$ does not imply the passage along a sequence of (equilibrium) states in $\Xi$ from $\boldsymbol{X}$ to $\boldsymbol{X}^{\prime}$. With some exceptions (e.g. Giles, 1964) accounts of classical thermodynamics restrict the states of the system to equilibrium states, meaning that the only defined states of a process are its endpoints. As a consequence a process is specified in terms of its endpoints together with a description of the manipulations of the controllable variables used to bring it about. This point is discussed in more detail at the beginning of Sect. 4.2.

${ }^{19}$ We have adapted slightly to our notation.
} 
The salient characteristic of an adiabatic process, as stated in the first law, is contained in the last sentence in this quote; the work done on/by the system by/on its environment is equal to the increase/decrease in internal energy. Lieb and Yngvason make this specific by following Joule (1850), who used a weight falling under gravity as a measure of the work done on a system in his discussions of the mechanical equivalence of heat. Once this is done all manner of ingenuity can be used to link the falling weight to the system, leading to a variety of possible changes to the state of the system, predicated only on the linking mechanisms returning to their initial states. An obvious example is a set of levers causing the expansion or compression of a fluid. Here there is a change in a deformation variable, although a too close association between adiabatic processes and changes in deformation variables would be a mistake. A falling weight can be used to cause rubbing, stirring or electrical heating of a system without any change in the deformation variables. We should, however, note that the comment by Lieb and Yngvason (op. cit. p. 18) that:

In the usual parlance, rubbing would be an adiabatic process, but not electrical 'heating', because the latter requires the introduction of a pair of wires through the 'adiabatic enclosure'.

is at variance with Buchdahl (1966, Sect. 1.8) who asserts that:

[A system in an adiabatic enclosure] may incorporate a stirrer, or an electrical resistance through which a current may be passed from outside the enclosure, and so on. Then the passage of such a current, movement of the stirrer, variation of the deformation coordinates, are all to be counted as mechanical processes.

There is, however, a novel element in Lieb and Yngvason's definition and that is the inclusion of an "auxiliary thermodynamic system". ${ }^{20}$

An adiabatic process from $\boldsymbol{X}$ to $\boldsymbol{X}^{\prime}$ is denoted by $\boldsymbol{X} \stackrel{\mathrm{A}}{\rightarrow} \boldsymbol{X}^{\prime}$ and it is then useful, ${ }^{21}$ although not essential, to define ${ }^{22}$

$$
\begin{array}{ll}
\boldsymbol{X} \stackrel{\mathrm{A}}{\prec} \boldsymbol{X}^{\prime}:=\left\{\exists \text { at least one adiabatic process } \boldsymbol{X} \stackrel{\mathrm{A}}{\rightarrow} \boldsymbol{X}^{\prime}\right\}: & \boldsymbol{X}^{\prime} \text { is adiabatically accessible } \text { from } \boldsymbol{X}, \\
\boldsymbol{X} \stackrel{\mathrm{A}}{\nprec} \boldsymbol{X}^{\prime}:=\left\{\nexists \text { an adiabatic process } \boldsymbol{X} \stackrel{\mathrm{A}}{\rightarrow} \boldsymbol{X}^{\prime}\right\}: & \boldsymbol{X}^{\prime} \text { is adiabatically inaccessible } \text { from } \boldsymbol{X},
\end{array}
$$

and

$$
\begin{aligned}
& \boldsymbol{X} \stackrel{\mathrm{A}}{\prec} \boldsymbol{X}^{\prime}:=\quad\left\{\boldsymbol{X}^{\mathrm{A}} \prec \boldsymbol{X}^{\prime} \quad \wedge \quad \boldsymbol{X}^{\prime} \stackrel{\mathrm{A}}{\prec} \boldsymbol{X}\right\}: \quad \boldsymbol{X} \text { is adiabatically recoverable from } \boldsymbol{X}^{\prime}, \\
& \boldsymbol{X} \stackrel{\mathrm{A}}{\prec} \boldsymbol{X}^{\prime}:=\left\{\boldsymbol{X} \stackrel{\mathrm{A}}{\prec} \boldsymbol{X}^{\prime} \wedge \boldsymbol{X}^{\prime} \stackrel{\mathrm{A}}{\nprec} \boldsymbol{X}\right\}: \quad \boldsymbol{X} \text { is adiabatically irrecoverable } \text { from } \boldsymbol{X}^{\prime}, \\
& \boldsymbol{X} \stackrel{\mathrm{A}}{\mathrm{C}} \boldsymbol{X}^{\prime}:=\left\{\boldsymbol{X} \stackrel{\mathrm{A}}{\prec} \boldsymbol{X}^{\prime} \quad \vee \quad \boldsymbol{X}^{\prime} \stackrel{\mathrm{A}}{\prec} \boldsymbol{X}\right\}: \quad \boldsymbol{X} \text { is adiabatically comparable to } \boldsymbol{X}^{\prime} .
\end{aligned}
$$

In terms of this notation, let

$$
\operatorname{Cth}-\mathrm{I}(\Xi):=\left\{\forall \boldsymbol{X} \in \Xi \text { and any } \mathscr{N}_{\mathbf{x}}, \exists \boldsymbol{X}^{\prime} \in \mathscr{N}_{\mathbf{X}} \text { such that } \boldsymbol{X} \stackrel{\mathrm{A}}{\nprec} \boldsymbol{X}^{\prime}\right\},
$$

where $\mathscr{N}_{\mathbf{x}} \subset \Xi$ is an open neighbourhood of $\boldsymbol{X}$. Then we have Carathéodory's first version of the second law: ${ }^{23}$

Cth-I: Cth-I $(\Xi)$ is true for all thermodyamic systems $\Xi$.

\footnotetext{
${ }^{20}$ This lies at the heart of the proof that all Cartesian products like $\Xi_{1} \times \Xi_{2}$ of simple systems $\Xi_{1}$ and $\Xi_{2}$ are comparable. That is to say, for all pairs of states $\boldsymbol{X}_{1}, \boldsymbol{X}_{1}^{\prime} \in \Xi_{1}$ and $\boldsymbol{X}_{2}, \boldsymbol{X}_{2}^{\prime} \in \Xi_{2}$ either $\left(\boldsymbol{X}_{1}, \boldsymbol{X}_{2}\right) \stackrel{\text { A }}{\prec}\left(\boldsymbol{X}_{1}^{\prime}, \boldsymbol{X}_{2}^{\prime}\right)$ or $\left(\boldsymbol{X}_{1}^{\prime}, \boldsymbol{X}_{2}^{\prime}\right) \stackrel{\mathrm{A}}{\prec}\left(\boldsymbol{X}_{1}, \boldsymbol{X}_{2}\right)$.

${ }^{21}$ Landsberg (1961); Giles (1964); Buchdahl (1966); Lieb and Yngvason (1999).

${ }^{22}$ Lieb and Yngvason use $\prec$ to denote adiabatic accessibility, while we use $\stackrel{\text { A }}{\prec}$ to denote this more restricted accessibility, with $\boldsymbol{X} \stackrel{\text { A }}{\prec \boldsymbol{X}^{\prime}}$ and $\boldsymbol{X} \stackrel{\text { A }}{\gtrless} \boldsymbol{X}^{\prime}$ replacing $\boldsymbol{X} \succ \boldsymbol{X}^{\prime}$ and $\boldsymbol{X} \prec \prec \boldsymbol{X}^{\prime}$, respectively.

${ }^{23}$ As noted by Uffink (2001, p. 363) this is the preliminary version the truth of which, for all systems, Carathéodory (1909) proposed as the second law. He then proposes a stronger version which he adopts as his axiom II (op. cit., p. 236). The logical statement corresponding to (3.3) has been translated by Uffink; with slight changes to conform to our notation it becomes:
}

$$
\text { Cth-II }(\Xi):=\quad\left\{\forall \boldsymbol{X} \in \Xi \text { and any } \mathscr{N}_{\mathbf{X}}, \exists \boldsymbol{Y} \in \mathscr{N}_{\mathbf{X}} \text { and } \mathscr{N}_{\boldsymbol{Y}} \subset \mathscr{N}_{\mathbf{X}} \text { such that, } \forall \boldsymbol{X}^{\prime} \in \mathscr{N}_{\boldsymbol{Y}}, \boldsymbol{X}^{\mathrm{A}} \nprec \boldsymbol{X}^{\prime}\right\} .
$$

And the second version of the law Cth-II, asserting that Cth-II $(\Xi)$ is true $\forall \Xi$ replaces Cth-I. Since Cth-II $\Longrightarrow$ Cth-I, the latter is sufficient for our purposes. 


\section{Derivations of Entropy and Absolute Temperature}

It is convenient to introduce, in advance of our discussion of the derivations of the entropy $S(\boldsymbol{X})$ and temperature $T(\boldsymbol{X})$ the logical statements:

$$
\begin{aligned}
\mathrm{U}( \pm) & :=\left\{U\left(\boldsymbol{X}^{\prime}\right) \geqslant / \leqslant U(\boldsymbol{X}), \forall \boldsymbol{X} \stackrel{\mathrm{A}}{\rightarrow} \boldsymbol{X}^{\prime} \in \widetilde{\Xi} \text { such that } \boldsymbol{X}^{\mathrm{D}}=\boldsymbol{X}^{\mathrm{D}}\right\}, \\
\mathrm{S}( \pm) & :=\left\{S\left(\boldsymbol{X}^{\prime}\right) \geqslant / \leqslant S(\boldsymbol{X}), \forall \boldsymbol{X} \stackrel{\mathrm{A}}{\rightarrow} \boldsymbol{X}^{\prime} \in \widetilde{\Xi}\right\}, \\
\mathrm{T}( \pm) & :=\left\{[T(\boldsymbol{X})]^{-1} \geqslant / \leqslant 0, \forall \boldsymbol{X} \in \widetilde{\Xi}\right\},
\end{aligned}
$$

together with

$$
\mathrm{C}( \pm):=\{C(\boldsymbol{X}) \geqslant / \leqslant 0, \forall \boldsymbol{X} \in \widetilde{\Xi}\}
$$

for the heat capacity $C(\boldsymbol{X})$ at constant deformation variables, where $\widetilde{\Xi}$ may be the whole of the thermodynamic space $\Xi$ or, subject to a positive answer to question (ii) below, a subspace of $\Xi$. In principle one might suppose that $\widetilde{\Xi}$ could be any one of sixteen types correspond to the choices of signs in (4.1)-(4.4). Given that the only possible overlaps between subspaces of different types are boundaries where one or more of the conditions $U\left(\boldsymbol{X}^{\prime}\right)=U(\boldsymbol{X})$ or $S\left(\boldsymbol{X}^{\prime}\right)=S(\boldsymbol{X}), \forall \boldsymbol{X} \stackrel{\mathrm{A}}{\rightarrow} \boldsymbol{X}^{\prime}$, or $[T(\boldsymbol{X})]^{-1}=0$ or $C(\boldsymbol{X})=0 \forall \boldsymbol{X}$ apply, ${ }^{24}$ the following questions will then be addressed:

(i) Are all sixteen choices of sign thermodynamically possible?

(ii) Can subspaces of different types coexist in the same system?

(iii) Is equilibrium possible between systems or subsystems of different type?

(iv) Given that subspaces of different type can coexist in the same system are adiabatic processes possible between states within them? In particular is an adiabatic process possible between states with opposite temperature signs?

Questions (i)and (ii) are addressed in Sects. 4.1 and 4.2, and questions (iii) and (iv) in Sects. 5 and 7, respectively.

\subsection{The Lieb and Yngvason Account}

At the core of this approach is a set of axioms of which Cth-I is a consequence (see Sect. A.1). In all there are fourteen axioms (Lieb and Yngvason, 1999, pp. 88-89), ${ }^{25}$ A-I to A-VII, S-I to S-III and T-I to T-V. For present purposes in the summary in Appen. A only A-I to A-VII are required, ${ }^{26}$ although for convenience we have stated S-I as Hypotheses UAI( $\Xi)$ given in (A.2). ${ }^{27}$ Comparability and the Comparability Hypothesis ACP( $\Xi$ ) given in (A.1) play an important role in this account of thermodynamics. In particular forward sectors are nested iff $A C P(\Xi)$. Since the nesting property can be proved for simple systems (op. cit., Thm. 3.7) it follows that $\operatorname{ACP}(\Xi)$ is true for all simple systems. That the forward sector $\mathscr{F}_{\mathbf{x}}$ has a unique tangent plane $\Phi_{\mathbf{x}}$ with normal, given by (2.1), having components which are locally Lipschitz continuous with respect to $\boldsymbol{X}$ is the assumption encapsulated in Lieb and Yngvason's Axiom S-II, which also assumes that $\Phi_{\mathbf{X}}$ does not contain the thermal axis $\mathscr{L}_{\mathbf{x}}^{\text {T }}$ through $\boldsymbol{X}$. It follows that $\Xi=\Xi_{\mathbf{x}}^{(+)} \cup \Phi_{\mathbf{x}} \cup \Xi_{\mathbf{x}}^{(-)}$, where $\Xi_{\mathbf{x}}^{( \pm)}$are the open sets for which $X^{\mathrm{T} \prime} \gtrless X^{\mathrm{T}}$ on $\Xi_{\mathbf{x}}^{( \pm)} \cap \mathscr{L}_{\mathbf{x}}^{\mathrm{T}}$. Since $\mathscr{F}_{\mathbf{x}}$ is convex we have two possibilities:

(a) $\mathscr{F}_{\mathbf{X}}^{\circ} \subset \Xi^{(+)}$, when $\boldsymbol{X}$ is called a positive point,

(b) $\mathscr{F}_{\mathbf{X}}^{\circ} \subset \Xi^{(-)}$, when $\boldsymbol{X}$ is called a negative point.

\footnotetext{
${ }^{24}$ As observed in the footnote on page 5, the openness of $\widetilde{\Xi}$ is modified by the inclusion of its boundary with the other subspace or subspaces. That the boundary between positive and negative temperatures is expressed in (4.3) in terms of inverse temperature is suggested by the example in Sect. 1.1.

${ }^{25}$ We have taken the liberty of changing their Arabic numbers to Roman numerals.

${ }^{26}$ And T-I to T-III are discussed in Appendix B.

${ }^{27}$ In fact this is implied by Axiom T-IV, so Lieb and Yngvason have only thirteen independent axioms.
} 

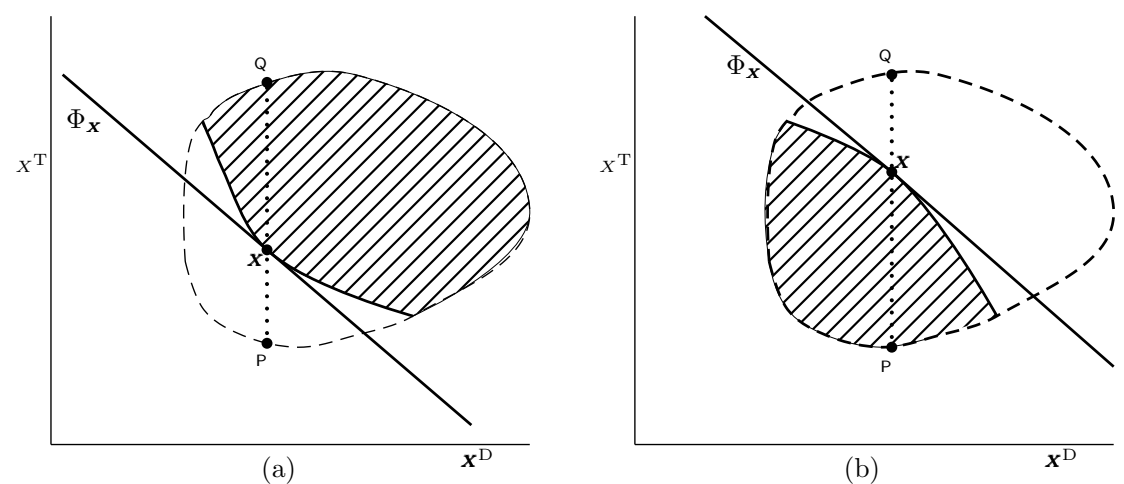

Figure 1: The forward sector $\mathscr{F}_{\mathbf{X}}$ (shaded) for (a) a p-simple system and (b) an n-simple system. The open set $\Xi$ is bounded by the broken curve and $\mathscr{F}_{\mathbf{X}}$ is relatively closed, being bounded within $\Xi$ by $\partial \mathscr{F}_{\mathbf{X}} . \mathrm{PQ}$ is the thermal axis $\mathscr{L}_{\mathbf{X}}^{\mathrm{T}}$ through $\boldsymbol{X}$. The horizontal axis signifies the $n$-dimensional space of the deformation variables. An adiabatic process from a point on $\partial \mathscr{F} \mathbf{x}$ to another point in $\Xi$ is possible if and only if that point lies in the relatively closed set $\mathscr{F} \mathbf{x}$.

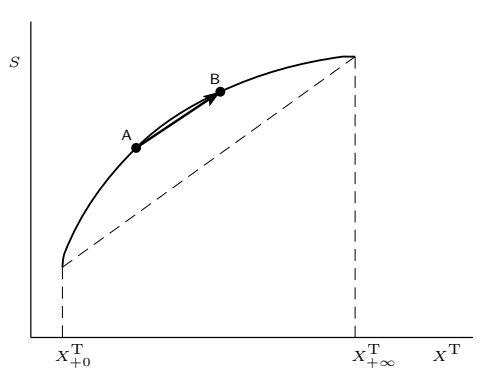

(a)

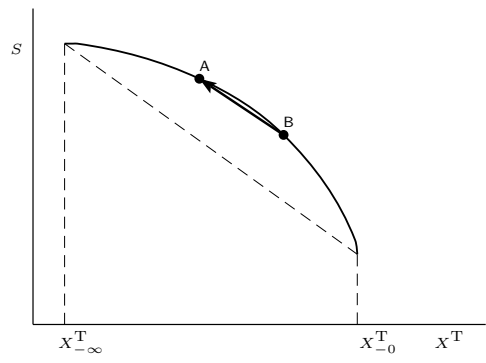

(b)

Figure 2: $S\left(\left(X^{\mathrm{T}}, \boldsymbol{X}^{\mathrm{D}}\right)\right)$ plotted against $X^{\mathrm{T}}$ for (a) a p-simple system where $\mathrm{S}(+) \wedge \mathrm{U}(+) \wedge \mathrm{T}(+)$ applies and (b) an $\mathrm{n}$-simple system where $\mathrm{S}(+) \wedge \mathrm{U}(-) \wedge \mathrm{T}(-)$ applies. The arrows denote possible adiabatic processes, $\mathrm{A}$ to $\mathrm{B}$ in (a) and $\mathrm{B}$ to $\mathrm{A}$ in (b) .

It is easy to show that if $\boldsymbol{X}$ is a positive point then any $\boldsymbol{Y} \in \mathscr{L}_{\mathbf{x}}^{\mathrm{T}}$ with $Y^{\mathrm{T}}>X^{\mathrm{T}}$ is also a positive point, and using Thm. A.1 that any $\boldsymbol{Z} \in \mathscr{L}_{\mathbf{x}}^{\mathrm{T}}$ with $Z^{\mathrm{T}}<X^{\mathrm{T}}$ is also a positive point. It follows that the whole of $\mathscr{L}_{\mathbf{x}}^{\mathrm{T}}$ consists of positive points (is a positive thermal axis) if $\boldsymbol{X}$ is a positive point, with a similar result for negative points. In their Thm. $3.3 \mathrm{where}$ they prove this result Lieb and Yngvason (op. cit.) further prove that if, for some $\boldsymbol{X} \in \Xi, \mathscr{L}_{\mathbf{x}}^{\mathrm{T}}$ is a positive/negative thermal axis then $\mathscr{L}_{\mathbf{Y}}^{\text {T }}$ is a positive/negative thermal axis for all $\boldsymbol{Y} \in \Xi$. This would then imply that $\Xi$ is of one of two types where:

- $\mathrm{U}(+)$ holds, as in Fig. 1(a), called a positive-simple (p-simple) system,

- $\mathrm{U}(-)$ holds, as in Fig. 1(b), called a negative-simple (n-simple) system.

This binary division of systems would prevent the possibility that the space $\Xi$ of a system could contain two subspaces within which $\mathrm{T}(+)$ and $\mathrm{T}(-)$ are respectively true. To understand how this dichotomy can be resolved we examine the second part of the proof of Lieb and Yngvason's Thm. 3.3. The argument is a follows. For $\boldsymbol{X}$ and $\boldsymbol{Y}$ quite close together and $\boldsymbol{X}$ a positive/negative point there is point $\partial \mathscr{F}_{\mathbf{X}} \cap \mathscr{L}_{\mathbf{Y}}^{\mathrm{T}}$ which must be positive/negative and thus $\mathscr{L}_{\mathbf{Y}}^{\mathrm{T}}$ is a positive/negative thermal axis. So the thermal axes of $\Xi$ are either all positive or all negative. It follows that in order for $\Xi$ to contain both positive and negative thermal axes it must be the union $\Xi^{(\mathrm{P})} \cup \Xi^{(\mathrm{N})}$ of a p-simple and an n-simple system, each with its own nested set of forward sectors, where $\Xi^{(\mathrm{P})}$ and $\Xi^{(\mathrm{N})}$ are convex and the interface is planar and parallel to the thermal axis. as shown in Fig. 3. 


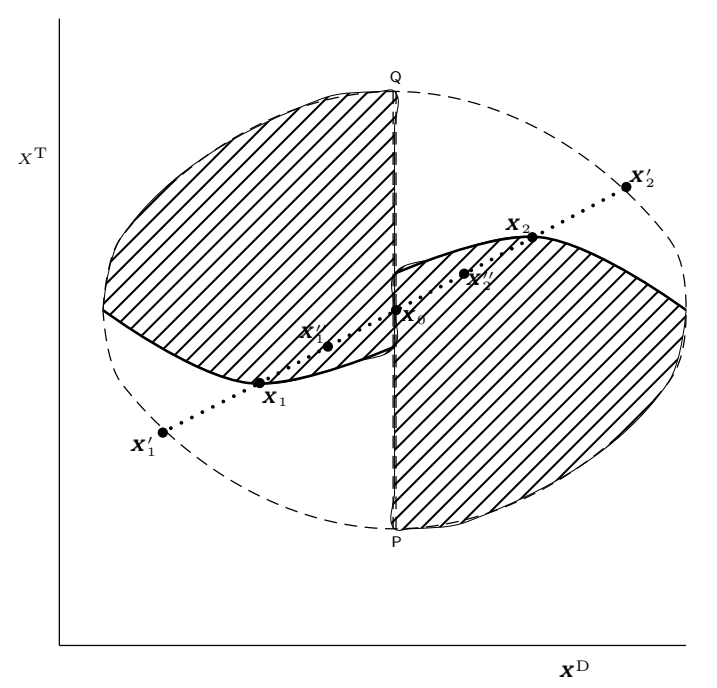

Figure 3: A system where $\Xi:=\Xi^{(\mathrm{P})} \cup \Xi^{(\mathrm{N})}$ The plane PQ separates the p-simple system $\Xi^{(\mathrm{P})}$ on the left from the n-simple system $\Xi^{(\mathrm{N})}$ on the right. The labelling of states on the dotted line is the same as in Fig. 6.

Before investigating the geometry of this situation we consider further the properties of a single p-simple or n-simple system for which Lieb and Yngvason's account (see Appen. A.2) provides a definition of entropy $S(\boldsymbol{X})$ satisfying $\mathrm{S}(+)$; that is the law of entropy increase, meaning that entropy increases upwards along the positive thermal axis in Fig. 1(a) and downwards along the negative thermal axis in Fig. 1(b) and the boundaries of forward sectors are adiabats. Concavity of the entropy function $S(\boldsymbol{X})$ then follows from $\mathrm{S}(+)$ and Axiom A-VII so that the heat capacity at constant deformation variables is positive; $\mathrm{C}(+)$ is true. However, none of the structure of Lieb and Yngvason's work is able to decide between systems being p-simple or n-simple. They simply observe (op. cit., p. 47, their italics) that:

As far as our axiomatic framework is concerned the direction of the energy coordinate and hence of the forward sectors is purely conventional, except for the proviso that once it has been set for one system it is set for all systems. ... We shall adopt the convention that they are on the positive energy side. From a physical point of view there is more at stake, however. In fact, our operational interpretation of adiabatic processes ... involves either the raising or lowering of a weight in a gravitational field and these two cases are physically distinct. Our convention, together with the usual convention for the sign of energy for mechanical systems and energy conservation, means that we are concerned with a world where adiabatic process at fixed work coordinate can never result in the raising of a weight, only in the lowering of a weight. The opposite possibility differs from the former in a mathematically trivial way, namely by an overall sign of the energy, but given the physical interpretation of the energy direction in terms of raising and lowering of weights, such a world would be different from the one we are used to.

Meaning that they have opted to concentrate on the "world" of p-simple systems. Their formal definition of temperature is introduced at quite a late stage in their paper, but it is preceded (op. cit. p. 44) by the familiar definition (see (2.1)) that

$$
T(\boldsymbol{X}):=\left(\frac{\partial S(\boldsymbol{X})}{\partial X^{\mathrm{T}}}\right)^{-1}
$$

predicated on the condition that entropy "is sufficiently smooth in order to make sense of [(4.5)]." It follows that $\mathrm{S}(+) \wedge \mathrm{U}( \pm) \Longrightarrow \mathrm{T}( \pm)$ so that a conclusion of Lieb and Yngvason's development is that a system of a single type satisfies:

$$
\mathrm{LY}:=\{\mathrm{S}(+) \wedge\{[\mathrm{U}(+) \wedge \mathrm{T}(+)] \vee[\mathrm{U}(-) \wedge \mathrm{T}(-)]\}\}
$$




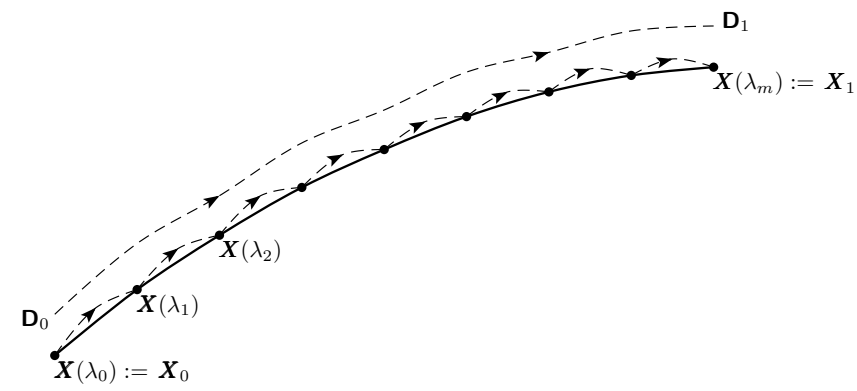

Figure 4: Comparison of delimitation and demarcation. The curve contains the endpoints of a sequence of processes, shown as broken lines, which are demarcated by the curve as proposed by Lavis (2018). The curve from $\mathrm{D}_{0}$ to $\mathrm{D}_{1}$ is representative of the set of curves which are delimited by the curve as proposed by Norton (2016).

It should be emphasised that this statement is a consequence of the formal structure of Lieb and Yngvason's approach, neglecting their informal argument for choosing p-simple systems. That this results in two types of systems is regarded by Marsland et al. (2015) as an ambiguity in this approach. ${ }^{28}$

The case where $\Xi$ consists of p-simple and n-simple subsystems, where (with $\mathrm{S}(+)$ true) $T>0$ and $<0$ respectively, is schematically shown in Fig. 3. The entropy increases upwards in the p-simple subsystem on the left and downwards in the n-simple subsystem on the right. The heavy lines are forward sector boundaries, that is adiabats. States $\boldsymbol{X}_{1}$ and $\boldsymbol{X}_{2}$ could be identified with $\boldsymbol{X}_{\mathrm{B}}$ and $\boldsymbol{X}_{\mathrm{C}}$ which feature in the T $( \pm)$ cycle in Sect. 6 and for which $S\left(\boldsymbol{X}_{\mathrm{B}}\right)=S\left(\boldsymbol{X}_{\mathrm{C}}\right)$. They are not connected by an adiabat. The dotted line through $\boldsymbol{X}_{1}^{\prime}$ and $\boldsymbol{X}_{2}^{\prime}$ could be regarded as a representation of the entropy curves in Figs. 2 (a) and (b) or equivalently the first half of the curve in Fig. 6 with the same labelling of states (if $\Xi$ were a union of all four subsystems). Entropy increases from $\boldsymbol{X}_{1}^{\prime}$ to $\boldsymbol{X}_{0}$ and from $\boldsymbol{X}_{2}^{\prime}$ to $\boldsymbol{X}_{0}$. At $\boldsymbol{X}_{0}$ where the line crosses $\mathrm{PQ}$, and indeed over the whole of $\mathrm{PQ}, 1 / T(\boldsymbol{X})=0$.

\subsection{The Approach Based Directly on Carathéodory's Version of the Second Law}

Most versions of thermodynamics rely on a certain level of continuity and differentiability of thermodynamic functions with appropriate caveats applied in critical regions. The approach of Lieb and Yngvason is almost free of such analytic assumptions ${ }^{29}$ and also of discussion of processes related to curves in $\Xi$. However, the standard argument (Buchdahl, 1966, Chap. 5) for deriving the existence of entropy and temperature from Carathéodory's version of the second law Cth-I relies heavily on differential forms yielding curves in $\Xi$, which are then taken to represent 'equilibrium processes'.

Here a little caution is needed. As has been pointed out by many authors (Cooper, 1967; Norton, 2016; Lavis, 2018), the proposition that a curve in the space of thermodynamic states can represent an 'equilibrium' or 'quasi-static' process, with the state of the system remaining in equilibrium as it traverses the points of the curve, is oxymoronic. The initiation of any process necessarily involves the manipulation of the controllable variables which will drive the system from equilibrium with the return to an equilibrium state occurring only after a 'leave-it-alone' stage (Wallace, 2014), which allows it to settle down. Equilibrium processes are thus (Cooper, 1967, p. 174) "either a contradiction in terms or limits of processes through non-equilibrium states which cannot be described in terms of equilibrium theory." There have been two recent proposals for reinterpretation. In the first of these, called delimitation, Norton (op.cit.) envisaged (rather in the manner suggested by Cooper) that an equilibrium process was in fact, as shown in the broken line $\mathrm{D}_{0}$ to $\mathrm{D}_{1}$ in Fig. 4 a sequence of non-equilibrium states near to the curve. ${ }^{30}$ In the second, called demarcation,

\footnotetext{
${ }^{28}$ In our discussion of the approach based on Carathéodory's in Sect. 4.2 we see that this is compounded in the eyes of Marsland et al. by a further 'ambiguity' in relation to the direction of entropy change in adiabatic processes.

${ }^{29}$ Except, as we have indicated above, their axiom S-II, which proposes that the slope of the tangent plane to an adiabatic hypersurface is locally Lipschitz continuous.

${ }^{30}$ The problems associated with the invocation of 'non-equilibrium states' and 'nearness' are discussed by Lavis(op. cit.).
} 
Lavis (op. cit.) proposed, as shown in Fig. 4, that an equilibrium process from $\boldsymbol{X}_{0}$ to $\boldsymbol{X}_{1}$ along a curve is understood to be the set of all sequences of processes $\boldsymbol{X}_{0}=: \boldsymbol{X}\left(\lambda_{0}\right) \rightarrow \boldsymbol{X}\left(\lambda_{1}\right) \rightarrow \cdots \rightarrow \boldsymbol{X}\left(\lambda_{m}\right):=\boldsymbol{X}_{1}$ for all $m$, where the endpoints of the processes lie on the curve. In this section and throughout this work we shall adopt the demarcation interpretation of equilibrium processes.

According to the first law of thermodynamics, if, for $\boldsymbol{X}, \boldsymbol{X}^{\prime} \in \Xi, \boldsymbol{X}^{\prime}$ is adiabatically accessible from $\boldsymbol{X}$, that is $\boldsymbol{X} \stackrel{\text { A }}{\prec} \boldsymbol{X}^{\prime}$, then there exists an adiabatic work function $W^{(\mathrm{A})}\left(\boldsymbol{X}, \boldsymbol{X}^{\prime}\right)$, which satisfies appropriate transitivity, additivity and scaling properties and which measures the amount of work done by the system to implement any adiabatic process $\boldsymbol{X} \stackrel{\mathrm{A}}{\rightarrow} \boldsymbol{X}^{\prime}{ }^{31}$ This means that the work done by the system during an adiabatic process depends only on its endpoints ( Giles 1964, p. 15; Buchdahl 1966, p. 40) and it can be shown (Boyling, 1972, p. 38) that $^{32}$

$$
W^{(\mathrm{A})}\left(\boldsymbol{X}, \boldsymbol{X}^{\prime}\right)=X^{\mathrm{T}}-X^{\mathrm{T} \prime}
$$

Particularly in connection with the use of this result in Sects. 6 and 7, we note that: ${ }^{33}$

(i) Not all work performed on the system involves a variation of the deformation coordinates, since "[an adiabatic enclosure] may incorporate a stirrer, or an electric resistance through which a current may be passed from outside the enclosure" (Buchdahl, 1966, Sect. 8).

(ii) Given that here we are considering only one subspace of the decomposition like that shown in Fig. 3 or equivalently that $\Xi$ consists of only one such subspace, then we suppose that if, for two states $\boldsymbol{X}, \boldsymbol{X}^{\prime} \in \Xi, S(\boldsymbol{X})=S\left(\boldsymbol{X}^{\prime}\right)$ then there exists an adiabat (a curve of constant entropy) in $\Xi$ connecting $\boldsymbol{X}$ and $\boldsymbol{X}^{\prime}$; that is to say is a curve which demarcates sequences of recoverable adiabatic processes from $\boldsymbol{X}$ to $\boldsymbol{X}^{\prime}$.

(iii) If $\boldsymbol{X}^{\prime}:=\boldsymbol{X}+\Delta \boldsymbol{X}$, where $\Delta \boldsymbol{X}:=\left(\left(\Delta \boldsymbol{X}^{\mathrm{T}}, \Delta \boldsymbol{X}^{\mathrm{D}}\right)\right),|\Delta \boldsymbol{X}|$ is small and $W^{(\mathrm{A})}\left(\boldsymbol{X}, \boldsymbol{X}^{\prime}\right)=-\boldsymbol{\xi}(\boldsymbol{X}) \cdot \Delta \boldsymbol{X}^{\mathrm{D}}$, where $\boldsymbol{\xi}(\boldsymbol{X})$ is the vector of intensive variables in (2.1), then, from (4.7),

$$
\triangle X^{\mathrm{T}}-\boldsymbol{\xi}(\boldsymbol{X}) \cdot \Delta \boldsymbol{X}^{\mathrm{D}}=0
$$

and an adiabatic process $\boldsymbol{X}^{\prime} \stackrel{\mathrm{A}}{\rightarrow} \boldsymbol{X}$ also exists, that is $\boldsymbol{X} \stackrel{\mathrm{A}}{\prec} \boldsymbol{X}^{\prime} ; \boldsymbol{X}$ is recoverable from $\boldsymbol{X}^{\prime}$.

(iv) The same mechanisms of implementation apply to the work done in a non-adiabatic process $\boldsymbol{X} \rightarrow \boldsymbol{X}^{\prime}$, when (4.7) is replaced by

$$
W=X^{\mathrm{T}}-X^{\mathrm{T} \prime}+Q
$$

where $Q \neq 0$ is the heat received by the system. (This is effectively a definition of heat transfer.) Unlike the adiabatic work function, neither work nor heat is a function of the pair $\left(\boldsymbol{X}, \boldsymbol{X}^{\prime}\right)$ since different proportions of each can feature in the process $\boldsymbol{X} \rightarrow \boldsymbol{X}^{\prime}$.

Relating this analysis of adiabatic processes to the mathematics of Appen. B by setting $\xi^{(0)}(\boldsymbol{X}):=-1$, it follows that a $\mathcal{D}(\boldsymbol{\xi})$-conforming curve $\mathscr{L}\left(\boldsymbol{X}_{0}, \boldsymbol{X}_{1}\right)$, that is one on which

$$
\mathrm{d} X^{\mathrm{T}}-\boldsymbol{\xi}(\boldsymbol{X}) \cdot \mathbf{d} \boldsymbol{X}^{\mathrm{D}}=0 \quad \forall \quad\left(\left(\mathrm{d} X^{\mathrm{T}}, \mathbf{d} \boldsymbol{X}^{\mathrm{D}}\right)\right),
$$

demarcates sequences of recoverable adiabatic processes. In the terminology adopted by Lavis (2018) such curves are called reversible. If $\boldsymbol{X}$ and $\boldsymbol{X}^{\prime}$ are connected by a $\mathcal{D}(\boldsymbol{\xi})$-conforming curve then $\boldsymbol{X} \stackrel{\mathrm{A}}{\prec \boldsymbol{X}^{\prime}}$ and it follows from Cth-I, that $\forall \boldsymbol{X} \in \Xi$ and any $\mathscr{N}_{\mathbf{X}}, \exists \boldsymbol{X}^{\prime} \in \mathscr{N}_{\mathbf{X}}$ which cannot be connected to $\boldsymbol{X}$ by a $\mathcal{D}(\boldsymbol{\xi})$-conforming curve. In most presentations

\footnotetext{
${ }^{31}$ Thus corresponding to the work done by the system on its environment. This simply amounts to a convenient choice of sign for $W^{(\mathrm{A})}\left(\boldsymbol{X}, \boldsymbol{X}^{\prime}\right)$

${ }^{32}$ In fact the proof demands that $\Xi$ is an adiabatically directed set, a condition which Boyling incorporates into his statement of the first law.

${ }^{33}$ In these observations it is convenient to preempt the derivation of the existence of entropy later in this section.
} 
(Buchdahl, 1966, p. 70) this is taken (by way of Thm. B.1) to justify the assertion that there exists a function $\lambda(\boldsymbol{X})$ so that $\mathcal{D}(\boldsymbol{\xi} / \lambda)$ is an exact differential. ${ }^{34}$ That is, there exists a function $\sigma(\boldsymbol{X})$ such that

$$
\lambda(\boldsymbol{X}) \mathrm{d} \sigma(\boldsymbol{X})=\mathrm{d} X^{\mathrm{T}}-\boldsymbol{\xi}(\boldsymbol{X}) \cdot \mathbf{d} \boldsymbol{X}^{\mathrm{D}}, \quad \forall \quad \boldsymbol{X} \text { and }\left(\left(\mathrm{d} X^{\mathrm{T}}, \mathbf{d} \boldsymbol{X}^{\mathrm{D}}\right)\right) .
$$

The argument which replaces $\sigma(\boldsymbol{X})$ with the entropy $S(\boldsymbol{X})$ with the right additive and scaling properties and $\lambda(\boldsymbol{X})$ by the absolute temperature $T(\boldsymbol{X})$, so that,

$$
T(\boldsymbol{X}) \mathrm{d} S(\boldsymbol{X})=\mathrm{d} X^{\mathrm{T}}-\boldsymbol{\xi}(\boldsymbol{X}) \cdot \mathbf{d} \boldsymbol{X}^{\mathrm{D}}
$$

is standard (see e.g. Buchdahl, 1966, Chap. 5) and will not detain us here. We conclude from (4.10) that reversible curves, that is curves which demarcate sequences of adiabatic recoverable processes, are adiabats. ${ }^{35}$

We pause to note that (4.12) expressed in the form

$$
\mathrm{d} S\left(\left(X^{\mathrm{T}}, \boldsymbol{X}^{\mathrm{D}}\right)\right)=\frac{1}{T(\boldsymbol{X})} \mathrm{d} X^{\mathrm{T}}-\frac{\boldsymbol{\xi}(\boldsymbol{X})}{T(\boldsymbol{X})} \cdot \mathbf{d} \boldsymbol{X}^{\mathrm{D}}
$$

is equivalent to (2.1). However, (4.12) in the form

$$
\mathrm{d} X^{\mathrm{T}}=T(\boldsymbol{X}) \mathrm{d} S(\boldsymbol{X})+\boldsymbol{\xi}(\boldsymbol{X}) \cdot \mathbf{d} \boldsymbol{X}^{\mathrm{D}}
$$

implies a similar form for the gradient of $X^{\mathrm{T}}$ only if $S\left(\left(X^{\mathrm{T}}, \boldsymbol{X}^{\mathrm{D}}\right)\right)$ is a monotonic function of $X^{\mathrm{T}}$, when $S=S\left(\left(X^{\mathrm{T}}, \boldsymbol{X}^{\mathrm{D}}\right)\right)$ can be inverted to give $X^{\mathrm{T}}=X^{\mathrm{T}}\left(\left(S, \boldsymbol{X}^{\mathrm{D}}\right)\right)$. This condition is that the system is, for all its parameter values, either p-simple or $\mathrm{n}$-simple, which has been assumed for the present system or for the subspace of $\Xi$ to which we have confined ourselves. In the account of Lieb and Yngvason entropy is defined in such a way as to satisfy (A.5), implying that entropy does not decrease in an adiabatic process and, as we have seen above and as is confirmed by (4.12), with $\mathbf{d} \boldsymbol{X}^{\mathrm{D}}=\mathbf{0}$, the internal energy $U\left(:=X^{\mathrm{T}}\right)$ increases along the thermal axis if $T(\boldsymbol{X})>0$ and decreases if $T(\boldsymbol{X})<0$. However, in the account in this section there is no a priori argument for entropy increase and the full range of possibilities is revealed by considering either entropy $S$ to be an independent variable with the internal energy $U$ as a dependent variable (with corresponding differential form (4.14)) or vice-versa (with corresponding differential form (4.13)):

\subsubsection{Approach I: Treating Entropy $S$ as an Independent Variable}

The states of $\Xi$ are now specified by $\tilde{\boldsymbol{X}}:=\left(\left(S, \boldsymbol{X}^{\mathrm{D}}\right)\right)$ and we want to know if $\left(\left(S_{1}, \boldsymbol{X}_{1}^{\mathrm{D}}\right)\right)=: \tilde{\boldsymbol{X}}_{1} \stackrel{\mathrm{A}}{\prec} \tilde{\boldsymbol{X}}_{2}:=\left(\left(S_{2}, \boldsymbol{X}_{2}^{\mathrm{D}}\right)\right)$. Since it is always possible for a line of constant entropy to demarcate a sequence of adiabatic processes, $\left(\left(S_{1}, \boldsymbol{X}_{1}^{\mathrm{D}}\right)\right) \stackrel{\mathrm{A}}{\prec}$ $\left(\left(S_{2}, \boldsymbol{X}_{2}^{\mathrm{D}}\right)\right) \Longleftrightarrow\left(\left(S_{1}, \boldsymbol{X}_{1}^{\mathrm{D}}\right)\right) \stackrel{\mathrm{A}}{\prec}\left(\left(S_{2}, \boldsymbol{X}_{1}^{\mathrm{D}}\right)\right)$. It is reasonable to assume that the set of states on $\mathscr{L}_{1}:=\left\{\boldsymbol{X} \mid \boldsymbol{X}^{\mathrm{D}}=\boldsymbol{X}_{1}^{\mathrm{D}}\right\}$ adiabatically accessible from $\boldsymbol{X}_{1}$ is a closed interval; that is $S_{2} \in\left[S_{1 \mathrm{~L}}, S_{\mathrm{IU}}\right]$, for some $S_{\mathrm{IL}}<S_{\mathrm{IU}}$. Suppose that $S_{1}$ is an interior point of $\left[S_{1 \mathrm{~L}}, S_{1 \mathrm{U}}\right]$. Then $\exists \varepsilon>0$ such that $\left(\left(S_{1}, \boldsymbol{X}_{1}^{\mathrm{D}}\right)\right) \stackrel{\mathrm{A}}{\prec}\left(\left(S_{1} \pm \Delta S, \boldsymbol{X}^{\mathrm{D} \prime}\right)\right), \forall \boldsymbol{X}^{\mathrm{D} \prime}$ and $0<\triangle S<\varepsilon$. Given that this accessibility can, by means of sequences of processes along adiabats, be extended into all points in a neighbourhood of $\left(\left(S_{1}, \boldsymbol{X}^{\mathrm{D}} 1\right)\right)$, Cth-I is contradicted and thus $S_{1}=S_{\mathrm{LL}}$ or $S_{1}=S_{\mathrm{IU}}$. It follows that $\mathscr{L}_{1}$ is covered by two sets $\mathcal{J}_{\mathrm{L}}$ and $\mathcal{J}_{\mathrm{U}}$ of closed intervals, those of points adiabatically accessible from their lower and upper bounds respectively. It is clear that, for $S_{\mathrm{A}}<S_{\mathrm{D}}$,

$$
\begin{aligned}
& {\left[S_{\mathrm{A}}, S_{\mathrm{B}}\right] \in \mathcal{J}_{\mathrm{L}} \wedge\left[S_{\mathrm{C}}, S_{\mathrm{D}}\right] \in \mathcal{J}_{\mathrm{U}} \quad \Longrightarrow \quad\left[S_{\mathrm{A}}, S_{\mathrm{B}}\right] \cap\left[S_{\mathrm{C}}, S_{\mathrm{D}}\right]=\emptyset} \\
& {\left[S_{\mathrm{A}}, S_{\mathrm{B}}\right] \in \mathcal{J}_{\mathrm{X}} \wedge\left[S_{\mathrm{C}}, S_{\mathrm{D}}\right] \in \mathcal{J}_{\mathrm{X}} \wedge\left[S_{\mathrm{A}}, S_{\mathrm{B}}\right] \cap\left[S_{\mathrm{C}}, S_{\mathrm{D}}\right] \neq \emptyset \Longrightarrow\left[S_{\mathrm{A}}, S_{\mathrm{D}}\right] \in \mathcal{J}_{\mathrm{X}}, \quad \mathrm{X}=\mathrm{L}, \mathrm{U} .}
\end{aligned}
$$

It follows from this that, the covering must consist only of half-infinite members of $\mathcal{J}_{\mathrm{L}}$ or half-infinite members of $\mathcal{J}_{U}$. That is,

$$
\left(\left(S_{1}, \boldsymbol{X}_{1}^{\mathrm{D}}\right)\right) \stackrel{\mathrm{A}}{\prec}\left(\left(S_{2}, \boldsymbol{X}_{2}^{\mathrm{D}}\right)\right) \Longleftrightarrow \begin{cases}S_{1} \leq S_{2}: & \text { (Assertion } \mathrm{S}(+)), \\ \text { or } & \\ S_{1} \geq S_{2}: & \text { (Assertion } \mathrm{S}(-)),\end{cases}
$$

\footnotetext{
${ }^{34}$ In fact, as we see in Appen. B the correct condition is slightly more stringent and given by Carathéodory' local theorem (Thm. B.2); the curve $\mathscr{L}\left(\boldsymbol{X}, \boldsymbol{X}^{\prime}\right)$ must lie entirely in $\mathscr{N}_{\mathbf{X}}$. (Condition (B.1) is automatically satisfied since $\xi^{(0)}(\boldsymbol{X})=1$.) On the other hand, it is of course the case that, when $n=1$, the differential form is always integrable. Cth-I and Thm. B.1 are not required to establish (4.11).

${ }^{35}$ This is, of course, otherwise obvious in the Lieb and Yngvason approach.
} 
and it is easy to see that, for any system $\Xi$, either the entropy increasing condition $S(+)$ or the entropy decreasing condition S(-) applies for all adiabatic processes.

\subsubsection{Approach II: Treating Internal Energy $U$ as an Independent Variable}

The states of $\Xi$ are now specified, as in all our previous discussion, by $\boldsymbol{X}:=\left(\left(U, \boldsymbol{X}^{\mathrm{D}}\right)\right)$ and we want to know whether $\left(\left(U_{1}, \boldsymbol{X}_{1}^{\mathrm{D}}\right)\right)=: \boldsymbol{X}_{1} \stackrel{\mathrm{A}}{\prec} \boldsymbol{X}_{2}:=\left(\left(U_{2}, \boldsymbol{X}_{2}^{\mathrm{D}}\right)\right)$. Here the only decidable case is when $\boldsymbol{X}_{1}^{\mathrm{D}}=\boldsymbol{X}_{2}^{\mathrm{D}}$ and we consider the accessibility condition $\left(\left(U_{1}, \boldsymbol{X}_{1}^{\mathrm{D}}\right)\right)=: \boldsymbol{X}_{1} \stackrel{\mathrm{A}}{\prec} \boldsymbol{X}_{2}^{\prime}:=\left(\left(U_{2}, \boldsymbol{X}_{1}^{\mathrm{D}}\right)\right)$. Again we assume that the set of states on $\mathscr{L}_{1}:=\left\{\boldsymbol{X} \mid \boldsymbol{X}^{\mathrm{D}}=\boldsymbol{X}_{1}^{\mathrm{D}}\right\}$ accessible from $\boldsymbol{X}_{1}$ is a closed interval; in this case $U_{2} \in\left[U_{\mathrm{lL}}, U_{1 \mathrm{U}}\right]$, for some $U_{\mathrm{IL}}<U_{1 \mathrm{U}}$. Following Approach I we now need to show that $U_{1}$ cannot be an interior point of $\left[U_{\mathrm{IL}}, U_{\mathrm{lU}}\right]$. If this were the case then there would exist $\varepsilon>0$ with $\left(\left(U_{1}, \boldsymbol{X}_{1}^{\mathrm{D}}\right)\right) \stackrel{\mathrm{A}}{\prec}\left(\left(U_{1} \pm \triangle U, \boldsymbol{X}_{1}^{\mathrm{D}}\right)\right), \forall 0<\triangle U<\varepsilon$. But this is not sufficient to contradict Cth-I. For that we need $\left(\left(U_{1}, \boldsymbol{X}_{1}^{\mathrm{D}}\right)\right) \stackrel{\mathrm{A}}{\prec}\left(\left(U_{1} \pm \triangle U, \boldsymbol{X}_{1}^{\mathrm{D}}+\triangle \boldsymbol{X}^{\mathrm{D}}\right)\right), \forall 0<\left|\left(\left(\triangle U, \triangle \boldsymbol{X}^{\mathrm{D}}\right)\right)\right|<\boldsymbol{\varepsilon}$. A necessary and sufficient condition for this is that $\exists\left(\left(U^{\prime}, \boldsymbol{X}_{1}^{\mathrm{D}}\right)\right)$, with $U^{\prime} \in\left[U_{\mathrm{IL}}, U_{\mathrm{lU}}\right]$, such that $\left(\left(U^{\prime}, \boldsymbol{X}_{1}^{\mathrm{D}}\right)\right) \stackrel{\mathrm{A}}{\prec}\left(\left(U_{1} \pm \triangle U, \boldsymbol{X}_{1}^{\mathrm{D}}+\triangle \boldsymbol{X}^{\mathrm{D}}\right)\right)$. With this rather weak assumption the rest of the argument parallels that for Approach I and leads to

$$
\left(\left(U_{1}, \boldsymbol{X}_{1}^{\mathrm{D}}\right)\right) \stackrel{\mathrm{A}}{\prec}\left(\left(U_{2}, \boldsymbol{X}_{1}^{\mathrm{D}}\right)\right) \Longleftrightarrow \begin{cases}U_{1} \leq U_{2}: & (\text { Assertion } \mathrm{U}(+)), \\ \text { or } & \\ U_{1} \geq U_{2}: & \text { (Assertion } \mathrm{U}(-)),\end{cases}
$$

with the internal energy increasing or internal energy decreasing condition applying to all adiabatic processes with the same values of the deformation variables.

From (4.12), along a line $\boldsymbol{X}^{\mathrm{D}}=\boldsymbol{X}_{0}^{\mathrm{D}}$ in $\Xi$ with either $\boldsymbol{X}=\left(\left(S, \boldsymbol{X}^{\mathrm{D}}\right)\right)$ or $\boldsymbol{X}=\left(\left(U, \boldsymbol{X}^{\mathrm{D}}\right)\right), \mathrm{d} U=T \mathrm{~d} S$. So $S$ and $U$ increase or decrease together if $T>0$ and one increases while the other decreases if $T<0$. In contrast to the assertion LY derived from the Lieb and Yngvason approach this 'standard' Carathéodory account allows both $S(+)$ and S(-) as possibilities arising from the application of Cth-I (Buchdahl, 1966, p. 76). It follows that the types of subsystems satisfy:

$$
\text { Cth-III }:=\left\{\begin{array}{c}
\mathrm{S}(+) \wedge\{[\mathrm{U}(+) \wedge \mathrm{T}(+)] \vee[\mathrm{U}(-) \wedge \mathrm{T}(-)]\} \\
\vee \\
\mathrm{S}(-) \wedge\{[\mathrm{U}(+) \wedge \mathrm{T}(-)] \vee[\mathrm{U}(-) \wedge \mathrm{T}(+)]\}
\end{array}\right\} .
$$

However, most authors (e.g. Buchdahl, op. cit. p. 77) follow Lieb and Yngvason in arguing for the truth of $U(+)$, that is that all systems are p-simple, in which case we have:

$$
\text { Cth-III+ := }\{\mathrm{U}(+) \wedge\{[S(+) \wedge \mathrm{T}(+)] \vee[S(-) \wedge \mathrm{T}(-)]\}
$$

It is clear that any one of LY, Cth-III and Cth-III+ implies Cth-I, since there will be, in any neighbourhood $\mathscr{N}_{\mathbf{x}}$ of $\boldsymbol{X} \in \Xi$, points, along the $X^{\mathrm{T}}$ axis above or below $\boldsymbol{X}$, inaccessible from $\boldsymbol{X}$ by an adiabatic transformation. The curves corresponding to Fig. 2(a) and (b) for $S(-)$ are shown in Fig. 5 (a) and (b) with the four curves combined in Fig. 6.

Fig. 3 could be taken to be a schematic representation of the case $S(-)$, where $\Xi:=\Xi^{(\mathrm{P})} \cup \Xi^{(\mathrm{N})}$ with the p-simple subsystem on the left having entropy decreasing upwards and $T<0$ and with the reverse for the n-simple subsystem on the right. In this case the dotted line $\boldsymbol{X}_{1}^{\prime}$ to $\boldsymbol{X}_{2}^{\prime}$ corresponds to the second half of the curve in Fig. 6.

\begin{tabular}{|c||c|c|}
\hline & $\mathrm{U}(+)$ & $\mathrm{U}(-)$ \\
\hline \hline $\mathrm{C}(+)$ & $\mathrm{T}(+) \wedge \mathrm{S}(+)$ & $\mathrm{T}(-) \wedge \mathrm{S}(+)$ \\
\hline $\mathrm{C}(-)$ & $\mathrm{T}(-) \wedge \mathrm{S}(-)$ & $\mathrm{T}(+) \wedge \mathrm{S}(-)$ \\
\hline
\end{tabular}




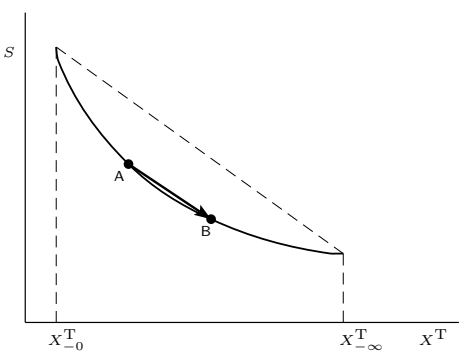

(a)

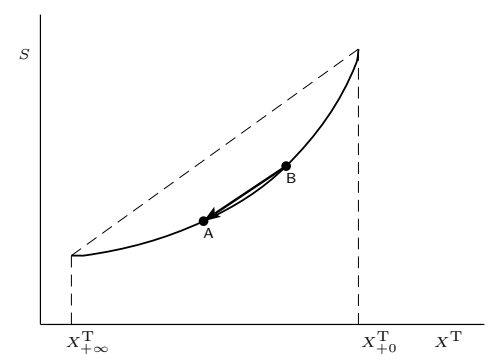

(b)

Figure 5: $S\left(\left(X^{\mathrm{T}}, \boldsymbol{X}^{\mathrm{D}}\right)\right)$ plotted against $X^{\mathrm{T}}$ for (a) an $\mathrm{n}$-simple system where $\mathrm{S}(-) \wedge \mathrm{U}(+) \wedge \mathrm{T}(-)$ applies and (b) a p-simple system where $\mathrm{S}(-) \wedge \mathrm{U}(-) \wedge \mathrm{T}(+)$ applies. The arrows denote possible adiabatic processes, $\mathrm{A}$ to $\mathrm{B}$ in (a) and $\mathrm{B}$ to $\mathrm{A}$ in (b) .

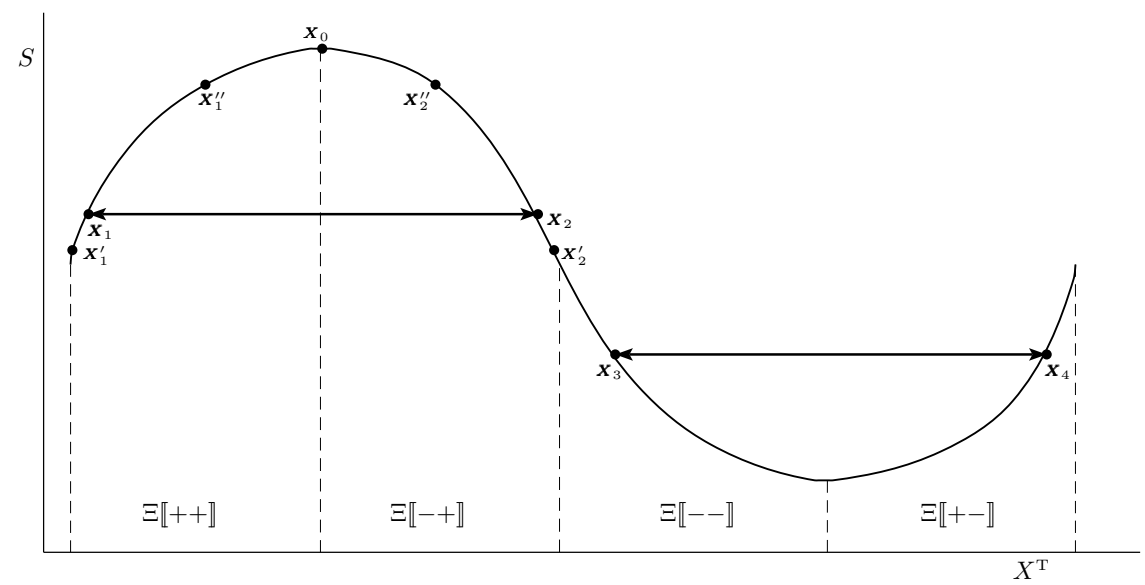

Figure 6: $S\left(\left(X^{\mathrm{T}}, \boldsymbol{X}^{\mathrm{D}}\right)\right)$ plotted against $X^{\mathrm{T}}$ for fixed $\boldsymbol{X}^{\mathrm{D}}$, with ranges for $\left.\left.\Xi \llbracket++\right], \Xi \llbracket-+\right], \Xi \llbracket+-\rrbracket$ and $\Xi \llbracket--\rrbracket$. The labelling of states on the first half of the curve is the same as in Fig. 3.

The types of system allowed by Cth-III can be identified by $\mathrm{U}( \pm) \wedge \mathrm{S}( \pm)$ for all choices of signs, but also by the sign of the temperature and whether the entropy is a concave or convex function, corresponding to $C( \pm)$, the heat capacity at constant deformation variables being respectively positive or negative. The equivalent ways of denoting the four types of systems are presented in Table 1, from which we see that we have a choice of pairs of signs to designate the type of a system. In fact for later use it turns out that the signs of $T( \pm) \wedge S( \pm)$ or exactly equivalently $T( \pm) \wedge C( \pm)$ (in that order) are most useful and systems or subsystems are identified as $\Xi[[ \pm \pm]$ with respect to these signs. The parts of the curve in Fig. 6 are labelled in that way. We have considered in detail the cases $\Xi:=\Xi[[++] \cup \Xi[-+]]$ and $\Xi:=\Xi[[--]] \cup \Xi[++-]$, which can both be represented by suitably interpreted versions of Fig. 3 . The form of Fig. 6 leads one to speculate on the possibility of a case $\Xi:=\Xi[[-+] \cup \Xi[--]$, where the two subspaces both have $T(-)$, but with $\mathrm{S}(+)$ and $\mathrm{S}(-)$ respectively. In fact a version of Fig. 3 reflected about the line $\mathrm{PQ}$, which now corresponds to $C(\boldsymbol{X})=0$ is consistent with that situation. The implications of all these subsystem divisions for the possibility of adiabatic processes across the $1 / T(\boldsymbol{X})=0$ or $C(\boldsymbol{X})=0$ boundary are discussed in Sect. 7.

\subsection{Summary of the Properties of Entropy}

Comparing the two approaches to the establishment of the entropy function $S(\boldsymbol{X})$ in Sects. 4.1 and 4.2 , we see that:

(i) Each establishes the scaling and additive properties of entropy. 
(ii) While the Lieb and Yngvason approach in Sect. 4.1 establishes that entropy is a non-decreasing function for all adiabatic processes, the approach described in Sect. 4.2 establishes that it is either a non-decreasing function or a non-increasing function.

Lieb and Yngvason use Axiom A-VII to show that entropy is a concave function on $\Xi$; specifically by applying $\mathrm{S}(+)$ to the special case $\boldsymbol{X}_{1}:=\left(\left(X_{1}^{\mathrm{T}}, \boldsymbol{X}^{\mathrm{D}}\right), \boldsymbol{X}_{2}:=\left(\left(X_{2}^{\mathrm{T}}, \boldsymbol{X}^{\mathrm{D}}\right)\right.\right.$ of Axiom A-VII

$$
\lambda S\left(\left(X_{1}^{\mathrm{T}}, \boldsymbol{X}^{\mathrm{D}}\right)\right)+(1-\lambda) S\left(\left(X_{2}^{\mathrm{T}}, \boldsymbol{X}^{\mathrm{D}}\right)\right) \leq S\left(\left(\lambda X_{1}^{\mathrm{T}}+(1-\lambda) X_{2}^{\mathrm{T}}, \boldsymbol{X}^{\mathrm{D}}\right)\right) .
$$

In fact (4.20) simply follows from $\mathrm{S}(+)$ and the homogeneity and extensivity of entropy (Prestipino and Giaquinta, 2003). The sign of temperature plays no part in the argument which equally well establishes concavity for Fig. 2 (a) or Fig. 2 (b). In the case where S(-) applies (4.20) is replaced by

$$
\lambda S\left(\left(X_{1}^{\mathrm{T}}, \boldsymbol{X}^{\mathrm{D}}\right)\right)+(1-\lambda) S\left(\left(X_{2}^{\mathrm{T}}, \boldsymbol{X}^{\mathrm{D}}\right)\right) \geq S\left(\left(\lambda X_{1}^{\mathrm{T}}+(1-\lambda) X_{2}^{\mathrm{T}}, \boldsymbol{X}^{\mathrm{D}}\right)\right),
$$

and the corresponding convex graphs of entropy against $X^{\mathrm{T}}$ are shown in Figs. 5 (a) and (b).

As we have already indicated in Sect. 3, it is a usually implicit assumption of thermodynamics that a process is possible between any two states of $\Xi$. In particular this applies to processes like $\mathrm{B}:=\left(\left(X^{\mathrm{T}}+\triangle X^{\mathrm{T}}, \boldsymbol{X}^{\mathrm{D}}\right)\right) \rightarrow\left(\left(X^{\mathrm{T}}, \boldsymbol{X}^{\mathrm{D}}\right)\right)=$ : $A$ or $A \rightarrow B$ between the labelled points on the curves shown in Figs. 2 (a), (b) and 5 (a), (b). In the first case concavity implies that

$$
S\left(\left(X^{\mathrm{T}}+\triangle X^{\mathrm{T}}, \boldsymbol{X}^{\mathrm{D}}\right)\right) \leq S\left(\left(X^{\mathrm{T}}, \boldsymbol{X}^{\mathrm{D}}\right)\right)+\triangle X^{\mathrm{T}}\left[T\left(\left(X^{\mathrm{T}}, \boldsymbol{X}^{\mathrm{D}}\right)\right)\right]^{-1},
$$

and in the second case convexity implies that

$$
S\left(\left(X^{\mathrm{T}}+\triangle X^{\mathrm{T}}, \boldsymbol{X}^{\mathrm{D}}\right)\right) \geq S\left(\left(X^{\mathrm{T}}, \boldsymbol{X}^{\mathrm{D}}\right)\right)+\triangle X^{\mathrm{T}}\left[T\left(\left(X^{\mathrm{T}}, \boldsymbol{X}^{\mathrm{D}}\right)\right)\right]^{-1} .
$$

From these inequalities the directions of the irrecoverable adiabatic processes $A \stackrel{A}{\rightarrow} B$ or $B \stackrel{A}{\rightarrow} A$, as shown in the figures, are evident.

\section{Thermal Equilibration and the Zeroth Law}

In our discussion of thermodynamics to this point we have been concerned with the properties of a single type of system in the whole or in a subspace of $\Xi$. In this and the following section we consider interactions between systems, and most particularly thermal interactions, where there is a flow of energy in the form of heat between two systems. We define processes in which two systems are joined to form a single system and in which a system is split into two subsystems. Lieb and Yngvason (1999, p. 55) formulated these notions in terms of two axioms, T-I and T-II asserting the existence of the operations of thermal joining and thermal splitting. We take a slightly different approach by defining the nature of the two processes before exploring whether or not they exist. Our more general approach is necessitated by the fact that, as already indicated, we are considering the four types of systems given by Cth-III and listed in Table 1 and for the moment we suppose that the two systems or subsystems involved in thermal splitting and joining are of the same type.

Thermal joining involves first putting two systems in thermal contact, meaning that energy can flow between them without change in their deformation variables. Initially the combined system is not necessarily in an equilibrium state; for this to come about a flow of energy in the form of heat needs to take place. This is called thermal equilibration. ${ }^{36}$ It is an adiabatic process and the final result is a system in a state described by a combination of the deformation variables of the two original systems and a thermal variable which is the sum of the corresponding thermal variables. Formally:

\footnotetext{
${ }^{36}$ It effectively invokes the minus first law of thermodynamics of Brown and Uffink (2001). They argue that none of the laws of thermodynamics actually asserts that a system not in equilibrium attains an equilibrium state and that it does so must constitute an addition law.
} 
Given two systems $\Xi_{\mathrm{A}}$ and $\Xi_{\mathrm{B}}$ with $\boldsymbol{X}_{\mathrm{A}}:=\left(\left(X_{\mathrm{A}}^{\mathrm{T}}, \boldsymbol{X}_{\mathrm{A}}^{\mathrm{D}}\right)\right) \in \Xi_{\mathrm{A}}$ and $\boldsymbol{X}_{\mathrm{B}}:=\left(\left(X_{\mathrm{B}}^{\mathrm{T}}, \boldsymbol{X}_{\mathrm{B}}^{\mathrm{D}}\right)\right) \in \Xi_{\mathrm{B}}$, the system $\Xi_{\mathrm{AB}}$ with $\boldsymbol{X}_{\mathrm{AB}}:=$ $\left(\left(X_{\mathrm{A}}^{\mathrm{T}}+X_{\mathrm{B}}^{\mathrm{T}}, \boldsymbol{X}_{\mathrm{A}}^{\mathrm{D}}, \boldsymbol{X}_{\mathrm{B}}^{\mathrm{D}}\right)\right) \in \Xi_{\mathrm{AB}}, \forall \boldsymbol{X}_{\mathrm{A}}$ and $\boldsymbol{X}_{\mathrm{B}}$ is called the thermal join of $\Xi_{\mathrm{A}}$ and $\Xi_{\mathrm{B}}$. It exists if $\left(\boldsymbol{X}_{\mathrm{A}}, \boldsymbol{X}_{\mathrm{B}}\right) \stackrel{\mathrm{A}}{\prec} \boldsymbol{X}_{\mathrm{AB}}, \forall\left(\boldsymbol{X}_{\mathrm{A}}, \boldsymbol{X}_{\mathrm{B}}\right) \in$ $\Xi_{\mathrm{A}} \times \Xi_{\mathrm{B}}$ and some $\boldsymbol{X}_{\mathrm{AB}} \in \Xi_{\mathrm{AB}}$. Any adiabatic process $\left(\boldsymbol{X}_{\mathrm{A}}, \boldsymbol{X}_{\mathrm{B}}\right) \stackrel{\mathrm{A}}{\rightarrow} \boldsymbol{X}_{\mathrm{AB}}$ is called a thermal joining with entropy satisfying

$$
\begin{array}{ll}
S_{\mathrm{A}}\left(\left(X_{\mathrm{A}}^{\mathrm{T}}, \boldsymbol{X}_{\mathrm{A}}^{\mathrm{D}}\right)+S_{\mathrm{B}}\left(X_{\mathrm{B}}^{\mathrm{T}}, \boldsymbol{X}_{\mathrm{B}}^{\mathrm{D}}\right)\right) \leq S_{\mathrm{AB}}\left(\left(X_{\mathrm{A}}^{\mathrm{T}}+X_{\mathrm{B}}^{\mathrm{T}}, \boldsymbol{X}_{\mathrm{A}}^{\mathrm{D}}, \boldsymbol{X}_{\mathrm{B}}^{\mathrm{D}}\right)\right), & \text { if } \mathrm{S}(+) \text { applies, } \\
\left.S_{\mathrm{A}}\left(\left(X_{\mathrm{A}}^{\mathrm{T}}, \boldsymbol{X}_{\mathrm{A}}^{\mathrm{D}}\right)+S_{\mathrm{B}}\left(X_{\mathrm{B}}^{\mathrm{T}}, \boldsymbol{X}_{\mathrm{B}}^{\mathrm{D}}\right)\right)\right) \geq S_{\mathrm{AB}}\left(\left(X_{\mathrm{A}}^{\mathrm{T}}+X_{\mathrm{B}}^{\mathrm{T}}, \boldsymbol{X}_{\mathrm{A}}^{\mathrm{D}}, \boldsymbol{X}_{\mathrm{B}}^{\mathrm{D}}\right)\right), & \text { if } \mathrm{S}(-) \text { applies. }
\end{array}
$$

The thermal splitting of a system into two systems depends first on some way of dividing the deformation variables into two subsets which will be the deformation variables of the resulting two systems. The obvious but not necessarily only way of identifying the members of the two subsets is if the system is a result of thermal joining. We shall assume that is true; in which case the process is essentially a reversal of thermal joining. Formally:

For the system $\Xi_{\mathrm{AB}}$ with $\boldsymbol{X}_{\mathrm{AB}}:=\left(\left(X_{\mathrm{AB}}^{\mathrm{T}}, \boldsymbol{X}_{\mathrm{A}}^{\mathrm{D}}, \boldsymbol{X}_{\mathrm{B}}^{\mathrm{D}}\right)\right) \in \Xi_{\mathrm{AB}}$, an adiabatic process $\boldsymbol{X}_{\mathrm{AB}} \stackrel{\mathrm{A}}{\rightarrow}\left(\boldsymbol{X}_{\mathrm{A}}, \boldsymbol{X}_{\mathrm{B}}\right) \in \Xi_{\mathrm{A}} \times \Xi_{\mathrm{B}}$, where $\left(\left(\tilde{X}_{\mathrm{A}}^{\mathrm{T}}, \boldsymbol{X}_{\mathrm{A}}^{\mathrm{D}}\right)\right) \in$ $\Xi_{\mathrm{A}},\left(\left(\tilde{X}_{\mathrm{B}}^{\mathrm{T}}, \boldsymbol{X}_{\mathrm{B}}^{\mathrm{D}}\right)\right) \in \Xi_{\mathrm{B}}$ and $\tilde{X}_{\mathrm{A}}^{\mathrm{T}}+\tilde{X}_{\mathrm{B}}^{\mathrm{T}}=X_{\mathrm{AB}}^{\mathrm{T}}$ is a thermal splitting of $\Xi_{\mathrm{AB}}$ iff $\boldsymbol{X}_{\mathrm{AB}} \stackrel{\mathrm{A}}{\prec}\left(\boldsymbol{X}_{\mathrm{A}}, \boldsymbol{X}_{\mathrm{B}}\right)$. Then

$$
S_{\mathrm{AB}}\left(\left(X_{\mathrm{AB}}^{\mathrm{T}}, \boldsymbol{X}_{\mathrm{A}}^{\mathrm{D}}, \boldsymbol{X}_{\mathrm{B}}^{\mathrm{D}}\right)\right)=S_{\mathrm{A}}\left(\left(\tilde{X}_{\mathrm{A}}^{\mathrm{T}}, \boldsymbol{X}_{\mathrm{A}}^{\mathrm{D}}\right)\right)+S_{\mathrm{B}}\left(\left(\tilde{X}_{\mathrm{B}}^{\mathrm{T}}, \boldsymbol{X}_{\mathrm{B}}^{\mathrm{D}}\right)\right), \quad \text { whether S }(+) \text { or S }(-) \text { applies. }
$$

Thermal joining and splitting can now be used to define thermal equilibrium. Thus:

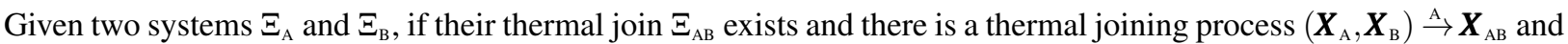
a thermal splitting process $\boldsymbol{X}_{\mathrm{AB}} \stackrel{\mathrm{A}}{\rightarrow}\left(\tilde{\boldsymbol{X}}_{\mathrm{A}}, \tilde{\boldsymbol{X}}_{\mathrm{B}}\right)$ such that $X_{\mathrm{A}}^{\mathrm{T}}=\tilde{X}_{\mathrm{A}}^{\mathrm{T}}$ and $X_{\mathrm{B}}^{\mathrm{T}}=\tilde{X}_{\mathrm{B}}^{\mathrm{T}}$ then $\Xi_{\mathrm{B}}$ and $\Xi_{\mathrm{B}}$ are in thermal equilibrium in states $\boldsymbol{X}_{\mathrm{A}}$ and $\boldsymbol{X}_{\mathrm{B}}$, respectively, denoted by $\boldsymbol{X}_{\mathrm{A}} \stackrel{\mathrm{T}}{\prec} \boldsymbol{X}_{\mathrm{B}}$.

The axioms T-I and T-II of Lieb and Yngvason simply assert the existence of a thermal join of two systems and of at least one thermal spitting of the resultingly joined system. They are, of course, referring to the splitting and joining of $\Xi[++]]$ systems, but there is no reason not to extend the assertion to all the other three cases where both systems are of the same type. We thus have a criterion for pairs of systems of each of these four types being in equilibrium. Fundamental to this is (Buchdahl, 1966, p. 29): ${ }^{37}$

The Zeroth Law of Thermodynamics: The thermal equilibrium relationship $\succ^{\mathrm{T}}$ is transitive.

Suppose that the systems $\Xi_{\mathrm{A}}$ and $\Xi_{\mathrm{B}}$ respectively in the states $\left(\left(X^{\mathrm{T}}-\triangle X^{\mathrm{T}}, \boldsymbol{X}_{\mathrm{A}}^{\mathrm{D}}\right)\right)$ and $\left(\left(\triangle X^{\mathrm{T}}, \boldsymbol{X}_{\mathrm{B}}^{\mathrm{D}}\right)\right)$ are in thermal equilibrium. This means that they can be thermally joined and then thermally split (however many times we like) without changing their states. For this to happen equalities must apply in the thermal joining equations (5.1); meaning that equilibrium is given when $\triangle X^{\mathrm{T}}$ maximises in the case of a $\mathrm{S}(+)$ systems and minimises in the case of a $\mathrm{S}(-)$ systems ${ }^{38}$

$$
S_{\mathrm{AB}}\left(\left(X^{\mathrm{T}}, \boldsymbol{X}_{\mathrm{A}}^{\mathrm{D}}, \boldsymbol{X}_{\mathrm{B}}^{\mathrm{D}} ; \triangle X^{\mathrm{T}}\right)\right):=S_{\mathrm{A}}\left(\left(X^{\mathrm{T}}-\triangle X^{\mathrm{T}}, \boldsymbol{X}_{\mathrm{A}}^{\mathrm{D}}\right)\right)+S_{\mathrm{B}}\left(\left(\triangle X^{\mathrm{T}}, \boldsymbol{X}_{\mathrm{B}}^{\mathrm{D}}\right)\right)
$$

at fixed $X^{\mathrm{T}}, \boldsymbol{X}_{\mathrm{A}}^{\mathrm{D}}$ and $\boldsymbol{X}_{\mathrm{B}}^{\mathrm{D}}$. From (2.1) this extremum condition is

$$
T_{\mathrm{A}}\left(\left(X^{\mathrm{T}}-\triangle X^{\mathrm{T}}, \boldsymbol{X}_{\mathrm{A}}^{\mathrm{D}}\right)\right)=T_{\mathrm{B}}\left(\left(\triangle X^{\mathrm{T}}, \boldsymbol{X}_{\mathrm{B}}^{\mathrm{D}}\right)\right) .
$$

On the other hand if equality of temperature is taken as the criterion for thermal equilibrium, then, from (4.22) and (4.23), $\Xi_{\mathrm{A}}$ in state $\left(\left(X_{\mathrm{A}}^{\mathrm{T}}, \boldsymbol{X}_{\mathrm{A}}^{\mathrm{D}}\right)\right)$ and $\Xi_{\mathrm{B}}$ in state $\left(\left(X_{\mathrm{B}}^{\mathrm{T}}, \boldsymbol{X}_{\mathrm{B}}^{\mathrm{D}}\right)\right)$ are in thermal equilibrium if

$$
\begin{array}{ll}
S_{\mathrm{A}}\left(\left(X_{\mathrm{A}}^{\mathrm{T}}-\triangle X^{\mathrm{T}}, \boldsymbol{X}_{\mathrm{A}}^{\mathrm{D}}\right)\right)+S_{\mathrm{B}}\left(\left(X_{\mathrm{B}}^{\mathrm{T}}+\triangle X^{\mathrm{T}}, \boldsymbol{X}_{\mathrm{B}}^{\mathrm{D}}\right)\right) \leq S_{\mathrm{A}}\left(\left(X_{\mathrm{A}}^{\mathrm{T}}, \boldsymbol{X}_{\mathrm{A}}^{\mathrm{D}}\right)\right)+S_{\mathrm{B}}\left(\left(X_{\mathrm{B}}^{\mathrm{T}}, \boldsymbol{X}_{\mathrm{B}}^{\mathrm{D}}\right)\right), & \forall \triangle X^{\mathrm{T}}, \quad \text { if } \mathrm{S}(+) \text { applies }, \\
S_{\mathrm{A}}\left(\left(X_{\mathrm{A}}^{\mathrm{T}}-\triangle X^{\mathrm{T}}, \boldsymbol{X}_{\mathrm{A}}^{\mathrm{D}}\right)\right)+S_{\mathrm{B}}\left(\left(X_{\mathrm{B}}^{\mathrm{T}}+\triangle X^{\mathrm{T}}, \boldsymbol{X}_{\mathrm{B}}^{\mathrm{D}}\right)\right) \geq S_{\mathrm{A}}\left(\left(X_{\mathrm{A}}^{\mathrm{T}}, \boldsymbol{X}_{\mathrm{A}}^{\mathrm{D}}\right)\right)+S_{\mathrm{B}}\left(\left(X_{\mathrm{B}}^{\mathrm{T}}, \boldsymbol{X}_{\mathrm{B}}^{\mathrm{D}}\right)\right), & \forall \triangle X^{\mathrm{T}}, \quad \text { if } \mathrm{S}(-) \text { applies },
\end{array}
$$

\footnotetext{
${ }^{37}$ Lieb and Yngvason assert the zeroth law as their axiom T-III prior to the establishment of the equal temperatures condition (5.4). As pointed out by Hilbert et al. (2014), the transitivity of the zeroth law can be captured only if the systems in the description of thermal equilibrium are understood both in an actual and potential sense. Thus we may describe the situation 'if two systems are thermally joined' or 'if two systems were to be thermally joined'.

${ }^{38}$ This result is proved more formally, for $\mathrm{U}(+) \wedge \mathrm{S}(+)$ systems, by Lieb and Yngvason (op. cit., p. 57), before their introduction of temperature.
} 
which is equivalent to the extremum condition (5.3). At the end of a process of thermal equilibration $\Xi_{\mathrm{A}}$ and $\Xi_{\mathrm{B}}$ are at the same temperature, or equivalently pairs of systems of the same type are in thermal equilibrium if they are at the same temperature.

We now consider the possibility of two systems of different types being in equilibrium. Before confining their attention to $\Xi[[++]]$ systems Lieb and Yngvason (op. cit., p. 56) argued that a $\Xi[[++]]$ system and a $\Xi[[-+]]$ system could not be in thermal equilibrium and we can see that is indeed the case. Since, although the entropy argument contained in (5.3) and (5.4) applies, the two systems can never have equal temperatures one being negative and the other positive. The same argument would apply to a pair consisting of a $\Xi[+-]]$ system and a $\Xi[--]]$ system. It is clear that the entropy argument breaks down for $\mathrm{S}(+), \mathrm{S}(-)$ pairs of systems and this absence of the possibility of equilibrium is reinforced in the case where the temperatures have differences of signs. However, it is of interest to note the case of system pairs $\Xi[[++]], \Xi[[+-]]$ and $\Xi[[--]], \Xi[-+]]$. There all the arguments concerning equilibration and extrema of entropy break down, but the two systems could have the same temperature. Of course, a state of thermal contact in this case would be highly unstable. Setting aside this speculation we can adopt the language used by Lieb and Yngvason (op. cit., p. 47, and cited above) where

A Thermodynamic World is a maximal set of thermodynamic systems related by the possibility of thermal equilibrium.

Then we see that thermodynamic systems or the subspaces of systems fall into the four worlds as given in Table 1 and distinguished most conveniently by the pairs of signs of their temperature and heat capacity. Most accounts of thermodynamics restrict attention to positive temperatures. The whole point of the current work is, however, to explore the possibility of negative temperatures.This question is distinct from the question of the sign of the heat capacity with respect to constant deformation variables. Most physical systems exhibit a positive heat capacity ${ }^{39}$ and, although in Sect. 6 we include all types of systems, our discussions of statistical mechanics in Parts II and III concentrates solely on $\Xi[[++]]$ and $\Xi[[-+]]$ systems or subsystems.

\section{Comparing Versions of the Second Law}

It is standard in the literature (e.g. Pippard, 1961, pp. 29,30) to present three versions of the second law, that due to Carathéodory, the first and second versions of which, denoted as Cth-I and Cth-II, we gave in Sect. 3, and those due to Kelvin and Planck, and Clausius. In its modern expression (Buchdahl, 1966, p. 89) the Kelvin-Planck version of the second law takes the form: ${ }^{40}$

$\mathrm{K}-\mathrm{P}$ : It is impossible to construct an engine which, operating in a cycle, will produce no effect other than the extraction of heat from a reservoir and the performance of an equivalent of work.

In its modern expression (Buchdahl, op. cit.) the Clausius version of the second law takes the form: ${ }^{41}$

\footnotetext{
${ }^{39}$ There are some systems for which the heat capacity is negative (Lynden-Bell, 1999). These are inhomogeneous systems, including gravitating objects like as stars and galaxies. According to the virial theorem, for a self-gravitating body like a star or an interstellar gas cloud, the average potential energy $U_{\mathrm{PE}}$ and the average kinetic energy $U_{\mathrm{KE}}$ are locked together in the relation $U_{\mathrm{PE}}=-2 U_{\mathrm{KE}}$. The total energy $U=U_{\mathrm{PE}}+U_{\mathrm{KE}}$ therefore obeys $U=-U_{\mathrm{KE}}$. If the system loses energy by, for example, radiating energy into space, then $U_{\mathrm{KE}}$ increases. Given that the temperature is defined by $U_{\mathrm{KE}}$ the system can be said to have a negative heat capacity.

${ }^{40}$ The original wording of Kelvin (Thomson, 1882, p. 179) is that: "It is impossible, by means of inanimate material agency, to derive mechanical effect from any portion of matter by cooling it below the temperature of the coldest of the surrounding objects." Planck (1903) considered a number of versions of the second law. The most apposite in the present context is in his Sect. 116: "It is impossible to construct an engine which will work in a complete cycle, and produce no effect except the raising of a weight and the cooling of a heat-reservoir." Another, from his Sect. 133 is quoted in a footnote on page 44

${ }^{41}$ The wording given by Clausius (1865) is that: "Heat can never pass from a colder to a warmer body without some other change, connected therewith, occurring at the same time."
} 
$\mathrm{Cl}$ : It is impossible to construct a device which, operating in a cycle, will produce no effect other than the transfer of heat from a cooler to a hotter body.

At this point, and in view of later discussion, it is worth pausing to explore what is mean by a 'reservoir'. In fact in the literature (Huang 1963, p. 5; Emch and Liu 2002, p. 338) the terms 'heat bath', 'reservoir' and 'heat reservoir' are used as synonyms and taken to mean "a system so large that the gain or loss of any finite amount of heat does not change its temperature" (Huang, op. cit.) or "systems so large that their properties cannot be observably affected by letting much smaller systems be brought in contact with them" (Emch and Liu, op. cit.). However, as observed by Lavis (2018), the 'largeness' which appears here is, from a theoretical point of view, not essential. ${ }^{42}$ What is required is that energy in the form of heat can be drawn from the system without changing it temperature. And this can be achieved by:

An isothermal reservoir $\Xi_{R}$, which is "a simple system whose temperature $T_{R}$ remains constant by virtue of its entropy being a linear function of its thermal variable $X_{\mathrm{R}}^{\mathrm{T}}$ and such that, in any process, its deformation variable $\boldsymbol{X}_{\mathrm{R}}^{\mathrm{D}}$ also remains constant" (Lavis, op. cit.).

The implication of this is that the deformation variable $\boldsymbol{X}_{\mathrm{R}}^{\mathrm{D}}$ can effectively be ignored. The sole variable determining the state of the system is the thermal variable $X_{\mathrm{R}}^{\mathrm{T}}$, but the temperature $T_{\mathrm{R}}$ enters, not as a variable but as a parameter intrinsic to the definition of $\Xi_{R}$. Thus for an isothermal reservoir $\Xi_{R}$ we modify our previous notation by designating its state and nature by $\left(\left(X_{R}^{\mathrm{T}} ; T_{\mathrm{R}}\right)\right)$. The only changes in internal energy of the reservoir are the result of a flow of heat; (4.12) in this case simply relates $S(\boldsymbol{X})$ and $U(\boldsymbol{X})$, becoming for a process $\boldsymbol{X}_{\mathrm{R}} \rightarrow \boldsymbol{X}_{\mathrm{R}}^{\prime}$

$$
T\left[S\left(\boldsymbol{X}_{\mathrm{R}}\right)-S\left(\boldsymbol{X}_{\mathrm{R}}^{\prime}\right)\right]=U\left(\boldsymbol{X}_{\mathrm{R}}\right)-U\left(\boldsymbol{X}_{\mathrm{R}}^{\prime}\right)=Q,
$$

where $Q$ is the heat that flows from the reservoir.

An important role played by isothermal reservoirs is to effect an isothermal process $\boldsymbol{X} \stackrel{\mathrm{I}}{\rightarrow} \boldsymbol{X}^{\prime}$ in a system $\Xi$. That is to say, a process where $T(\boldsymbol{X})=T\left(\boldsymbol{X}^{\prime}\right)$. Suppose that $\Xi$ in state $\boldsymbol{X}:=\left(\left(X^{\mathrm{T}}, \boldsymbol{X}^{\mathrm{D}}\right)\right)$ and $\Xi_{\mathrm{R}}$ in state $\boldsymbol{X}_{\mathrm{R}}:=\left(\left(X_{\mathrm{R}}^{\mathrm{T}} ; T_{\mathrm{R}}\right)\right)$ are thermally joined to form the system $\Xi_{\mathrm{CR}}$ which equilibrates into state $\boldsymbol{X}_{\mathrm{CR}}:=\left(\left(X^{\mathrm{T}}+X_{\mathrm{R}}^{\mathrm{T}}, \boldsymbol{X}^{\mathrm{D}} ; T_{\mathrm{R}}\right)\right)$. The joined system performs an adiabatic process in which $\boldsymbol{X}^{\mathrm{D}} \rightarrow \boldsymbol{X}^{\mathrm{D}}$ and work $W:=W^{(\mathrm{A})}\left(\boldsymbol{X}_{\mathrm{CR}}, \boldsymbol{X}_{\mathrm{CR}}^{\prime}\right)$ is performed on the environment. The system is then split leaving $\Xi$ in state $\boldsymbol{X}^{\prime}:=\left(\left(X^{\mathrm{T} \prime}, \boldsymbol{X}^{\mathrm{D}}\right)\right)$ and $\Xi_{\mathrm{R}}$ in state $\boldsymbol{X}_{\mathrm{R}}^{\prime}:=\left(\left(X_{\mathrm{R}}^{\mathrm{T}} ; T_{\mathrm{R}}\right)\right)$. Given that the work $W$ must have been performed by $\Xi$, the changes in internal energy for $\Xi_{\mathrm{R}}$ and $\Xi$ are

$$
\triangle^{(\mathrm{R})} U:=X_{\mathrm{R}}^{\mathrm{T} \prime}-X_{\mathrm{R}}^{\mathrm{T}}=-Q=T_{\mathrm{R}} \triangle^{(\mathrm{R})} S, \quad \triangle U:=X^{\mathrm{T} \prime}-X^{\mathrm{T}}=Q-W,
$$

where $\triangle^{(\mathrm{R})} S:=S\left(\boldsymbol{X}_{\mathrm{R}}^{\prime}\right)-S\left(\boldsymbol{X}_{\mathrm{R}}\right)$ and $Q$ is the heat which flows from $\Xi_{\mathrm{R}}$ to $\Xi$.

There are many discussions in the literature concerning the equivalence (or otherwise) of $\mathrm{K}-\mathrm{P}, \mathrm{Cl}$ and $\mathrm{Cth}-\mathrm{I}$. In this section we shall restrict attention to a more limited task; to determine whether $\mathrm{K}-\mathrm{P}$ and $\mathrm{Cl}$ can be derived in the four cases given by Cth-III in (4.18). To this end we consider a cycle of processes between four points $\boldsymbol{X}_{\mathrm{A}}, \boldsymbol{X}_{\mathrm{B}}, \boldsymbol{X}_{\mathrm{C}}, \boldsymbol{X}_{\mathrm{D}}, \in \Xi$, with

$$
T\left(\boldsymbol{X}_{\mathrm{A}}\right)=T\left(\boldsymbol{X}_{\mathrm{B}}\right), \quad S\left(\boldsymbol{X}_{\mathrm{B}}\right)=S\left(\boldsymbol{X}_{\mathrm{C}}\right), \quad T\left(\boldsymbol{X}_{\mathrm{C}}\right)=T\left(\boldsymbol{X}_{\mathrm{D}}\right), \quad S\left(\boldsymbol{X}_{\mathrm{D}}\right)=S\left(\boldsymbol{X}_{\mathrm{A}}\right) .
$$

If $\Xi$ is of a single thermodynamic type, it follows from (4.16) that adiabatic processes $\boldsymbol{X}_{\mathrm{B}} \stackrel{\mathrm{A}}{\rightarrow} \boldsymbol{X}_{\mathrm{C}}$ and $\boldsymbol{X}_{\mathrm{D}} \stackrel{\mathrm{A}}{\rightarrow} \boldsymbol{X}_{\mathrm{A}}$ are

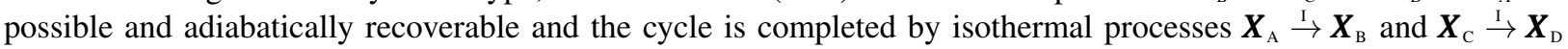
effected by thermally coupling the system to isothermal reservoirs $\Xi_{\mathrm{R} 1}$ and $\Xi_{\mathrm{R} 2}$, both of the same thermodynamic type as $\Xi$, and performing on the combined systems adiabatic processes which are not necessarily recoverable. (Although, of course, the isothermal processes for $\Xi$ are recoverable.) We also include the possibility that the two reservoirs are of different thermodynamic types, specifically that they have different temperature signs but with the same direction

\footnotetext{
${ }^{42}$ Except in so far as it avoids an upper bound on the heat that can be drawn from the reservoir (see Sect. 12.1).
} 
of entropy flow in an adiabatic process. For such a cycle to be possible and for the adiabatic processes $\boldsymbol{X}_{\mathrm{B}} \stackrel{\mathrm{A}}{\rightarrow} \boldsymbol{X}_{\mathrm{C}}$ and $\boldsymbol{X}_{\mathrm{D}} \stackrel{\mathrm{A}}{\rightarrow} \boldsymbol{X}_{\mathrm{A}}$ to be, even in principle, realizable $\Xi$ must be the union of subspaces of the types of the two reservoirs as represented in Fig. 3. As already indicated, the question of adiabatic processes between two subspaces of a system with opposite temperature signs, is considered in more depth in Sect. 7.

It is worth noting at this point that in many discussions of this cycle (see e.g. Pippard, 1961, p. 33; Huang, 1963, p. 11) it is assumed that the system undergoes equilibrium processes (suitably interpreted) along curves between the four points $\boldsymbol{X}_{\mathrm{A}}, \boldsymbol{X}_{\mathrm{B}}, \boldsymbol{X}_{\mathrm{C}}, \boldsymbol{X}_{\mathrm{D}}$. This assumption is unnecessary for our analysis; the only thing defining the geometry of the cycle is its four corners.

The temperatures of $\Xi_{\mathrm{R} 1}$ and $\Xi_{\mathrm{R} 2}$ are respectively $T_{\mathrm{R} 1}=T\left(\boldsymbol{X}_{\mathrm{A}}\right)=T\left(\boldsymbol{X}_{\mathrm{B}}\right)$ and $T_{\mathrm{R} 2}=T\left(\boldsymbol{X}_{\mathrm{C}}\right)=T\left(\boldsymbol{X}_{\mathrm{D}}\right)$ with

$$
\frac{1}{T_{\mathrm{R} 2}}>\frac{1}{T_{\mathrm{R} 1}}
$$

For all cycles, $\Xi_{R 1}$ and $\Xi_{R 2}$ with the smaller and larger values of inverse temperature, are referred to respectively as the hot and cold reservoirs, so that a reservoir at a negative temperature is always hotter than a reservoir at a positive temperature, and:

I: For $\boldsymbol{X}_{\mathrm{A}} \stackrel{\mathrm{I}}{\rightarrow} \boldsymbol{X}_{\mathrm{B}}, \Xi$ is thermally joined to $\Xi_{\mathrm{R} 1}$ at temperature $T_{\mathrm{R} 1}$. From (6.2) the changes in internal energy for $\Xi_{\mathrm{R} 1}$ and $\Xi$ are

$$
\triangle_{\mathrm{AB}}^{(\mathrm{R})} U:=X_{\mathrm{RB}}^{\mathrm{T}}-X_{\mathrm{RA}}^{\mathrm{T}}=-Q_{\mathrm{AB}}=T_{\mathrm{R} 1} \triangle_{\mathrm{AB}}^{(\mathrm{R})} S, \quad \triangle_{\mathrm{AB}} U:=X_{\mathrm{B}}^{\mathrm{T}}-X_{\mathrm{A}}^{\mathrm{T}}=Q_{\mathrm{AB}}-W_{\mathrm{AB}},
$$

where $\triangle_{\mathrm{AB}}^{(\mathrm{R})} S:=S\left(\boldsymbol{X}_{\mathrm{RB}}\right)-S\left(\boldsymbol{X}_{\mathrm{RA}}\right)$ and $Q_{\mathrm{AB}}$ is the heat which flows from $\Xi_{\mathrm{R} 1}$ to $\Xi$.

II: For $\boldsymbol{X}_{\mathrm{B}} \stackrel{\mathrm{A}}{\rightarrow} \boldsymbol{X}_{\mathrm{C}}$, from (4.7), the change in internal energy is

$$
\triangle_{\mathrm{BC}} U=-W^{(\mathrm{A})}\left(\boldsymbol{X}_{\mathrm{B}}, \boldsymbol{X}_{\mathrm{C}}\right)=:-W_{\mathrm{BC}} .
$$

III: For $\boldsymbol{X}_{\mathrm{C}} \stackrel{\mathrm{I}}{\rightarrow} \boldsymbol{X}_{\mathrm{D}}, \Xi$ is thermally joined to $\Xi_{\mathrm{R} 2}$ at temperature $T_{\mathrm{R} 2}$. From (6.2),

$$
\triangle_{\mathrm{CD}}^{(\mathrm{R})} U=-Q_{\mathrm{CD}}=T_{\mathrm{R} 2} \triangle_{\mathrm{CD}}^{(\mathrm{R})} S, \quad \triangle_{\mathrm{CD}} U=Q_{\mathrm{CD}}-W_{\mathrm{CD}} .
$$

IV: For $\boldsymbol{X}_{\mathrm{D}} \stackrel{\mathrm{A}}{\rightarrow} \boldsymbol{X}_{\mathrm{A}}$, from (4.7),

$$
\triangle_{\mathrm{DA}} U=-W^{(\mathrm{A})}\left(\boldsymbol{X}_{\mathrm{D}}, \boldsymbol{X}_{\mathrm{A}}\right)=:-W_{\mathrm{DA}} .
$$

Since the internal energy of $\Xi$ returns to its initial state

$$
W_{\mathrm{T}}:=W_{\mathrm{AB}}+W_{\mathrm{BC}}+W_{\mathrm{CD}}+W_{\mathrm{DA}}=Q_{\mathrm{AB}}+Q_{\mathrm{CD}}
$$

The total work performed on the environment is equal to the total heat absorbed from the reservoirs.

As already indicated the most convenient designation for a system is as one of $T( \pm) \wedge S( \pm)$. If both the reservoirs $\Xi_{R 1}$ and $\Xi_{\mathrm{R} 2}$ and the system $\Xi$ have the same sign for $S( \pm)$ then, for entropy, there are two cases to consider:

(i) $\mathrm{S}(+)$ applies to all adiabatic processes and in particular, for the processes I and III where $\Xi$ is thermally joined to the reservoirs,

$$
\frac{Q_{\mathrm{AB}}}{T_{\mathrm{R} 1}}=-\triangle_{\mathrm{AB}}^{(\mathrm{R})} S \leq \triangle S \leq \triangle_{\mathrm{CD}}^{(\mathrm{R})} S=-\frac{Q_{\mathrm{CD}}}{T_{\mathrm{R} 2}},
$$

where

$$
\triangle S:=S\left(\boldsymbol{X}_{\mathrm{B}}\right)-S\left(\boldsymbol{X}_{\mathrm{A}}\right)=S\left(\boldsymbol{X}_{\mathrm{C}}\right)-S\left(\boldsymbol{X}_{\mathrm{D}}\right) .
$$




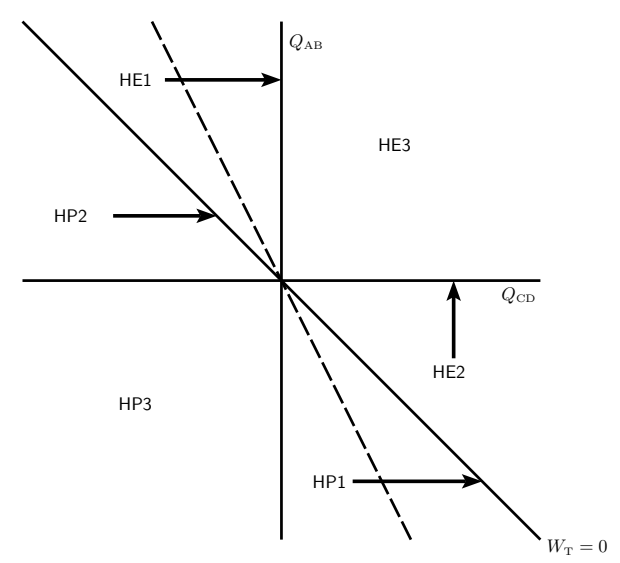

Figure 7: The regions for each of the cases of heat engines and pumps for $\mathrm{T}(+)\left(T_{\mathrm{R} 1}>T_{\mathrm{R} 2}>0\right)$. Arrows point to the 'ideal' situation, where no heat is wasted in the heat engine and no work is done driving the heat pump. The broken line, given by (6.22), separates the region when $\mathrm{S}(+)$ applies on the left from that where $\mathrm{S}(-)$ applies on the right.

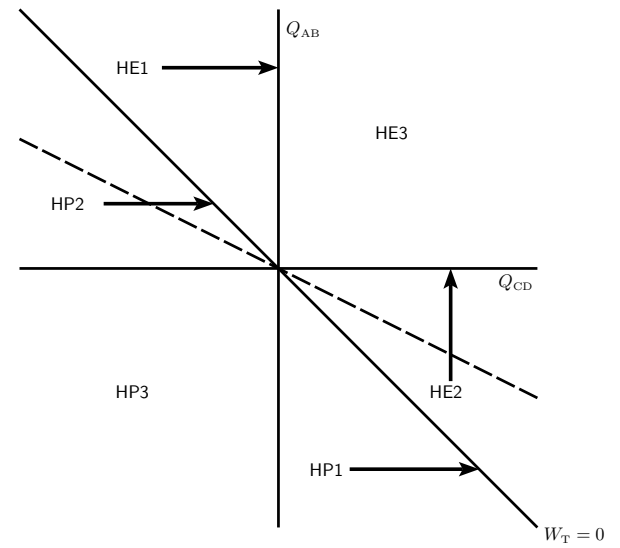

Figure 8: The regions for each of the cases of heat engines and pumps for $\mathrm{T}(-)\left(0>T_{\mathrm{R} 1}>T_{\mathrm{R} 2}\right)$. Arrows point to the 'ideal' situation, where no heat is wasted in the heat engine and no work is done driving the heat pump. The broken line, given by (6.22), separates the region when $\mathrm{S}(+)$ applies on the right from that where $\mathrm{S}(-)$ applies on the left.

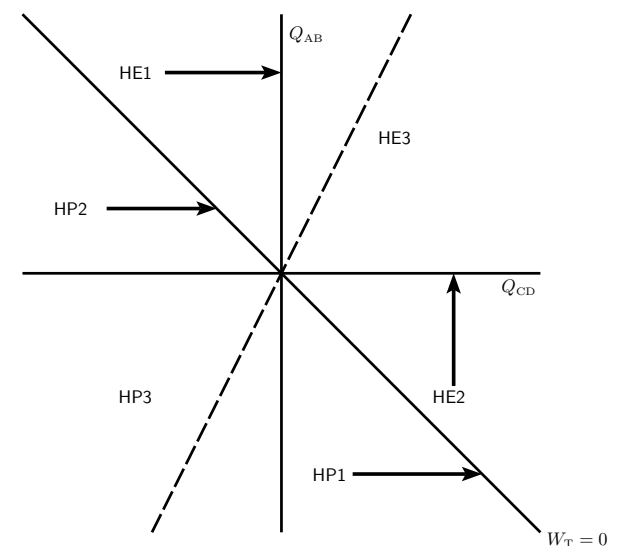

Figure 9: The regions for each of the cases of heat engines and pumps for $\mathrm{T}( \pm)\left(T_{\mathrm{R} 2}>0>T_{\mathrm{R} 1}\right)$. Arrows point to the 'ideal' situation, where no heat is wasted in the heat engine and no work is done driving the heat pump. The broken line, given by (6.22), separates the region when $\mathrm{S}(+)$ applies on the left from that where $\mathrm{S}(-)$ applies on the right 
(ii) $\mathrm{S}(-)$ applies to all adiabatic processes and in particular, for the processes I and III where $\Xi$ is thermally joined to the reservoirs,

$$
\frac{Q_{\mathrm{AB}}}{T_{\mathrm{R} 1}}=-\triangle_{\mathrm{AB}}^{(\mathrm{R})} S \geq \triangle S \geq \triangle_{\mathrm{CD}}^{(\mathrm{R})} S=-\frac{Q_{\mathrm{CD}}}{T_{\mathrm{R} 2}}
$$

And for temperature we consider three cases:

(a) $\mathrm{T}(+)$ applies to $\Xi_{\mathrm{R} 1}, \Xi_{\mathrm{R} 2}$ and $\Xi$; and

$$
T_{\mathrm{R} 1}>T_{\mathrm{R} 2}>0 \quad \Longrightarrow \quad 0<\frac{T_{\mathrm{R} 2}}{T_{\mathrm{R} 1}}<1 .
$$

(b) $\mathrm{T}(-)$ applies to $\Xi_{\mathrm{R} 1}, \Xi_{\mathrm{R} 2}$ and $\Xi$; and

$$
0>T_{\mathrm{R} 1}>T_{\mathrm{R} 2} \Longrightarrow \frac{T_{\mathrm{R} 2}}{T_{\mathrm{R} 1}}>1 .
$$

(c) $\mathrm{T}(+)$ applies to $\Xi_{\mathrm{R} 2}$ and $\mathrm{T}(-)$ applies to $\Xi_{\mathrm{R} 1}$. This case, which we denote by $\mathrm{T}( \pm)$, implies that

$$
\Xi:=\Xi[[++] \cup \Xi[[-+] \text { or } \Xi:=\Xi[[+-]] \cup \Xi[--]] \text {, }
$$

with

$$
T_{\mathrm{R} 2}>0>T_{\mathrm{R} 1} \Longrightarrow \frac{T_{\mathrm{R} 2}}{T_{\mathrm{R} 1}}<0 .
$$

For the total work $W_{\mathrm{T}}$ there are three possibilities:

- When $W_{\mathrm{T}} \geq 0$ the cycle is acting as a heat engine; heat is extracted from one or both of the reservoirs and work is performed on the environment. The limiting cases are the ideal engine, where all the heat is converted into work and none is 'wasted' by being drained into the other reservoir and the worst engine, where all the heat is wasted and no work is done. K-P refers to the situation where heat flows from either the hot reservoir or the cold reservoir and the cycle is prevented from being ideal by either inequality (6.10) or inequality (6.12).

- When $W_{\mathrm{T}} \leq 0$ the cycle is acting as a heat pump (or refrigerator); work is performed on the system by pumping heat from one reservoir to the other or into both reservoirs. The limiting cases are the ideal pump, where all the heat passes from one reservoir to the other without work being needed and the worst pump, where work is done but no heat passes from one reservoir to the other. $\mathrm{Cl}$ refers to the situation where the heat is pumped from the cold reservoir and is prevented from being ideal by either inequality (6.10) or inequality (6.12).

- When $W_{\mathrm{T}}=0$ the cycle can be regarded as acting both as a heat engine and a heat pump; heat passes from one reservoir to the other with no work being done on or by the environment. It is therefore, as already indicated, the worst possible heat engine and an ideal heat pump. As we shall see this symmetry relationship between the worst possible heat engine and the ideal heat pump is an example of the wider symmetry between heat engines and heat pumps.

Combining the two entropy cases (i) and (ii) with the three temperature cases (a), (b) and (c) there are six possibilities for the heat flow: three where the cycle operates as a heat engine:

HE1: $Q_{\mathrm{AB}}>0, Q_{\mathrm{AB}} \geq-Q_{\mathrm{CD}} \geq 0$ : heat $Q_{\mathrm{AB}}$ is extracted from the hot reservoir $\Xi_{\mathrm{R} 1}$ and $-Q_{\mathrm{CD}}$ is the waste energy supplied to the cold reservoir $\Xi_{\mathrm{R} 2}$.. The efficiency of the cycle is

$$
\eta:=\frac{W_{\mathrm{T}}}{Q_{\mathrm{AB}}}=1-\frac{\left|Q_{\mathrm{CD}}\right|}{Q_{\mathrm{AB}}},
$$


where $0 \leq \eta \leq 1$, with the ideal engine $Q_{\mathrm{CD}}=0$ and worst engine $Q_{\mathrm{CD}}=-Q_{\mathrm{AB}}$ corresponding to $\eta=1$ and $\eta=0$, respectively. However, (6.10) and (6.12) impose the condition

$$
\begin{array}{ll}
\frac{T_{\mathrm{R} 2}}{T_{\mathrm{R} 1}} \leq \frac{\left|Q_{\mathrm{CD}}\right|}{Q_{\mathrm{AB}}} \leq 1 \quad \text { for cases } \quad[\mathrm{T}(+) \vee \mathrm{T}( \pm)] \wedge \mathrm{S}(+) \text { and } \quad \mathrm{T}(-) \wedge \mathrm{S}(-), \\
\frac{T_{\mathrm{R} 2}}{T_{\mathrm{R} 1}} \geq \frac{\left|Q_{\mathrm{CD}}\right|}{Q_{\mathrm{AB}}} \geq 0 \quad \text { for cases } \quad[\mathrm{T}(+) \vee \mathrm{T}( \pm)] \wedge \mathrm{S}(-) \text { and } \quad \mathrm{T}(-) \wedge \mathrm{S}(+) .
\end{array}
$$

From (6.14), if $\mathrm{T}(-)$, the inequalities (6.18) are impossible to satisfy and the inequalities (6.19) are always satisfied. On the other hand, from (6.16), if $\mathrm{T}( \pm)$, the inequalities (6.19) are impossible to satisfy and the inequalities (6.18) are always satisfied. So cycles satisfying $\mathrm{T}(-) \wedge \mathrm{S}(-)$ and $\mathrm{T}( \pm) \wedge \mathrm{S}(-)$ are not possible (NP) and for cycles satisfying $\mathrm{T}(-) \wedge \mathrm{S}(+)$ and $\mathrm{T}( \pm) \wedge \mathrm{S}(+)$ the efficiency is unconstrained (UC) and can range over the whole interval $[0,1]$. There remain the two cases where $\mathrm{T}(+)$ is satisfied:

(1) From (6.13) and (6.18) when $\mathrm{T}(+) \wedge \mathrm{S}(+)$

$$
0 \leq \eta \leq \eta^{(\mathrm{c})}:=1-\frac{T_{\mathrm{R} 2}}{T_{\mathrm{T} 1}}<1
$$

The cycle is unconstrained from acting in a worst way but constrained from acting in an ideal way (CFI) as a heat engine. From (6.3) the adiabatic processes $\boldsymbol{X}_{\mathrm{B}} \stackrel{\mathrm{A}}{\rightarrow} \boldsymbol{X}_{\mathrm{C}}$ and $\boldsymbol{X}_{\mathrm{D}} \stackrel{\mathrm{A}}{\rightarrow} \boldsymbol{X}_{\mathrm{A}}$ are recoverable and if the inequalities in (6.10) become equalities, the adiabatic processes where $\Xi$ is thermally joined to the reservoirs are also recoverable. Then the whole cycle is recoverable and is called a Carnot cycle ${ }^{43}$ with Carnot efficiency $\eta^{(c)}$, the maximum efficiency at which the engine can perform.

(2) From (6.13) and (6.19) when $\mathrm{T}(+) \wedge \mathrm{S}(-)$

$$
0<\eta^{(c)} \leq \eta \leq 1
$$

The cycle is unconstrained from acting in an ideal way but constrained from acting in a worst way (CFW) as a heat engine. The Carnot efficiency is now the least efficiency.

In passing we note that in the two completely unconstrained cases $\mathrm{T}(-) \wedge \mathrm{S}(+)$ and $\mathrm{T}( \pm) \wedge \mathrm{S}(+), \eta^{(\mathrm{c})}<0$ and $\eta^{(\mathrm{c})}>1$, respectively.

HE2: $Q_{\mathrm{CD}}>0,0 \geq Q_{\mathrm{AB}} \geq-Q_{\mathrm{CD}}$ : heat $Q_{\mathrm{CD}}$ is extracted from the cold reservoir $\Xi_{\mathrm{R} 2}$ and $-Q_{\mathrm{AB}}$ is waste energy supplied to the hot reservoir $\Xi_{R 1}$.

HE3: $Q_{\mathrm{AB}}>0$ and $Q_{\mathrm{CD}}>0$; heat is extracted from both reservoirs and work is performed on the environment.

And three cases where it acts as a heat pump:

HP1: $Q_{\mathrm{AB}}<0,0 \leq Q_{\mathrm{CD}} \leq-Q_{\mathrm{AB}}$; work is performed by the environment extracting heat $Q_{\mathrm{CD}}$ from the cold reservoir $\Xi_{\mathrm{R} 2}$ and inputting heat $-Q_{\mathrm{AB}}$ into the hot reservoir $\Xi_{\mathrm{R} 1}$.

HP2: $Q_{\mathrm{CD}}<0,0 \leq Q_{\mathrm{AB}} \leq-Q_{\mathrm{CD}}$; work is performed by the environment extracting heat $Q_{\mathrm{AB}}$ from the hot reservoir $\Xi_{\mathrm{R} 1}$ and inputting heat $-Q_{\mathrm{CD}}$ into the cold reservoir $\Xi_{\mathrm{R} 2}$.

HP3: $Q_{\mathrm{AB}}<0$, and $Q_{\mathrm{CD}}<0$; work is performed by the environment and heat absorbed by both reservoirs.

\footnotetext{
${ }^{43}$ Sometimes (see, for exmple, Landsberg, 1961, Sect. 25) this cycle of processes is called a 'Carnot cycle' irrespective of whether it is recoverable.
} 
The detailed analysis given above for the various cases of a heat engine HE1 can be applied in a similar way to the other two cases of a heat engine and to the three cases of a heat pump. The regions for each of the cases of engines and pumps are shown in Figs. 7, 8 and 9, for $\mathrm{T}(+), \mathrm{T}(-)$ and $\mathrm{T}( \pm)$, with, in each figure, the region of engines separated from the region of pumps by the line $W_{\mathrm{T}}=0\left(Q_{\mathrm{AB}}=-Q_{\mathrm{CD}}\right)$ and regions for $\mathrm{S}(+)$ and $\mathrm{S}(-)$ separated by the broken line given by

$$
\frac{Q_{\mathrm{AB}}}{T_{\mathrm{R} 1}}=-\frac{Q_{\mathrm{CD}}}{T_{\mathrm{R} 2}}, \text { that is } \eta=\eta^{(\mathrm{c})} .
$$

The information in the figures is transcribed into Table 2. For HE3 and HP3, where heat is provided from both reservoirs and to both reservoirs respectively, the cycle is either possible (P) or not possible (NP) because the conditions (6.4) and (6.10) or (6.12) cannot be satisfied. All the other cases divide like HE1 into cases where the cycles are not possible (NP) and, where possible into those which are unconstrained (UC) by (6.10) or (6.12) and those that are. If a constraint applies to a heat engine or pump it may be constrained from operating in an ideal way (CFI) or in its worst way $(\mathrm{CFW})$, but not both. An engine or pump is prevented from acting in a ideal way by conditions (6.10) or (6.12) being violated beyond a boundary represented by the broken lines in Figs. 7 and 8 . These correspond to the best recoverable (Carnot) operation of the cycle. The symmetry inherent in the inequalities (6.10) and (6.12) are manifested in Table 2 in that:

- The four $3 \times 3$ blocks map into each other in a clockwise or an anticlockwise direction by interchanging the first two rows and first two columns, with the interchanges $\mathrm{CFI} \leftrightarrow \mathrm{CFW}$ when a heat pump interchanges with a heat engine.

- Changing the sign of temperatures interchanges hot and cold reservoirs. It thus gives interchanges HE1 $\leftrightarrow \mathrm{HE} 2$ and HP1 $\leftrightarrow \mathrm{HP} 2$, and between the inequalities (6.10) and (6.12), mapping between top and bottom blocks for both engines and pumps.

Table 2 and Figs. 7 - 9 can now be used to examine validity conditions for $\mathrm{K}-\mathrm{P}$ and $\mathrm{Cl}$.

K-P makes no reference to the relative temperatures of the two reservoirs. It simply asserts that a heat engine extracting heat from one reservoir and doing work cannot perform in an ideal way, that is to say without any other effect. It therefore countenances the case HE1, where heat is extracted from the hot reservoir, and HE2, where heat is extracted from the cold reservoir. The case of HE3 is interesting. No heat is being wasted and this performance of the cycle could in the spirit of the law be regarded as highly desirable (ideal) and therefore a violation of K-P. However, on the obvious literal reading it satisfies the law (if it is possible) because the heat extracted from the second reservoir, whichever it is, is another 'effect', and this is how we choose to treat it.

$\mathrm{Cl}$ is in some ways simpler. It asserts the impossibility of ideal heat flow, that is to say without any work being expended, in a specific direction, from a cold reservoir to a hot reservoir. It applies therefore only to case HP1 and makes no reference either way to cases HP2 and HP3 although it is interesting no note that HP2 predicts the flow of heat from the hot to the cold reservoir, without the expenditure of work, both when the temperature of each is positive (which is otherwise obvious) and both is negative, and when the hot reservoir is at a negative temperature and the cold reservoir at a positive temperature.

Reading from Table 2 the condition for the failure of either law is UC or CFW. Both in their original formulations and in the modifications proposed by Ramsey (1956) and Landsberg (1977), the law of increasing entropy $\mathrm{S}(+)$ is assumed. First restricting attention to that case we see that $\mathrm{Cl}$ does not fail for any of the signs of the temperatures and $\mathrm{K}-\mathrm{P}$ fails if heat is emitted from only one reservoir and that reservoir is at a negative temperature.

For Kelvin at the time of formulating his version of the second law the version due to Clausius was well-known and he observes that "It is easily shown that, although this [Clausius' version of the second law] and the axiom I have used [his version of the second law] are different in form, either is a consequence of the other" (Thomson, 1882, p. 180).

On the other hand it is worth examining in some detail the comments of Ehrenfest-Afanassjewa (1925, p. 938). ${ }^{44}$ She first states her temperature axiom (Axiom D) that:

\footnotetext{
${ }^{44}$ I am indebted to Roman Frigg for this English translation.
} 
"The absolute temperature has the same sign for all thermodynamic states."45

She then makes the following observations:

(i) "Without this condition one could carry out a Carnot cycle that contains an isothermal process of positive absolute temperature and an isothermal process of negative absolute temperature. It would then be possible to retrieve heat from both reservoirs, and [as a consequence] no heat would flow from a higher temperature to a lower temperature in the conversion of heat into work, as the principle of Clausius requires."

This remark, refers to the case $\mathrm{T}( \pm) \wedge \mathrm{S}(+)$, with heat flowing from both reservoirs and being converted into work. This is, therefore, the heat engine case HE3, which Ehrenfest-Afanassjewa correctly takes as possible. However, it is not relevant to $\mathrm{Cl}$ which is concerned solely with heat pumps, where work is converted into heat and not the other way round, and for reasons we have described above it is also not a violation of $\mathrm{K}-\mathrm{P}$.

(ii) "That the absolute temperature has an unalterable sign is assumed to be obvious. From the above we now see how essential this property is for Clausius' principle. By contrast, Axiom D is not necessary for Thomson's [Kelvin's] principle, which only says that one has to use more than one heat reservoir to transform heat into work by dint of a cyclical process."

Contrary to this observation we have shown that Axiom $D(\mathrm{~T}(+) \wedge \mathrm{S}(+))$ is necessary for $\mathrm{K}-\mathrm{P}$, since otherwise the cycle can work in an ideal way emitting heat from a reservoir at a negative temperature and turning it all into work, whether or not the second reservoir is at a negative temperature.

(iii) "We see that the two principles do not say the same thing."

We have shown this to be true, but not for the reasons advanced by Ehrenfest-Afanassjewa.

(iv) "From the existence of the entropy alone neither Thomson's nor Clausius' principle can be deduced."

If the burden of this remark is that the signs of the temperatures of the reservoirs are also needed then it is true for Thomson's principle (K-P) but not for Clausius' principle $(\mathrm{Cl})$ which is independent of the signs of the temperatures.

In a similar vein Cooper (1967) speculates that "it is theoretically conceivable that a perpetual machine of the second kind could be universally impossible [that is to say K-P will always hold] but that Clausius' law does not hold for negative temperatures." However, our analysis and that of Ramsey (1956) and Landsberg (1977) has shown that, when the condition $\mathrm{S}(+)$ applies in all adiabatic processes, the Clausius law continues to be valid and the Kelvin-Planck version fails. The modification of the K-P proposed by Ramsey is: ${ }^{46}$

K-P/R: It is impossible to construct an engine which will operate in a closed cycle and provide no effect other than (i) the extraction of heat from a positive-temperature reservoir with the performance of an equivalent amount of work, or (ii) the rejection of heat into a negative-temperature reservoir with the corresponding work being done on the engine.

This identifies in (i) the impossible cases $\mathrm{T}(+) \wedge \mathrm{S}(+)$, HE1, HE2 and HE3; and in (ii) the impossible case $\mathrm{T}( \pm) \wedge \mathrm{S}(+)$, HE2. It also identifies in (i) $\mathrm{T}( \pm) \wedge \mathrm{S}(+)$, HE3 and in (ii) $\mathrm{T}(-) \wedge \mathrm{S}(+)$, HE3, which although they are possible cycles, are, as indicated above, excluded by the law since there is more that one effect.

The version due to Landsberg is:

\footnotetext{
${ }^{45}$ The German 'Wärmezustand' literally means 'heat state'; the term denotes a state characterised in terms of heat.

${ }^{46}$ The 'on' in the last line would appear to be a slip of the pen since the point of a heat engine is that the work is done by it on the environment.
} 


\begin{tabular}{c|ccc||c|cc}
\hline & HE1 & HE2 & HE3 & HP1 & HP2 & HP3 \\
\hline \hline $\mathrm{T}(+) \wedge \mathrm{S}(+)$ & CFI & NP & NP & CFI & UC & $P$ \\
\hline $\mathrm{T}(-) \wedge \mathrm{S}(+)$ & UC & CFW & $\mathbf{P}$ & NP & $C F W$ & $N P$ \\
\hline $\mathrm{T}( \pm) \wedge \mathrm{S}(+)$ & UC & NP & $\mathbf{P}$ & NP & $U C$ & $P$ \\
\hline \hline $\mathrm{T}(+) \wedge \mathrm{S}(-)$ & CFW & UC & $\mathbf{P}$ & CFW & $N P$ & $N P$ \\
\hline $\mathrm{T}(-) \wedge \mathrm{S}(-)$ & NP & CFI & NP & UC & $C F I$ & $P$ \\
\hline $\mathrm{T}( \pm) \wedge \mathrm{S}(-)$ & NP & UC & $\mathbf{P}$ & UC & $N P$ & $P$ \\
\hline
\end{tabular}

Table 2: Cycle behaviour for all cases of heat engines and heat pumps. For HE3 and HP3 the cycles are either possible or not possible (P or NP). For HE1, HP1, HE2 and HP2 the cycles are either not possible (NP) or unconstrained (UC) or constrained from ideal behaviour (CFI) or worse behaviour (CFW). Cases where the appropriate form of the second law is satisfied are shown in bold font. The irrelevant cases are shown in slanting font.

$\mathrm{K}-\mathrm{P} / \mathrm{L}$ : Heat can be completely converted into work by a heat engine which takes a medium through a cyclic process, if and only if, that heat is withdrawn from a negative temperature reservoir.

This identifies the three possible cases $\mathrm{T}(-) \wedge \mathrm{S}(+), \mathrm{HE} 1$ and $\mathrm{HE} 2$, and $\mathrm{T}( \pm) \wedge \mathrm{S}(+), \mathrm{HE} 1$. It could also be understood, unlike $\mathrm{K}-\mathrm{P} / \mathrm{R}$ as including the cases $\mathrm{T}( \pm) \wedge \mathrm{S}(+)$ and $\mathrm{T}(-) \wedge \mathrm{S}(+), \mathrm{HE}$.

Landsberg et al. (1980) give a proof that these two versions are equivalent, but as we have seen it is obvious from our approach.

Of course, as we have already indicated, both these new version of the Kelvin-Planck law assume $\mathrm{S}(+)$, the law of non-decreasing entropy, as do both the original Kelvin-Planck and Clausius versions. From Table 2, the modification to the latter when both $\mathrm{S}(+)$ and $\mathrm{S}(-)$ are allowed is straightforward:

$\mathrm{Cl}^{\star}$ : It is impossible to construct a device which, operating in a cycle, will produce no effect other than transfer of heat between two bodies of different temperatures if and only if (i) the heat flow is from the cooler to the hotter body and entropy is a non-decreasing quantity in all adiabatic processes, or (ii) the heat flow is from the hotter to the cooler body and entropy is a non-increasing quantity in all adiabatic processes.

In the case of the Kelvin-Planck law in this most general situation it is simplest to modify the Landsberg version:

$\mathrm{K}-\mathrm{P} / \mathrm{L}^{\star}$ : Heat can be completely converted into work by a heat engine which takes a medium through a cyclic process if and only if (i) that heat is withdrawn from a negative temperature reservoir and entropy is a non-decreasing quantity in all adiabatic processes, or (ii) that heat is withdrawn from a positive temperature reservoir and entropy is a nonincreasing quantity in all adiabatic processes.

\section{Processes Between Positive and Negative Temperatures}

The $\mathrm{T}( \pm)$ cycle in Sect. 6 was predicated on a minimum decomposition $\Xi[+++] \cup \Xi[[-+]$ or $\Xi[[+-] \cup \Xi[[--]]$ of the cycling system $\Xi$ and of there being a recoverable adiabatic process between states in the two subspaces of the decomposition. In this section we consider the possibilities for such processes. As we made clear at the beginning of Sect. 3, it is assumed that there is always some way to effect a process between two states of $\Xi$ and this applies to the case of processes between states in different subspaces of the decomposition. The important question is the existence and properties of an adiabatic process of this kind. 
This is on the one hand a practical question. According to the first law, ${ }^{\mathrm{A}} \prec \boldsymbol{X}^{\prime}$ if there exists a way, within the limitations discussed in Sect. 3, for work to be performed by the system on its environment to implement a process $\boldsymbol{X} \stackrel{\mathrm{A}}{\rightarrow} \boldsymbol{X}^{\prime}$. We have in Sect. 1.1, presented the experimental evidence for a physical system being caused to perform a process from a state of positive temperature to one of negative temperature. The thermodynamic question of whether the process is performed adiabatically and more specifically whether it occurs between states of equal entropy is not one addressed by the experimenters. However, in the case of the experiments on LiF crystals by Pound (1951) and Ramsey and Pound (1951), where the process from positive to negative temperature is achieved by rapidly reversing nuclear spins using a magnetic field it would seem plausible that the process is adiabatic.

On the other hand, the question has theoretical content. Does an adiabatic process between different subspaces, and in particular subspaces with different signs of the temperature, violate any principle of thermodynamics? The statements $\mathrm{U}( \pm)$ and $\mathrm{S}( \pm)$ with a particular choice of signs both apply to adiabatic processes within one subspace. But it is clear that both cannot apply with the same signs to processes between different subspaces. Thus an adiabatic process between states in $\Xi[[++]$ and $\Xi[[-+]$ is between subspaces within which $S(+)$ is satisfied but with $U(+)$ and $\mathrm{U}(-)$ respectively. This would seem to imply that the condition $\mathrm{S}(+)$ survives for adiabatic processes between $\Xi[++]]$ and $\Xi[[-+]$. However, we begin with a rather weaker assertion, which covers the cases of the recoverable adiabatic processes. $\boldsymbol{X}_{\mathrm{B}} \stackrel{\mathrm{A}}{\rightarrow} \boldsymbol{X}_{\mathrm{C}}$ and $\boldsymbol{X}_{\mathrm{D}} \stackrel{\mathrm{A}}{\rightarrow} \boldsymbol{X}_{\mathrm{A}}$ for the $\mathrm{T}( \pm)$ cycle in Sect. 6.

For $\Xi[[++] \cup \Xi[-+]]$ as represented in Fig. 3, and using the same state labelling as in that figure and Fig. 6, we propose the following:

Adiabatic Hypothesis: If $\boldsymbol{X}_{1} \in \Xi[[++]]$ and $\boldsymbol{X}_{2} \in \Xi[[-+]]$ and $S\left(\boldsymbol{X}_{1}\right)=S\left(\boldsymbol{X}_{2}\right)$ then $\boldsymbol{X}_{1} \succ^{\mathrm{A}} \prec \boldsymbol{X}_{2}$.

From which is is simple to show that:

Theorem 7.1. Given that both $\Xi[[++]]$ and $\Xi\left[[-+]\right.$ have a continuous range of entropy $\left[S_{\mathrm{L}}, S_{\mathrm{U}}\right]$ and $\boldsymbol{X}_{1}^{\prime} \in \Xi[[++]]$ and $\boldsymbol{X}_{2}^{\prime \prime} \in \Xi\left[[-+]\right.$ then $S\left(\boldsymbol{X}_{1}^{\prime}\right) \leq S\left(\boldsymbol{X}_{2}^{\prime \prime}\right) \Longrightarrow \boldsymbol{X}_{1}^{\prime} \stackrel{\text { A }}{\prec} \boldsymbol{X}_{2}^{\prime \prime}$.

The same argument can, of course, be applied to establish $\boldsymbol{X}_{2}^{\prime} \stackrel{\text { A }}{\prec} \boldsymbol{X}_{1}^{\prime \prime}$ and, with decreasing rather than increasing entropy, to establish adiabatic processes between $\Xi[--]$ and $\Xi[[+-]$. The reasoning is, however, inapplicable to the possibility of adiabatic processes between two subspaces with one where $S(+)$ applies and one where $S(-)$ applies. In the case illustrated in Fig. 6 the adiabatic assertion cannot be applied because there is no overlap in the ranges of entropy. However, even if this were not the case the most that could be assumed is the existence of adiabatic processes between states of equal entropy and nothing between states with unequal entropies. Although, as we showed in Sect. 4.2, the division $\Xi[-+] \cup \Xi[[--]]$ does not conflict with any principles of thermodynamics, the existence and nature of adiabatic processes between the subspaces is an open question. From the experimental point of view evidence suggests the existence of systems with negative heat capacity (see the footnote on page 18). But we are unaware of any evidence for systems having subsystems with opposite signs for the heat capacity, let alone adiabatic processes between states in these two subspaces.

We now examine the validity of our Adiabatic Hypothesis concentrating for simplicity on adiabatic processes between subspaces $\Xi[[++]]$ and $\Xi[-+]]$ since this is the situation for the $T( \pm)$ cycle in Sect. 6 which involves two adiabatically recoverable processes $\boldsymbol{X}_{\mathrm{B}} \stackrel{\mathrm{A}}{\rightarrow} \boldsymbol{X}_{\mathrm{C}}$ and $\boldsymbol{X}_{\mathrm{D}} \stackrel{\mathrm{A}}{\rightarrow} \boldsymbol{X}_{\mathrm{A}}$, where $\boldsymbol{X}_{\mathrm{A}}, \boldsymbol{X}_{\mathrm{B}} \in \Xi[[-+]]$ and $\boldsymbol{X}_{\mathrm{C}}, \boldsymbol{X}_{\mathrm{D}} \in \Xi[[++]]$. For the $\mathrm{T}(+)$ or $\mathrm{T}(-)$ cycle adiabatic recoverability implies $S\left(\boldsymbol{X}_{\mathrm{B}}\right)=S\left(\boldsymbol{X}_{\mathrm{C}}\right)$ and $S\left(\boldsymbol{X}_{\mathrm{D}}\right)=S\left(\boldsymbol{X}_{\mathrm{A}}\right)$ with each pair of points lying on an adiabat. However, Ramsey (1956, p. 22) remarks that "no means has yet been devised by which a Carnot cycle can be operated between a positive and a negative temperature" and although he does not detail the way that the cycle is performed the generally accepted picture is made explicit by Pippard (1961, p. 52) who asserts that "no isentropic [adiabatic] surfaces connect positive and negative temperatures, and therefore no reversible [recoverable] cycle may be constructed." These quotes from Ramsey and Pippard expose the crux of our discussion, since they, along with many other authors (e.g. Huang, 1963; Tremblay, 1976; Tykodi, 1978) suppose that the cycle, traces out equilibrium isothermal and adiabatic processes in $\Xi$. If this were the case then the equilibrium processes $\boldsymbol{X}_{\mathrm{B}} \stackrel{\mathrm{A}}{\rightarrow} \boldsymbol{X}_{\mathrm{C}}$ and $\boldsymbol{X}_{\mathrm{D}} \stackrel{\mathrm{A}}{\rightarrow} \boldsymbol{X}_{\mathrm{A}}$ would have to pass through points where the temperature changed sign and as we have seen such points have $\beta:=1 / T=0$. For a system (like $\mathfrak{B}_{m}$, with finite $m$, but not necessarily with only one deformation variable) which has a finite number of energy levels, Tremblay (1976) has proved that entropy has exactly one value on the surface $\beta=0$ and that $\exists \varepsilon>0$ such that this value is greater than that on any hypersurfaces where $\beta \in(-\varepsilon, 0) \cup(0, \varepsilon)$. For this class of models 
Tremblay correctly infers that this substantiates the first part of the remark by Pippard given above, that no adiabat can connect the two states with equal entropy in the two subspaces. But this we have already shown in Sect. 4.1, since it would follow in the case from Lieb and Yngvason's Thm. 3.3 that the decomposition $\Xi[[++] \cup \Xi[[=+]]$ would be impossible in that case. So the problem arises only if adiabats are needed for the processes $\boldsymbol{X}_{\mathrm{B}} \stackrel{\mathrm{A}}{\rightarrow} \boldsymbol{X}_{\mathrm{C}}$ and $\boldsymbol{X}_{\mathrm{D}} \stackrel{\mathrm{A}}{\rightarrow} \boldsymbol{X}_{\mathrm{A}}$, and in the description of the cycles in Sect. 6 we have shown that the only states which have a role are $\boldsymbol{X}_{\mathrm{A}}, \boldsymbol{X}_{\mathrm{B}}, \boldsymbol{X}_{\mathrm{C}}$ and $\boldsymbol{X}_{\mathrm{D}}$; not any kind of equilibrium processes (however interpreted) between them. To reemphasis the point; we know that some process $\boldsymbol{X}_{\mathrm{B}} \rightarrow \boldsymbol{X}_{\mathrm{C}}$ is possible, but can it be performed adiabatically? The most we can say in answer to this question is (a) that the kind of sudden change of state envisaged in the cycles in Sect. 6 and allowed by our Adiabatic Hypothesis is more in tune with the experiments described in Sect. 1.1 than is some continuous change of state. And (b) that the argument advanced by the authors cited above based on the non-existence of adiabats does not exclude the situation we are describing here and does not provide an argument for excluding adiabatic processes between states with a different temperature sign, as in the cases represented by Fig. 9 and lines 3 and 6 of Table 2 .

\section{Part II}

\section{Statistical Mechanical Considerations}

As indicated in the title the main interest of this paper is in the possibility that the temperature of a system can be either positive or negative. In treating a cycle of processes in Sect. 6, we included the case where a system undergoes an adiabatic process between states with different signs for the temperature. In the statistical mechanical discussion in this part we concentrate solely on the temperatures of single states. We shall also restrict attention to systems where $\mathrm{S}(+)$ applies that is the cases $\mathrm{T}(+) \wedge \mathrm{S}(+)$ and $\mathrm{T}(-) \wedge \mathrm{S}(+)$; meaning, from Table 1, that the heat capacity at constant deformation variables is positive. Entropy is a concave function of energy, non-decreasing in adiabatic processes.

\section{Systems and Environments}

Suppose that, as in Part I, the system is simple; that is to say, there is one thermal variable identified as the internal energy $U$. We shall also, for simplicity, suppose that there is one deformation variable $X,{ }^{47}$ when (4.12) becomes

$$
T \mathrm{~d} S=\mathrm{d} U-\xi \mathrm{d} X
$$

or equivalently

$$
T \mathrm{~d} S=\mathrm{d} H+X \mathrm{~d} \xi, \quad \text { where } \quad H:=U-\xi X,
$$

is the enthalpy.

In statistical mechanics the modelling of the system in different physical conditions is by way of different distributions over the underlying microstates mediated through the Hamiltonian. In each case a thermodynamical potential (or free energy) is determined as a function of the independent controllable variables with the associate dependent variables and response functions being determined as derivatives of the potential. A dependent extensive variable is also given as the expectation value of its corresponding statistical mechanical variable with respect to the appropriate distribution. The expectation value of the Hamiltonian is the internal energy $U$ for a mechanically isolated system and the enthalpy $H$ for a system in mechanical interaction with its environment. The value of the Hamiltonian, which we denote by $E$ and refer to as the mechanical energy of the system, is taken to be bounded below by $E_{\min }$ and above by $E_{\max }$. Apart from in the discussion of an isothermal reservoir in Sect. 11.3 the existence of a lower bound is not normally problematic, and the existence of an upper bound and its role in the occurrence of negative temperatures has already been explored in Sect. 1.

For the simple system considered here there are four environments according as the system is, on the one hand, either thermally isolated (TI) or in thermal contact with its environment (TC) in the form of an isothermal reservoir,

\footnotetext{
${ }^{47}$ This is in addition to the size parameter $\boldsymbol{\aleph}$ introduced in Sect. 9. The generalization to a larger set of deformation variables is straightforward.
} 


\begin{tabular}{|c||c|c|c|c|}
\hline & TI-MI & TI-MC & TC-MI & TC-MC \\
\hline \hline Control Variables & $U, X$ & $H, \xi$ & $T, X$ & $T, \xi$ \\
\hline Thermodynamic Potential & Entropy & Entropy & $\begin{array}{c}\text { Helmholtz Free Energy } \\
A:=U-S T\end{array}$ & $\begin{array}{c}\text { Gibbs Free Energy } \\
G:=H-S T\end{array}$ \\
\hline \multirow{3}{*}{ Differential Relationships } & $\frac{1}{T}=\frac{\partial S}{\partial U}$ & $\frac{1}{T}=\frac{\partial S}{\partial H}$ & $S=-\frac{\partial A}{\partial T}$ & $S=-\frac{\partial G}{\partial T}$ \\
& $\frac{\xi}{T}=-\frac{\partial S}{\partial X}$ & $\frac{X}{T}=\frac{\partial S}{\partial \xi}$ & $\xi=\frac{\partial A}{\partial X}$ & $X=-\frac{\partial G}{\partial \xi}$ \\
\hline Heat Capacity & $C_{X}:=\left(\frac{\partial T}{\partial U}\right)^{-1}$ & $C_{\xi}:=\left(\frac{\partial T}{\partial H}\right)^{-1}$ & $C_{X}:=T \frac{\partial S}{\partial T}$ & $C_{\xi}:=T \frac{\partial S}{\partial T}$ \\
or $C_{\xi}$ at Constant $\xi$ & & & \\
\hline
\end{tabular}

Table 3: Summary of thermodynamic environments. In each case a partial derivative with respect to one independent variable implies that the other is kept constant.

and, on the other hand, mechanically isolated (MI), so that $X$ is fixed, or in mechanical contact with its environment (MC), so that it is subject to manipulation of $\xi$. Table 3 summarizes the relevant thermodynamic properties for each of these four combinations. For a TI system temperature is positive or negative according as entropy is a monotonically increasing or decreasing function of $U$ for a MI system, or $H$ for a MC system, as shown in Figs. 2(a), 5(b) or 2(b), 5(a), respectively. Heat capacity is positive or negative according as entropy is concave or convex as a function of $U$ or $H$ as in Figs. 2(a),(b) or 5(a),(b) respectively. ${ }^{48}$ It is, of course, the case that any of these behaviours could be exhibited by a system over different ranges of $U$ or $H$.

The statistical mechanical distributions for TI-MI and TC-MI systems are called, respectively the microcanonical distribution and canonical distribution and for a TC-MC system the Gibbs distribution. There does not appear to be a generally accepted name for the distribution of a TI-MC system and we shall call it the micromechanical distribution.

\section{Small, Large and Infinite Systems: The Equivalence of Distributions}

For a TC system the temperature is a controllable variable whose value $T$ is determined by contact with the environment, which is an isothermal reservoir. The internal energy $U$ is identified with the expectation value of $E$, with the fluctuations of $E / U$ satisfying (Lavis, 2015, p. 15) a fluctuation-response function relationship which, for a TC-MI system, takes the form

$$
\sqrt{\operatorname{Var}\left[\frac{E}{U}\right]}=\frac{\sqrt{\left\langle E^{2} \mid T, X\right\rangle-[\langle E \mid T, X\rangle]^{2}}}{\langle E \mid T, X\rangle}=\frac{T \sqrt{C_{X}}}{U}
$$

where as already indicated we have restricted attention to the case where the heat capacity $C_{X}>0$. Similar results apply for a TC-MC system and also for fluctuations in $\widehat{X}$, the statistical mechanical variable associated with $X$.

The size of a system, measured by a size parameter $\aleph$, is an important property in statistical mechanics. In principle any extensive deformation variable can play this role, but the important point is that once chosen it is treated as a parameter rather than a variable. In both the case of the $\mathfrak{B}_{m}$ model introduced in Sect. 10.2 and the perfect fluid of Appen. $\mathrm{D}, \boldsymbol{N}:=N$, the number of particles in the system. ${ }^{49}$ From (9.1) it can be seen that the magnitude of the fluctuations of $E / U$ measured in terms of their standard deviation is of order $1 / \sqrt{\aleph}$ meaning that the fluctuations decrease with the size of the system. ${ }^{50}$ Since fluctuations of uncontrolled extensive variables are

\footnotetext{
${ }^{48}$ Although, as we have already indicated, we shall restrict attention to the positive case.

${ }^{49}$ Although, in the latter case, the presentation could be reformulated so that the volume $V$ played that role.

${ }^{50}$ This statement must be qualified in the case of the neighbourhood of a phase transition. If such a transition occurs at a temperature $T_{\mathrm{C}}$ then in the thermodynamic limit, $C_{X} \propto\left(T-T_{\mathrm{C}}\right)^{-\alpha}$, where typically $\alpha$ has a small positive value, and for large but finite systems this singular behaviour is approached in the form of steep maxima in $C_{X}$. This means that the closer one is to a phase transition the larger $\boldsymbol{\aleph}$ needs to be to suppress fluctuations, with the relationship between size and fluctuations failing at the transition. Henceforth we shall, for the sake of simplicity, suppose that our discussion is predicated on the assumption that we are not near any phase transitions.
} 
characteristic of statistical mechanics and not present in equilibrium thermodynamics, it is only in the thermodynamic limit $\mathfrak{N} \rightarrow \infty$ that statistical mechanics reduces to thermodynamics ${ }^{51}$ and one might expect that in this limit results derived from different distributions become equal. The question of the conditions under which this happens has attracted much attention over the years (see, for example, Ruelle, 1969; Martin-Löf, 1979; Georgii, 1995; Touchette, 2011,2015 ). The part of that work relevant to us is the proof ${ }^{52}$ (Touchette, 2015) that if the microcanonical entropy density $s_{\omega}(E, X ; \eta):=S_{\omega}(E, X ; \eta) / \aleph$, using the surface formula $(11.1)$, is a concave function of $E$, then in the thermodynamic limit $s_{\omega}(E, X ; \eta)$ is equal to the entropy density derived from the canonical distribution. ${ }^{53}$ It follows from this that the other thermodynamic quantities are equal. In particular that $T_{\omega}(E, X)$, the temperature derived from $s_{\omega}(E, X ; \eta)$ and given by (11.2), is equal to the canonical temperature $T$. The concavity of $s_{\omega}(E, X ; \eta)$ is just, from (11.4), the positivity of the heat capacity, which has been assumed, and the equivalence of distributions applies both to positive and negative temperatures $T$. Similar results apply to the micromechanical and Gibbs distribution pair.

Of course, experiments are performed on finite not infinite systems and a distinction should be made between small systems and large systems. Obviously these concepts are imprecise and relative to the experimental situation or model calculation under consideration. However, if for the sake of simplicity we take $N:=N$, then for experimental situations one expects $N$ to be of the order of Avogadro's number, that is to say of the order of $10^{23}$. Such systems will in statistical mechanics be regarded as 'large'. Then relative fluctuations are small, meaning that the differences in the results derived using different pairs of distributions (TI-MI/TC-MI and TI-MC/TC-MC) will also be small and the ratios $U / N, S / N, X / N$ etc. will have a negligible dependence on $N$, which disappears in the thermodynamic limit $N \rightarrow \infty$. In particular the limit of $E_{\max } / N$ is finite, which is a stronger condition than that proposed in Sect. 1 . In the light of these results, both at and 'near to' the thermodynamic limit, we argue that statistical mechanics approximately reduces to thermodynamics for large systems, and that arguments in this context based on small systems (where, for example, the difference between $N$ and $N-1$ is significant) should not be regarded a decisive. This should, of course, not be taken as a blanket dismissal of all uses of small systems in statistical mechanics. Real-space renormalization group, numerical simulation and transfer matrix methods all give quite good results for small systems. However, in general, quality increases with size and we are arguing that relative differences in results which decrease with size should not be regarded as significant.

\section{Continuous and Discrete Energy Spectra}

For simplicity we suppose that the lower bound for energy is zero and consider the two cases where the Hamiltonian takes a continuum of values, $E \in\left[0, E_{\max }\right]$ and where it takes a set of discrete values $E_{0}:=0, E_{1}, E_{2}, \ldots, E_{\max }$.

\subsection{A Continuum of Values}

The states of this system are represented by points $x \in \Gamma$, the phase space of the system, and the dynamics is given by a flow which is a semigroup $\left\{\phi_{t} \mid t \geq 0\right\}$ of automorphisms on $\Gamma$, parameterized by time $t \in \mathbb{R}^{+}$. The Hamiltonian of the system $\mathcal{H}(\boldsymbol{x} ; X)$, where $X$ is the one extensive thermodynamic variable, is, for fixed $X$, a constant of the motion, whose value $E$ is the energy of the system. Then all trajectories determined by the flow lie on the energy hypersurface

$$
\Sigma(E, X):=\{\boldsymbol{x} \mid \mathcal{H}(\boldsymbol{x} ; X)=E\} .
$$

Variation of $X$ will alter the Hamiltonian and hence the flow. The density of (energy) states is

$$
\omega(E, X):=\frac{1}{\gamma} \int_{\Gamma} \delta^{\mathrm{Dr}}(E-\mathcal{H}(\boldsymbol{x} ; X)) \mathrm{d} \Gamma
$$

\footnotetext{
${ }^{51}$ This usage of the term 'reduction' related to the thermodynamic limit is that favoured by physicists. Philosophers of science tend to adopt the Nagelian account in which the reverse is the case and thermodynamics reduces to statistical mechanics (Batterman, 2010, p. 160; Sklar, 1993, Chap. 9).

${ }^{52}$ The proof is for a general $N$-particle classical system described by a Hamiltonian which is the energy of the system.

${ }^{53}$ There may of course be a need to adjust the parameter $\eta$.
} 
where $\delta^{\operatorname{Dr}}(\cdot)$ is the Dirac delta function, $\gamma$ is a constant of the dimensions of $\mathrm{d} \Gamma^{54}$ and the integrated density of states is

$$
\Omega(E, X):=\int_{0}^{E} \omega\left(E^{\prime}, X\right) \mathrm{d} E^{\prime}=\frac{1}{\gamma} \int_{\Gamma} \Theta(E-\mathcal{H}(x ; X)) \mathrm{d} \Gamma,
$$

where $\Theta(\cdot)$ is the Heaviside function. Assuming reasonably smooth functions of $E,{ }^{55}$

$$
\omega(E, X)=\frac{\partial \Omega(E, X)}{\partial E} \quad \text { with } \quad v(E, X):=\frac{\partial \omega(E, X)}{\partial E}, \quad \chi(E, X):=\frac{\partial v(E, X)}{\partial E} .
$$

\subsection{A Set of Discrete Values: The $\mathfrak{B}_{m}$ Model}

As an exemplar of a discrete-valued energy system we take a simple quantized model, used by Boltzmann (1877, p. 168), where $N$ non-interacting atoms each has $m$ equally spaced energy levels $0, \varepsilon, 2 \varepsilon, \ldots,(m-1) \varepsilon$, for some energy parameter $\varepsilon>0$ and for some positive integer $m$, with $m=\infty$ a possibility. This is a particular case of the example used in the discussion in Sect. 1.1. The state of the system is given by a point $\boldsymbol{n}:=\left(n_{0}, n_{1}, n_{2}, \ldots, n_{m-1}\right)$ on the hyperplane

$$
\Pi_{N}:=\{\boldsymbol{n} \mid \boldsymbol{n} \cdot \boldsymbol{i}=N\}, \quad \text { where } \boldsymbol{i}:=(1,1, \ldots, 1),
$$

in the space $\Lambda_{m}$ of $m$-dimensional non-negative integer-valued vectors, where $n_{i}$ atoms are in energy level $i \varepsilon$. The Hamiltonian of the assembly is

$$
\mathcal{H}(\boldsymbol{n} ; \boldsymbol{\varepsilon}):=\boldsymbol{\varepsilon} \boldsymbol{n} \cdot \boldsymbol{j}, \quad \text { where } \quad \boldsymbol{j}:=(0,1, \ldots, m-1)
$$

and the energy levels of the assembly are given by

$$
E=\varepsilon \widehat{X}, \quad \text { where } \quad \widehat{X}:=\boldsymbol{n} \cdot \boldsymbol{j}=0,1, \ldots, N(m-1),
$$

specifically between the state $\widehat{X}=0$, where all the atoms are in their ground state and $E=E_{\min }=0$, and $\widehat{X}=N(m-1)$, where all the atoms are in their most excited state and $E=E_{\max }=N(m-1) \varepsilon$. The degeneracy of level $\widehat{X}$ is

$$
\mathscr{W}_{m}(\widehat{X}):=\sum_{\left\{n_{i}\right\}} \frac{\Gamma(N+1)}{\prod_{i=0}^{m-1} \Gamma\left(n_{i}+1\right)},
$$

with the sum over distributions lying on $\Pi_{N}$ and satisfying $\widehat{X}=\boldsymbol{n} \cdot \boldsymbol{j} . \mathscr{W}_{m}(\widehat{X})$, which is defined only for integer values of $\widehat{X}$ in the domain $[0, N(m-1)]$, satisfies $\mathscr{W}_{m}(\widehat{X})=\mathscr{W}_{m}(N(m-1)-\widehat{X})$ with $\mathscr{W}_{m}(0)=\mathscr{W}_{m}(N(m-1))=1$ and has a single maximum when $m$ is odd at $\widehat{X}=\frac{1}{2} N(m-1)$ and two equal maxima when $m$ is even at $\widehat{X}=\frac{1}{2} N(m-1 \pm 1) .{ }^{56}$ The density of energy states is the number of energy states per unit of $\varepsilon$, that is

$$
\omega_{m}(E ; \varepsilon):=\frac{1}{\varepsilon} \mathscr{W}_{m}(E / \varepsilon)
$$

with the summed density of states

$$
\Omega_{m}(E ; \varepsilon):=\varepsilon \sum_{k=0}^{E / \varepsilon} \omega_{m}(k \varepsilon ; \varepsilon)=\sum_{k=0}^{E / \varepsilon} \mathscr{W}_{m}(k)=: \overline{\mathscr{W}}_{m}(E / \varepsilon)
$$

\footnotetext{
${ }^{54}$ In the case of a fluid of $N$ particles in a three-dimensional box the usual choice is $\gamma:=\Gamma(N+1) h^{3 N}$, where $h$ is Planck's constant.

${ }^{55}$ We note that $\Omega(E, X), \omega(E, X)$ and $v(E, X)$ are respectively dimensionless and of dimensions $\mathrm{J}^{-1}$ and $\mathrm{J}^{-2}$.

${ }^{56}$ We pause to note that the cases $m=2,3$ are mathematically equivalent to assemblies of $N$ non-interacting spin- $\frac{1}{2}$ and spin- 1 particles in a magnetic field. (If the ordering of the spin states $\{-1,+1\}$ and $\{-1,0,+1\}$, respectively, is the same as the ordering of states $\{0,1, \ldots, m-1\}$ in this model then the magnetic field is negative.) The former is the example considered by Vilar and Rubi (2014), Campisi (2015, Sect. D) and Abrahm and Penrose (2017), and we shall present results for the latter.
} 
For this discrete-energy system differentiation must be replaced by a finite-difference, and since the smallest incremental difference is $\varepsilon$, for any $F(E ; \varepsilon)$,

$$
\frac{\partial F(E ; \varepsilon)}{\partial E} \equiv \frac{F(E ; \varepsilon)-F(E-\varepsilon ; \varepsilon)}{\varepsilon}
$$

In particular, from (10.10),

$$
\frac{\partial \Omega_{m}(E ; \varepsilon)}{\partial E}=\sum_{k=0}^{E / \varepsilon} \omega_{m}(k \varepsilon ; \varepsilon)-\sum_{k=0}^{E / \varepsilon-1} \omega_{m}(k \varepsilon ; \varepsilon)=\omega_{m}(E ; \varepsilon)
$$

\section{A Thermally Isolated (TI) System: The Choice of Entropy Function}

\subsection{The Continuous-Energy TI-MI System: The Microcanonical Distribution}

Hilbert et al. (2014) have surveyed a range of possibilities; we shall concentrate on the two main contenders.

(i) The surface entropy

$$
S_{\omega}(E, X ; \eta):=\ln [\eta \omega(E, X)]
$$

where $\eta$ is a constant which has the dimensions of energy; thus rendering the argument of the logarithm dimensionless. $S_{\omega}(E, X ; \eta)$ is the most commonly used form for the microcanonical entropy (Huang, 1963, Sect. $6.2),{ }^{57}$ giving, from Table 3 , for the surface temperature and surface expression for the other intensive variable,

$$
\begin{aligned}
& T_{\omega}(E, X)=\left(\frac{\partial S_{\omega}(E, X ; \eta)}{\partial E}\right)^{-1}=\frac{\omega(E, X)}{v(E, X)} \\
& \xi_{\omega}(E, X)=T_{\omega}(E, X) \frac{\partial S_{\omega}(E, X ; \eta)}{\partial X}=\frac{1}{v(E, X)} \frac{\partial \omega(E, X)}{\partial X}
\end{aligned}
$$

and

$$
\begin{aligned}
C_{\omega}(E, X) & =-\left[T_{\omega}(E, X)\right]^{-2}\left(\frac{\partial^{2} S_{\omega}(E, X ; \eta)}{\partial E^{2}}\right)^{-1}=\left(\frac{\partial T_{\omega}(E, X)}{\partial E}\right)^{-1} \\
& =\left(1-\frac{\omega(E, X) \chi(E, X)}{[v(E, X)]^{2}}\right)^{-1}
\end{aligned}
$$

for the surface heat capacity. Khinchin $(1949$, p. 37$)$ refers to $\omega(E, X)$ as the structure function and asserts that it is monotonically increasing in the domain $[0, \infty)$, for $E \in[0, \infty)$. If this were the case the surface temperature would always be positive. Khinchin's English translator comments that "this appears as an additional assumption", and indeed, as we see in the case of the discrete-energy system with a finite number of energy levels, it is not necessarily true.

(ii) The integrated density of states $\Omega(E, X)$ is, of course, a monotonically increasing functions of $E$ and Gibbs (1902, Chap. XIV) proposed the use of the bulk entropy

$$
S_{\Omega}(E, X):=\ln [\Omega(E, X)],
$$

\footnotetext{
${ }^{57}$ Although it should be noted that in the derivation of the equipartition function in his Sect. 6.4 he passes over the problem described in Sect. 14 , below, by using the bulk entropy (11.5).
} 
for a microcanonically distributed system. The bulk equivalents of (11.2)-(11.4) are

$$
\begin{aligned}
T_{\Omega}(E, X) & =\left(\frac{\partial S_{\Omega}(E, X)}{\partial E}\right)^{-1}=\frac{\Omega(E, X)}{\omega(E, X)} \\
\xi_{\Omega}(E, X) & =T_{\Omega}(E, X) \frac{\partial S_{\Omega}(E, X)}{\partial X}=\frac{1}{\omega(E, X)} \frac{\partial \Omega(E, X)}{\partial X} \\
C_{\Omega}(E, X) & =-\left[T_{\Omega}(E, X)\right]^{-2}\left(\frac{\partial^{2} S_{\Omega}(E, X)}{\partial E^{2}}\right)^{-1}=\left(\frac{\partial T_{\Omega}(E, X)}{\partial E}\right)^{-1} \\
& =\left(1-\frac{\Omega(E, X) v(E, X)}{[\omega(E, X)]^{2}}\right)^{-1} .
\end{aligned}
$$

The bulk temperature is always positive with

$$
T_{\omega}(E, X)=\frac{T_{\Omega}(E, X)}{1-\left[C_{\Omega}(E, X)\right]^{-1}},
$$

showing that the surface temperature is negative whenever $C_{\Omega}(E, X) \in[0,1)$.

We also note in passing an intermediate case between the surface and bulk entropies, chosen by some authors (Tolman 1938, Sect. 22; ter Haar 1961, p. 106) as a definition of the microcanonical distribution. This is the shell entropy ${ }^{58}$ where

$$
S_{\mathrm{SH}}(E, X ; \triangle E):=\ln [\Omega(E, X)-\Omega(E-\triangle E, X)], \quad 0<\triangle E .
$$

It is not difficult to show that this, in the limit $\triangle E \rightarrow E$, yields the bulk quantities and, for $\triangle E \ll E$ gives

$$
\begin{aligned}
& S_{\mathrm{SH}}(E, X ; \triangle E) \simeq S_{\omega}(E, X ; \triangle E) \\
& T_{\mathrm{SH}}(E, X ; \triangle E) \simeq T_{\omega}(E, X), \quad \xi_{\mathrm{SH}}(E, X ; \triangle E) \simeq \xi_{\omega}(E, X), \quad C_{\mathrm{SH}}(E, X ; \triangle E) \simeq C_{\omega}(E, X) .
\end{aligned}
$$

To first order in $\triangle E$ the shell entropy coincides with the surface entropy when $\eta:=\triangle E$, which is the value chosen by some authors (Huang 1963, Sect. 7.2; Pathria 1972, Sect. 2.3).

If the Hamiltonian is the only non-trivial ${ }^{59}$ global constant of motion, the energy hypersurface $\Sigma(E, X)$ is an ergodic subspace of the system, meaning that it is indecomposable with respect to the flow. Then the appropriate statistical mechanical distribution for independent variables $E$ and $X$ is given by the microcanonical probability density function $^{60}$

$$
\rho(\boldsymbol{x} \mid E, X):=\frac{\delta^{\mathrm{Dr}}(E-\mathcal{H}(\boldsymbol{x} ; X))}{\gamma \omega(E, X)} .
$$

In a trivial sense

$$
U=E=\langle E \mid E, X\rangle=\langle\mathcal{H}(\boldsymbol{x} ; X) \mid E, X\rangle
$$

\footnotetext{
${ }^{58}$ Hilbert et al. (2014) calls this the 'modified Boltzmann entropy'.

${ }^{59}$ Of course, any constant, or indeed any function of the independent variables, which is not a function of the microscopic phase state is a 'trivial' global constant of motion.

${ }^{60}$ It can also be argued on the basis of the principle of insufficient reason that this is the appropriate distribution when the Hamiltonian is the only known non-trivial global constant of motion.
} 
where $\langle\cdots \mid E, X\rangle$ is calculated using the probability density function (11.13). Less trivially, from (C.2), (C.3), (10.3) and (11.2)-(11.9),

$$
\begin{aligned}
\left\langle\frac{\partial \mathcal{H}(\boldsymbol{x} ; X)}{\partial X} \mid E, X\right\rangle & =-\frac{1}{\omega(E, X)} \frac{\partial \Omega(E, X)}{\partial X}=-T_{\Omega}(E, X) \frac{\partial S_{\Omega}(E, X)}{\partial X}=\xi_{\Omega}(E, X), \\
\left\langle x_{j} \frac{\partial \mathcal{H}(\boldsymbol{x} ; X)}{\partial x_{i}} \mid E, X\right\rangle & =\delta^{\mathrm{Kr}}(i-j) \frac{\Omega(E, X)}{\omega(E, X)}-\mathscr{B}_{i j}(E, X)=\delta^{\mathrm{Kr}}(i-j) T_{\Omega}(E, X)-\mathscr{B}_{i j}(E, X),
\end{aligned}
$$

where $x_{i}$ and $x_{j}$ are any components of $\boldsymbol{x}$, and

$$
\mathscr{B}_{i j}(E, X):=\frac{1}{\gamma \omega(E, X)} \int_{\Gamma} \frac{\partial\left[x_{j} \Theta(E-\mathcal{H}(\boldsymbol{x} ; X))\right]}{\partial x_{i}} \mathrm{~d} \Gamma .
$$

\subsection{The Discrete-Energy TI-MC System: The Micromechanical Distribution}

As we have noted in the footnote on page 31 , the $\mathfrak{B}_{m}$ model is mathematically equivalent to an array of independent spins, where the appropriate $\mathrm{TI}$ distribution is micromechanical with controllable variables $E$, the energy, and a magnetic field. In the $\mathfrak{B}_{m}$ model $\varepsilon$ is an internal energy parameter not a variable, so $E$ is the only controllable variable $^{61}$ with, from (8.2), the enthalpy $H=U=E$ and

$$
X=\widehat{X}=H / \varepsilon \text {. }
$$

\section{The micromechanical probability function is}

$$
\mathrm{P}(\boldsymbol{n} \mid H ; \varepsilon):=\frac{1}{\mathscr{W}_{m}(H / \varepsilon)}
$$

where the degeneracy $\mathscr{W}_{m}(H / \varepsilon)$ is given by (10.8) and the micromechanical surface, bulk and shell entropies are

$$
\begin{array}{rlll}
S_{\omega}(H ; \varepsilon, \eta) & := & \ln \left[\eta \omega_{m}(H ; \varepsilon)\right], \\
S_{\Omega}(H ; \varepsilon) & := & \ln \left[\Omega_{m}(H ; \varepsilon)\right], \\
S_{\mathrm{SH}}(H ; \varepsilon, \triangle H) & := & \ln \left[\Omega_{m}(H ; \varepsilon)-\Omega_{m}(H-\triangle H ; \varepsilon)\right] .
\end{array}
$$

Here the energy states have an incremental spacing $\varepsilon$, making this analogous to $\triangle E$ in the continuous-energy case. So the natural choice is to set $\eta:=\varepsilon=: \triangle H$. Another way to look at the question is to refer back to Boltzmann's intention with respect to the argument of the logarithm. It is supposed (Boltzmann, 1877, p. 169) to be the number of microscopic configurations compatible with a particular macrostate, which in this case is $\mathscr{W}_{m}(H / \varepsilon){ }^{62}$ leading again to $\eta:=\varepsilon$. Substituting from (10.12) into (11.22),

$$
S_{\mathrm{SH}}(H ; \varepsilon, \varepsilon)=S_{\omega}(H ; \varepsilon, \varepsilon)=\ln \left[\mathscr{W}_{m}(H / \varepsilon)\right],
$$

exactly, rather than, as in (11.11), simply for small $\triangle E$. It follows from (10.9) and (10.10) that each of the entropies (11.20)-(11.22) is a function of $H$ through $X=H / \varepsilon$ with

$$
\frac{1}{T}=\frac{\partial S}{\partial H}=\frac{1}{\varepsilon} \frac{\partial S(X)}{\partial X}
$$

For the case of three energy levels (the $\mathfrak{B}_{3}$ model) the surface and bulk entropies are shown in Fig. 10 and the surface and bulk temperatures in Fig. 11. The significance of these results is discussed in Sect. 13.1.

\footnotetext{
${ }^{61}$ The motivation for choosing the micromechanical and Gibbs distributions in this case, rather than the microcanonical and canonical distributions is the need to relate $\mathfrak{B}_{2}$ to the corresponding spin model in Sect. 17.

${ }^{62}$ The objections advanced by Brush (1976, p. 608) to this combinatorial definition: (i) that the states of the microsystems form a continuum, (ii) that the temperature dependence of the model cannot be introduced in this way, are both invalid in the present case since: (i) the microstates are discrete, (ii) the dependence in this $\mathrm{TI}$ system is on $H=E \operatorname{not} T$, and that enters through $\widehat{X}=H / \varepsilon$.
} 


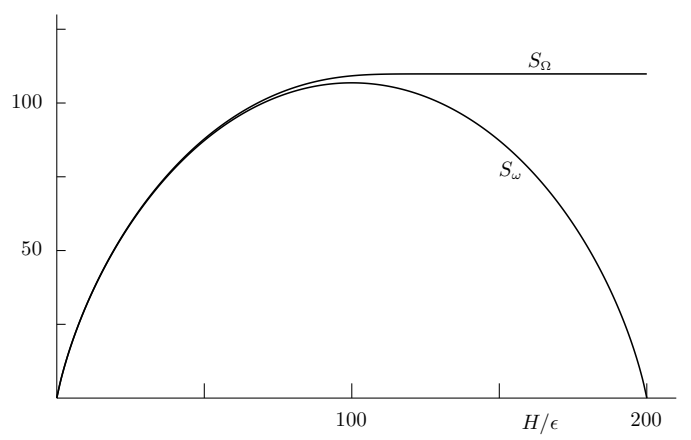

Figure 10: The surface and bulk entropies for the $\mathfrak{B}_{3}$ model with $N=100$.

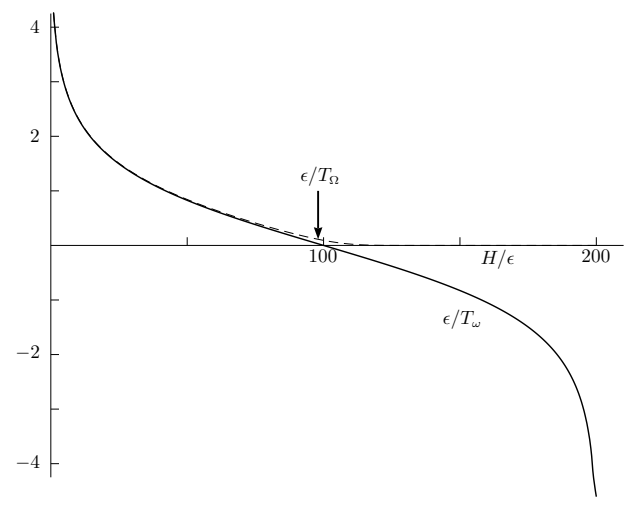

Figure 11: The surface and bulk inverse temperatures for the $\mathfrak{B}_{3}$ model with $N=100$.

For any $N>1$ there is a qualitative change in behaviour from systems with large $m$ to ones with $m \rightarrow \infty$, so that $E_{\max } \rightarrow \infty$. It is not difficult to show that, in this limit,

$$
\mathscr{W}_{\infty}(H / \varepsilon)=\mathscr{W}_{H / \varepsilon}(H / \varepsilon)=\frac{\Gamma(H / \varepsilon+N)}{\Gamma(N) \Gamma(H / \varepsilon+1)} .
$$

Of course, as for the finite $m$ case, $X=H / \varepsilon$ is an integer variable. However, use of gamma functions, avoids, by analytical continuation into real values, the need for numerical differentiation with, from (11.23) and (11.24),

$$
\frac{\varepsilon}{T_{\omega}(H ; \varepsilon)}=\frac{\partial S_{\omega}(X)}{\partial X}=\frac{1}{\mathscr{W}_{\infty}(X)} \frac{\partial \mathscr{W}_{\infty}(X)}{\partial X}=\Psi(X+N)-\Psi(X+1)
$$

where $\Psi(\cdot)$ is the digamma function. The surface and bulk entropies for the infinite case (the $\mathfrak{B}_{\infty}$ model) are shown in Fig. 12 and the surface and bulk temperatures in Fig. 13 .

As we saw in Sect. 1.1, models with no energy upper bound are still capable of yielding negative surface temperatures. A simple example of this was provided by Matty et al. (2017, Sect. V.5), where $\omega(E):=\exp (-v E)$, with $v>0$. In similar way $\mathfrak{B}_{m}$ can be modified by assigning a degeneracy $\exp (-i v)$ to the energy levels $i \varepsilon$, for $i=0,1,2, \ldots$ Results for this model are shown in Figs. 14 and 15. The value of $v$ was chosen to ensure that $S_{\omega}(H / \varepsilon)=0$, when $H=200 \varepsilon$, affording comparison with Figs. 10 and 11 .

\subsection{An Isothermal Reservoir}

This is defined in Sect. 6 as a simple system for which: 


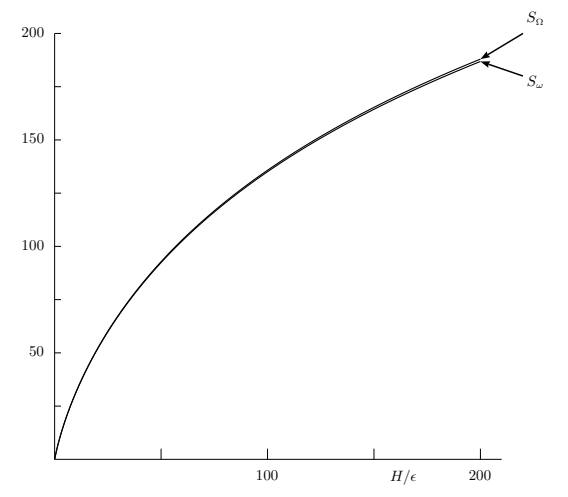

Figure 12: The surface and bulk entropies for the $\mathfrak{B}_{\infty}$ model with $N=100$.

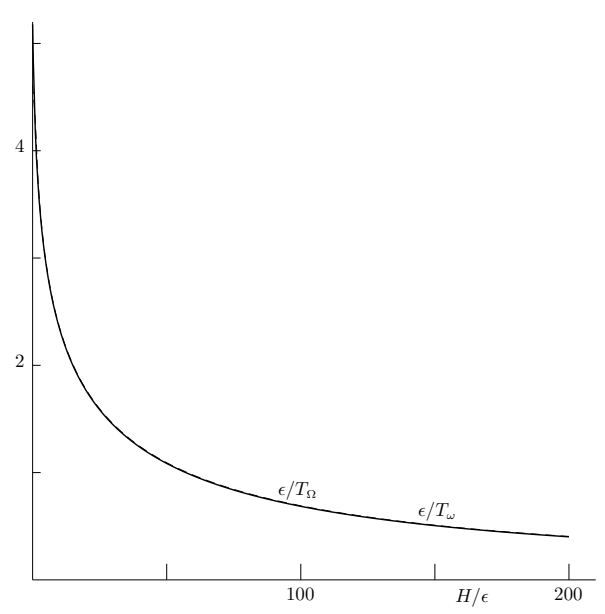

Figure 13: The surface and bulk inverse temperatures for the $\mathfrak{B}_{\infty}$ model with $N=100$.

(a) The deformation variables $X$ and $\xi$ are either absent or kept constant, thus removing the distinction between the microcanonical and micromechanical distributions.

(b) The entropy is a linear function of $E$, meaning that the thermodynamic temperature $T$ has a designated constant value.

Here both $E$ and $T$ can be regarded as independent variables and we use the notation of Sect. 6 to specify the state of the reservoir by $((E ; T))$. For general systems we are interested in the choice between surface and bulk entropies and temperatures. We now investigate how this plays out in the case of an isothermal reservoir. More precisely, whether $T$ is equal to the microcanonical surface temperature or bulk temperature when the reservoir is thermally isolated. For the sake of this discussion, let $E \in\left[E_{\min }, E_{\max }\right]$, so that the zero lower bound in the integral in (10.3) is replaced by $E_{\min }$ with $\Omega\left(E_{\min } ; T\right)=0$.

First suppose that $T_{\omega}(E ; T)=T$. Then $\omega^{\prime}(E ; T)=\omega(E ; T) / T$, giving, from (10.3), (10.4), (11.1), (11.2), (11.5) and 


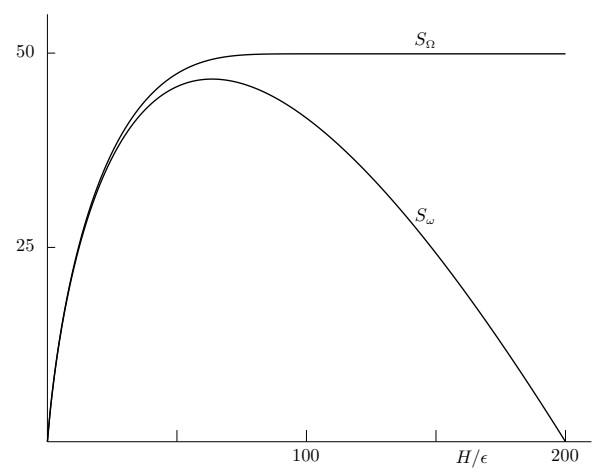

Figure 14: The surface and bulk entropies for the modified $\mathfrak{B}_{\infty}$ model with $v=0.9341794$ and $N=100$.

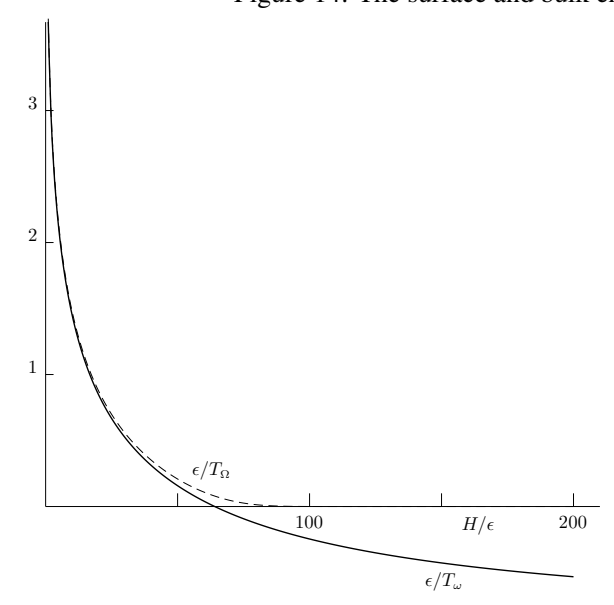

Figure 15: The surface and bulk inverse temperatures for the modified $\mathfrak{B}_{\infty}$ model with $v=0.9341794$ and $N=100$.

(11.6) (and using suitable subscripts).

$$
\begin{array}{ll}
\Omega_{\omega}(E ; T)=\omega_{0} T\left\{\exp (E / T)-\exp \left(E_{\min } / T\right)\right\}, & \omega_{\omega}(E ; T)=\omega_{0} \exp (E / T), \\
v_{\omega}(E ; T)=\omega_{0} \exp (E / T) / T, & \chi_{\omega}(E ; T)=\omega_{0} \exp (E / T) / T^{2}, \\
S_{\omega}(E ; T, \eta)=\ln \left(\eta \omega_{0}\right)+E / T, & S_{\Omega}(E ; T)=\ln \left(\omega_{0}\right)+\ln \left[T\left\{\exp (E / T)-\exp \left(E_{\min } / T\right)\right\}\right], \\
T_{\Omega}(E ; T)=T\left\{1-\exp \left(\left[E_{\min }-E\right] / T\right)\right\}, &
\end{array}
$$

for some constant $\omega_{0}$.

On the other hand, if $T_{\Omega}(E ; T)=T$, then $\Omega^{\prime}(E ; T)=\Omega(E ; T) / T$, giving

$$
\begin{array}{ll}
\Omega_{\Omega}(E ; T)=\Omega_{0} \exp (E / T), & \omega_{\Omega}(E ; T)=\Omega_{0} \exp (E / T) / T, \\
v_{\Omega}(E ; T)=\Omega_{0} \exp (E / T) / T^{2}, & \chi_{\Omega}(E ; T)=\Omega_{0} \exp (E / T) / T^{3}, \\
S_{\Omega}(E ; T)=\ln \left(\Omega_{0}\right)+E / T, & S_{\omega}(E ; T, \eta)=\ln \left(\eta \Omega_{0} / T\right)+E / T, \\
T_{\omega}(E ; T)=T, &
\end{array}
$$

for some constant $\Omega_{0}$. 
$\Omega_{\Omega}(E ; T)$ satisfies the boundary condition $\Omega_{\Omega}\left(E_{\min } ; T\right)=0$ only when $E_{\min }=-\infty$ and $T>0$; then with $\Omega_{0}:=\omega_{0} T$, the sets of equations (11.27) and (11.28) become identical. They are also in asymptotic agreement in the limit $E \rightarrow \infty$, when $T>0, E_{\max }=\infty$. It can reasonably be argued that a reservoir which is truly large with respect to any finite system requires that $E \in[-\infty, \infty]$. However, the point of our definition of an isothermal reservoir is to avoid the need for largeness and in any event the restriction to positive temperatures is more serious in the light of our use of a reservoir at a negative temperature in Sect. 6.

We conclude that the thermodynamic temperature $T$ of the reservoir can be identified with the microcanonical temperature for the surface entropy, but for the bulk entropy, only for $E_{\min }=-\infty$ and positive temperatures, or asymptotically for large energies and positive temperatures. Which returns us to the traditional idea of a heat reservoir, which, as described in Sect. 6, is taken to be so large that its state is unaffected by flows of energy into and out of a system with which it is in thermal contact. In all the following discussion, since we are concerned to accommodate negative temperatures, we chose $E_{\min }$ to be finite, in fact, without loss of generality $E_{\min }:=0$, and implicitly assume that $E_{\max }:=\infty$ when an isothermal reservoir is treated from the perspective of the bulk entropy.

\section{Systems in Thermal Contact (TC) with their Environment}

\subsection{The Continuous-Energy TC-MI Case: The Canonical Distribution}

As indicated at the beginning of Part I, classical thermodynamics is concerned with systems in equilibrium, both a single system $\Xi$, scaled versions $\Xi(\lambda)$ of $\Xi$ and Cartesian products $\Xi_{\mathrm{A}} \times \Xi_{\mathrm{B}}$ of such systems, which can simply be pictured as the two systems $\Xi_{\mathrm{A}}$ and $\Xi_{\mathrm{B}}$ taken together, but with no interaction between them, so that, if $X_{\mathrm{A}} \in \Xi_{\mathrm{A}}$ and $X_{\mathrm{B}} \in \Xi_{\mathrm{B}},\left(X_{\mathrm{A}}, X_{\mathrm{B}}\right) \in \Xi_{\mathrm{A}} \times \Xi_{\mathrm{B}}$. There is also the need to consider the situation when two systems $\Xi_{\mathrm{A}}$ and $\Xi_{\mathrm{B}}$ are brought into thermal contact. ${ }^{63}$ Given that they are otherwise isolated, an adiabatic process of thermal equilibration will, as described in Sect. 5, occur (unless the systems are already at equilibrium) and the extremum condition imposed on (5.3) leads to the equal temperatures condition (5.4) and the entropy conditions (5.5), for the cases where $S(+)$ and $\mathrm{S}(-)$ respectively apply. It should be emphasised that thermal equilibrium in this context is for the individual systems, that is to say for $\Xi_{\mathrm{A}}$ and $\Xi_{\mathrm{B}}$ when they are individually isolated. Thus it applies after splitting following a joining, when equilibration may or may not have been necessary.

Approaching the problem from a statistical mechanical standpoint, suppose that $\Xi_{\mathrm{A}}$ and $\Xi_{\mathrm{B}}$ are thermally joined so that the joined system $\Xi_{\mathrm{AB}}$ has total energy $E_{\mathrm{T}}$. The energy $E_{\mathrm{A}}$ of $\Xi_{\mathrm{A}}$ will fluctuate in the range $\left[0, E_{\mathrm{T}}\right]$, with conditional probability density function given from (C.6) by

$$
\rho_{\mathrm{A}}\left(E_{\mathrm{A}} \mid X_{\mathrm{A}}, X_{\mathrm{B}} ; E_{\mathrm{T}}\right):=\frac{\omega_{\mathrm{A}}\left(E_{\mathrm{A}}, X_{\mathrm{A}}\right) \omega_{\mathrm{B}}\left(E_{\mathrm{T}}-E_{\mathrm{A}}, X_{\mathrm{B}}\right)}{\omega_{\mathrm{AB}}\left(E_{\mathrm{T}}, X_{\mathrm{A}}, X_{\mathrm{B}}\right)}=\rho_{\mathrm{B}}\left(E_{\mathrm{T}}-E_{\mathrm{A}} \mid X_{\mathrm{B}}, X_{\mathrm{A}} ; E_{\mathrm{T}}\right) .
$$

The expected value of the bulk temperature of $\Xi_{\mathrm{A}}$ is, from (11.7) and (C.8), given by

$$
\left\langle T_{\Omega \mathrm{A}}\left(E_{\mathrm{A}}, X_{\mathrm{A}}\right) \mid X_{\mathrm{A}}, X_{\mathrm{B}} ; E_{\mathrm{T}}\right\rangle=\int_{0}^{E_{\mathrm{T}}} \mathrm{d} E_{\mathrm{A}} \frac{\Omega_{\mathrm{A}}\left(E_{\mathrm{A}}, X_{\mathrm{A}}\right) \omega_{\mathrm{B}}\left(E_{\mathrm{T}}-E_{\mathrm{A}}, X_{\mathrm{B}}\right)}{\omega_{\mathrm{AB}}\left(E_{\mathrm{T}}, X_{\mathrm{A}}, X_{\mathrm{B}}\right)}=T_{\Omega \mathrm{AB}}\left(E_{\mathrm{T}}, X_{\mathrm{A}}, X_{\mathrm{B}}\right) .
$$

and, of course, by the same argument,

$$
\left\langle T_{\Omega \mathrm{A}}\left(E_{\mathrm{A}}, X_{\mathrm{A}}\right) \mid X_{\mathrm{A}}, X_{\mathrm{B}} ; E_{\mathrm{T}}\right\rangle=\left\langle T_{\Omega \mathrm{B}}\left(E_{\mathrm{B}}, X_{\mathrm{B}}\right) \mid X_{\mathrm{B}}, X_{\mathrm{A}} ; E_{\mathrm{T}}\right\rangle=T_{\Omega \mathrm{AB}}\left(E_{\mathrm{T}}, X_{\mathrm{A}}, X_{\mathrm{B}}\right) .
$$

From (11.3) and (C.7) ${ }^{64}$ the corresponding expressions for the surface quantities are

$$
\begin{aligned}
& \left\langle\frac{1}{T_{\omega \mathrm{A}}\left(E_{\mathrm{A}}, X_{\mathrm{A}}\right)} \mid X_{\mathrm{A}}, X_{\mathrm{B}} ; E_{\mathrm{T}}\right\rangle=\int_{0}^{E_{\mathrm{T}}} \mathrm{d} E_{\mathrm{A}} \frac{v_{\mathrm{A}}\left(E_{\mathrm{A}}, X_{\mathrm{A}}\right) \omega_{\mathrm{B}}\left(E_{\mathrm{T}}-E_{\mathrm{A}}, X_{\mathrm{B}}\right)}{\omega_{\mathrm{AB}}\left(E_{\mathrm{T}}, X_{\mathrm{A}}, X_{\mathrm{B}}\right)}=\frac{1}{T_{\omega_{\mathrm{AB}}}\left(E_{\mathrm{T}}, X_{\mathrm{A}}, X_{\mathrm{B}}\right)}, \\
& \left\langle\frac{1}{T_{\omega \mathrm{A}}\left(E_{\mathrm{A}}, X_{\mathrm{A}}\right)} \mid X_{\mathrm{A}}, X_{\mathrm{B}} ; E_{\mathrm{T}}\right\rangle=\left\langle\frac{1}{T_{\omega \mathrm{B}}\left(E_{\mathrm{B}}, X_{\mathrm{B}}\right)} \mid X_{\mathrm{A}}, X_{\mathrm{B}} ; E_{\mathrm{T}}\right\rangle=\frac{1}{T_{\omega \mathrm{AB}}\left(E_{\mathrm{T}}, X_{\mathrm{A}}, X_{\mathrm{B}}\right)} .
\end{aligned}
$$

\footnotetext{
${ }^{63}$ As indicated in the footnote on page 17 , there is a need to consider both actual and potential contact.

${ }^{64}$ Assuming in this case that $\omega_{\mathrm{B}}(0+)=0$.
} 
Now suppose that $\Xi_{\mathrm{B}}$ is an isothermal reservoir at temperature $T$ with its state and character defined, as in Sect. 11.3, by $\left(\left(E_{\mathrm{B}} ; T\right)\right) ; X_{\mathrm{B}}$ is a constant which can be ignored. From (11.27), (11.28), (12.3) and (12.5)

$$
T_{\omega \mathrm{B}}\left(E_{\mathrm{B}}\right)=T_{\omega \mathrm{AB}}\left(E_{\mathrm{T}}, X_{\mathrm{A}}\right)=T
$$

for positive or negative $T$, and, asymptotically for large $E$, and

$$
T_{\Omega \mathrm{B}}\left(E_{\mathrm{B}}\right)=T_{\Omega \mathrm{AB}}\left(E_{\mathrm{T}}, X_{\mathrm{A}}\right)=T
$$

for positive $T$, with

$$
\rho_{\mathrm{A}}\left(E_{\mathrm{A}} \mid T, X_{\mathrm{A}} ; E_{\mathrm{T}}\right)=\frac{\eta \omega_{0} \omega_{\mathrm{A}}\left(E_{\mathrm{A}}, X_{\mathrm{A}}\right) \exp \left[\left(E_{\mathrm{T}}-E_{\mathrm{A}}\right) / T\right]}{\omega_{\mathrm{AB}}\left(E_{\mathrm{T}}, X_{\mathrm{A}}\right)}=\frac{\omega_{\mathrm{A}}\left(E_{\mathrm{A}}, X_{\mathrm{A}}\right) \exp \left(-E_{\mathrm{A}} / T\right)}{Z_{\mathrm{C}}^{\prime}\left(T, X_{\mathrm{A}} ; E_{\mathrm{T}}\right)},
$$

where

$$
Z_{\mathrm{C}}^{\prime}\left(T, X_{\mathrm{A}} ; E_{\mathrm{T}}\right):=\left(\eta \omega_{0}\right)^{-1} \omega_{\mathrm{AB}}\left(E_{\mathrm{T}}, X_{\mathrm{A}}\right) \exp \left(-E_{\mathrm{T}} / T\right)=\int_{0}^{E_{\mathrm{T}}} \omega_{\mathrm{A}}\left(E_{\mathrm{A}}, X_{\mathrm{A}}\right) \exp \left(-E_{\mathrm{A}} / T\right) \mathrm{d} E_{\mathrm{A}} .
$$

It has been assumed that the total system $\Xi_{\mathrm{AB}}$ is isolated with fixed total energy $E_{\mathrm{T}}$, which is the upper bound $E_{\mathrm{max}}$ on the energy in the form of heat provided by the heat bath/reservoir and available to $\Xi_{\mathrm{A}}$. Dropping the subscript 'A', (12.8) is replaced by

$$
\rho\left(E \mid T, X ; E_{\max }\right)=\frac{\omega(E, X) \exp (-E / T)}{Z_{\mathrm{C}}\left(T, X ; E_{\max }\right)},
$$

where

$$
Z_{\mathrm{C}}\left(T, X ; E_{\max }\right):=\int_{0}^{E_{\max }} \omega(E, X) \exp (-E / T) \mathrm{d} E .
$$

These are respectively the canonical probability density and partition functions with respect to which

$$
\begin{aligned}
U & =\left\langle E \mid T, X ; E_{\max }\right\rangle=\frac{\int_{0}^{E_{\max }} \omega(E, X) E \exp (-E / T) \mathrm{d} E}{Z_{\mathrm{C}}\left(T, X ; E_{\max }\right)} \\
& =T^{2} \frac{\partial \ln \left[Z_{\mathrm{C}}\left(T, X ; E_{\max }\right)\right]}{\partial T}=-T \ln \left[Z_{\mathrm{C}}\left(T, X ; E_{\max }\right)\right]-T \frac{\partial\left\{-T \ln \left[Z_{\mathrm{C}}\left(T, X ; E_{\max }\right)\right]\right\}}{\partial T},
\end{aligned}
$$

establishing, from Table 3, the expression

$$
A\left(T, X ; E_{\max }\right):=-T \ln \left[Z_{\mathrm{C}}\left(T, X ; E_{\max }\right)\right]
$$

for the Helmholtz free energy. It is important to note that the derivation of the canonical probability density function is predicated on the form chosen for the microcanonical entropy. In the case of the choice of the bulk entropy it is valid only for $T>0$ and $E_{\max }=\infty$. In particular, from (12.4) and (12.6),

$$
\frac{1}{T}=\left\langle\frac{1}{T_{\omega}(E, X)} \mid T, X ; E_{\max }\right\rangle=\int_{0}^{E_{\max }} \frac{\rho\left(E \mid T, X ; E_{\max }\right)}{T_{\omega}(E, X)} \mathrm{d} E
$$

and, from (12.2) and (12.7), for $T>0$ and $E_{\max }=\infty$,

$$
T=\left\langle T_{\Omega}(E, X) \mid T, X\right\rangle=\int_{0}^{\infty} T_{\Omega}(E, X) \rho(E \mid T, X) \mathrm{d} E .
$$


For a phase function $F(\boldsymbol{x} ; X)$ the canonical expectation is calculated using

$$
\tilde{\rho}\left(\boldsymbol{x} \mid T, X ; E_{\max }\right):=\frac{\exp (-\mathcal{H}(\boldsymbol{x} ; X) / T)}{\gamma Z_{\mathrm{C}}\left(T, X ; E_{\max }\right)}=\int_{0}^{E_{\max }} \rho(\boldsymbol{x} \mid E, X) \rho\left(E \mid T, X ; E_{\max }\right) \mathrm{d} E .
$$

And thus, from (11.15) (and again only for $T>0$ and $E_{\max }=\infty$ ),

$$
\xi_{\mathrm{c}}(T, X)=\frac{\partial A(T, X)}{\partial X}=\left\langle\frac{\partial \mathcal{H}(\boldsymbol{x} ; X)}{\partial X} \mid T, X\right\rangle=\int_{0}^{\infty} \xi_{\Omega}(E, X) \rho(E \mid T, X) \mathrm{d} E .
$$

In Sect. 18 we show that the relationships (11.15) and (12.17) for the bulk intensive variable $\xi_{\Omega}(E, X)$ hold in the thermodynamic limit for the surface intensive variable $\xi_{\omega}(E, X)$.

\subsection{The Discrete-Energy TC-MC Case: The Gibbs Distribution}

For the $\mathfrak{B}_{m}$ model, the controllable variable is now $T$. The fluctuating quantity is the vector $\boldsymbol{n}$, where since, from (10.7), $\widehat{X}=\boldsymbol{n} . \boldsymbol{j}$ and $E=\varepsilon \widehat{X}$, both $\widehat{X}$ and $E$ fluctuate with the enthalpy given by

$$
H=\langle\mathcal{H}(\boldsymbol{n} ; \varepsilon) \mid T ; \varepsilon\rangle=\langle\varepsilon \widehat{X} \mid T ; \varepsilon\rangle,
$$

where $\langle\cdots \mid T ; \varepsilon\rangle$ is calculated using the Gibbs probability function

$$
\mathrm{P}(\widehat{X} \mid T ; \varepsilon):=\frac{\mathscr{W}_{m}(\widehat{X}) \exp (-\varepsilon \widehat{X} / T)}{Z_{\mathrm{G}}(T ; \varepsilon)}
$$

and

$$
Z_{\mathrm{G}}(T ; \varepsilon):=\sum_{\widehat{X}=0}^{N(m-1)} \mathscr{W}_{m}(\widehat{X}) \exp (-\varepsilon \widehat{X} / T)
$$

is the Gibbs partition function. From (12.18)-(12.19),

$$
H=T^{2} \frac{\partial \ln \left[Z_{\mathrm{G}}(T ; \varepsilon)\right]}{\partial T}=-T \ln \left[Z_{\mathrm{G}}(T ; \varepsilon)\right]-T \frac{\partial\left\{-T \ln \left[Z_{\mathrm{G}}(T ; \varepsilon)\right]\right\}}{\partial T},
$$

thereby establishing, from Table 3, the expression

$$
G(T ; \varepsilon):=-T \ln \left[Z_{\mathrm{G}}(T ; \varepsilon)\right]
$$

for the Gibbs free energy.

\section{Relating Distributions}

Comparisons between distributions provides an important element in the discussion of the rival claims of the surface and bulk entropies (Buonsante et al., 2016; Matty et al., 2017). In Sect. 9 we presented the evidence for the equivalence in the thermodynamic limit of the pairs of distributions microcanonical/canonical and micromechanical/Gibbs away from critical regions and with positive heat capacities. We now examine the situation for large finite systems in more detail for both surface and bulk entropies. 


\subsection{The Microcanonical and Canonical Distributions}

In the microcanonical distribution the controllable variables are the mechanical energy $E$ and the deformation variable $X$ with the internal energy $U:=E$. With given density of states $\omega(E, X)$ the surface and bulk entropies are calculated from (11.1) and (11.5) and the surface and bulk temperatures $T_{\omega}(E, X)$ and $T_{\Omega}(E, X)$ from (11.2) and (11.6). In the canonical distribution the controllable variables are the temperature $T$ and $X$ and, again given the density of states, the Helmholtz free energy can be calculated from (12.11) and (12.13). Given that, for the reasons we present above, we expect the distributions to be approximately equivalent for large systems we have two possible equivalences for temperature

$$
T_{\omega}\left(\left\langle E \mid T, X ; E_{\max }\right\rangle, X\right)=T \quad \text { and } \quad T_{\Omega}\left(\left\langle E \mid T, X ; E_{\max }\right\rangle, X\right)=T,
$$

where, of course, in the latter case $E_{\max }=\infty$ and possible validity is restricted to the range $T>0$. The results for the perfect fluid are given in (D.14), where we see that the condition holds exactly for the bulk temperature and in the thermodynamic limit, where the size parameter $N \rightarrow \infty$, for the surface temperature. From (12.10),

$$
\left\langle E \mid T, X ; E_{\max }\right\rangle=\frac{\int_{0}^{E_{\max }} E \exp \left[\zeta_{\omega}(E ; T, X ; \eta)\right] \mathrm{d} E}{\int_{0}^{E_{\max }} \exp \left[\zeta_{\omega}(E ; T, X ; \eta)\right] \mathrm{d} E},
$$

where

$$
\zeta_{\omega}(E ; T, X ; \eta):=S_{\omega}(E, X ; \eta)-E / T \text {. }
$$

Given that $S_{\omega}(E, X ; \eta)$ is a concave non-negative function of $E$ with $S_{\omega}(0, X ; \eta)=0$, as in Figs. 2(a),(b), $\zeta_{\omega}(E ; T, X ; \eta)$ has a single maximum at $E=E^{\star}(T, X)$ given by

$$
\left(\frac{\partial \zeta_{\omega}(E ; T, X ; \eta)}{\partial E}\right)^{\star}=\left(\frac{\partial S_{\omega}(E, X ; \eta)}{\partial E}\right)^{\star}-\frac{1}{T}=\frac{1}{T_{\omega}\left(E^{\star}, X\right)}-\frac{1}{T}=0
$$

Assuming that $E^{\star} \in\left[0, E_{\max }\right]$ it follows from Laplace's method ${ }^{65}$ that

$$
\left\langle E \mid T, X ; E_{\max }\right\rangle \stackrel{\text { TL }}{\simeq} E^{\star} \quad \Longrightarrow \quad T_{\omega}\left(\left\langle E \mid T, X ; E_{\max }\right\rangle, X\right) \stackrel{\text { IL }}{\simeq} T,
$$

which is not an unexpected result since fluctuations around $\left\langle E \mid T, X ; E_{\max }\right\rangle$ decrease with system size with the exact coincidence between the canonical temperature and the microcanonical surface temperature in the thermodynamic limit. This leaves open the question of the behaviour of the bulk temperature. For this we adopt the procedure of Buonsante et al. (2016). We have already noted the difficulty of developing the canonical distribution using the bulk entropy in the case where the energy domain has a finite upper bound. However, this can be set aside in the present discussion since it is clear that $\omega(E, X)$ and $\Omega(E, X)$ have the same domain of energy $\left[0, E_{\max }\right]$, where $E_{\max }$ may or may not be finite, and we are concerned only with comparing the surface and bulk temperatures in the microcanonical distribution for $E \in\left[0, E_{\max }\right]$. Since, as already indicated, $S_{\omega}(E, X ; \eta)$ is a concave non-negative function of $E$ with $S_{\omega}(0, X ; \eta)=0$, there are two possibilities:

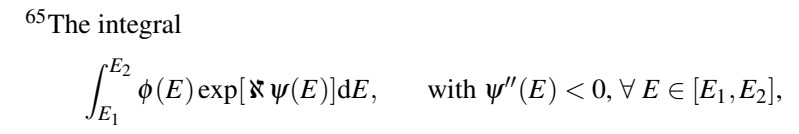

is asymptotically equal to

$$
\begin{aligned}
-\phi\left(E_{1}\right) \exp \left[\aleph \psi\left(E_{1}\right)\right] /\left[\aleph \psi^{\prime}\left(E_{1}\right)\right], & \text { if } \psi^{\prime}(E)<0, \forall E \in\left[E_{1}, E_{2}\right], \\
\phi\left(E_{2}\right) \exp \left[\aleph \psi\left(E_{2}\right)\right] /\left[\aleph \psi^{\prime}\left(E_{2}\right)\right], & \text { if } \psi^{\prime}(E)>0, \forall E \in\left[E_{1}, E_{2}\right], \\
\phi\left(E^{\star}\right) \exp \left[\aleph \psi\left(E^{\star}\right)\right] \sqrt{2 \pi /\left|\aleph \psi^{\prime \prime}\left(E^{\star}\right)\right|} & \text { if } \psi^{\prime}\left(E^{\star}\right)=0, \text { for some } E^{\star} \in\left(E_{1}, E_{2}\right),
\end{aligned}
$$

in the limit $\aleph \rightarrow \infty$ (Copson, 1967, Chap. 5). In the present context, where $\mathfrak{k}$ is the size parameter, this is the thermodynamic limit and asymptotic equality is denoted by $\stackrel{\mathrm{TL}}{\simeq}$. 
(i) $S_{\omega}(E, X ; \eta)$ is an increasing function, and in consequence $T_{\omega}^{-1}(E, X)>0, \forall E \in\left[0, E_{\max }\right]$.

(ii) $S_{\omega}(E, X ; \eta)$ attains a maximum with $T_{\omega}^{-1}(E, X)=0$, for some $\stackrel{\circ}{E} \in\left(0, E_{\max }\right)$.

From (10.3), and again applying Laplace's method, for $E \leq E_{\max }$,

$$
\Omega(E, X)=\frac{1}{\eta} \int_{0}^{E} \exp \left[S_{\omega}\left(E^{\prime}, X ; \eta\right)\right] \mathrm{d} E^{\prime} \stackrel{\mathrm{TL}}{\simeq}\left\{\begin{array}{c}
T_{\omega}(E, X) \exp \left[S_{\omega}(E, X ; \eta)\right] / \eta, \\
\text { if } T_{\omega}^{-1}\left(E^{\prime}, X\right)>0, \forall E^{\prime} \in[0, E], \\
T_{\omega}(\stackrel{\circ}{E}, X) \sqrt{2 \pi C_{X \omega}(\stackrel{\circ}{E}, X)} \exp \left[S_{\omega}(\stackrel{\circ}{E}, X ; \eta)\right] / \eta, \\
\quad \text { if } T_{\omega}^{-1}(\stackrel{\circ}{E}, X)=0, \text { for some } \stackrel{\circ}{E} \in(0, E) .
\end{array}\right.
$$

It follows that

$$
S_{\Omega}(E, X) \stackrel{\text { TL }}{\simeq} \begin{cases}S_{\omega}(E, X ; \eta) & \text { if } T_{\omega}^{-1}(E, X)>0, \\ S_{\omega}(\stackrel{\circ}{E}, X ; \eta) & \text { if } T_{\omega}^{-1}(E, X) \leq 0 .\end{cases}
$$

And to summarize the results of this section

$$
\begin{array}{ll}
T_{\omega}(E, X) \stackrel{\mathrm{TL}}{\simeq} T_{\Omega}(E, X) \stackrel{\mathrm{TL}}{\simeq} T, & \text { if } T>0, \\
T_{\omega}(E, X) \stackrel{\mathrm{TL}}{\simeq} T \quad \text { and } \quad T_{\Omega}(E, X)=\infty, & \text { if } T<0 .
\end{array}
$$

\subsection{The Micromechanical and Gibbs Distributions}

In the micromechanical distribution for the $\mathfrak{B}_{m}$ model the controllable variable is $E$, with the enthalpy $H=E$ and in the Gibbs distribution the controllable variable is $T$. The possibilities (13.1) to test the equivalence of distributions are replaced by

$$
T_{\omega}(\langle E \mid T ; \varepsilon\rangle ; \varepsilon)=T \quad \text { and } \quad T_{\Omega}(\langle E \mid T ; \varepsilon\rangle ; \varepsilon)=T
$$

The expectation value $\langle E \mid T ; \varepsilon\rangle$ can now be calculated from (10.9), (11.20) and (12.19), or, since the total energy is no longer constrained, it follows quite simply that

$$
\langle E \mid T ; \varepsilon\rangle=\frac{N \varepsilon \sum_{i=0}^{m-1} i \exp (-i \varepsilon / T)}{\sum_{i=0}^{m-1} \exp (-i \varepsilon / T)}=\frac{N \varepsilon\{\exp (-\varepsilon / T)-m \exp (-m \varepsilon / T)+(m-1) \exp (-[m+1] \varepsilon / T)\}}{\{1-\exp (-\varepsilon / T)\}\{1-\exp (-m \varepsilon / T)\}} .
$$

In using this result to test (13.9) there is a technical problem to overcome. This is because $T_{\omega}(E ; \varepsilon)$ and $T_{\Omega}(E ; \varepsilon)$ are, in the micromechanical distribution, defined only for values of $E$ in the set $\{0, \varepsilon, 2 \varepsilon, \ldots, N(m-1) \varepsilon\}$ and the computation of $\langle E \mid T ; \varepsilon\rangle$, using (13.10), with an arbitrary choice of $T$, will not necessarily produce a value in this set. However, it is easy to see that the conditions

$$
\left\langle E \mid T_{\omega}(E ; \varepsilon) ; \varepsilon\right\rangle=E \quad \text { and } \quad\left\langle E \mid T_{\Omega}(E ; \varepsilon) ; \varepsilon\right\rangle=E
$$

are equivalent to (13.9) and the test is implemented by plotting the left-hand sides of these formulae as functions of $E$. Or more precisely, since $\left\langle E \mid T_{\omega}(E ; \varepsilon) ; \varepsilon\right\rangle / \varepsilon$ and $\left\langle E \mid T_{\Omega}(E ; \varepsilon) ; \varepsilon\right\rangle / \varepsilon$ are functions of $E$ and $\varepsilon$ only through the ratio $E / \varepsilon$, as functions of this ratio. The results for the case $m=3$ are shown in Fig. 16 and for an infinite number of energy levels in Fig. 17. For large systems verification of (13.11) corresponds to approximate straight line graphs of unit gradient. We observe that this condition is satisfied, for all $E$ and $m=3$, by the surface, but not the bulk, formula. In the latter case $\left\langle E \mid T_{\Omega}(E ; \varepsilon) ; \varepsilon\right\rangle \stackrel{\mathrm{TL}}{\simeq}\left\langle E \mid T_{\Omega}(100 \varepsilon ; \varepsilon) ; \varepsilon\right\rangle \forall E \geq 100 \varepsilon$. This result is in keeping with the conclusions for 


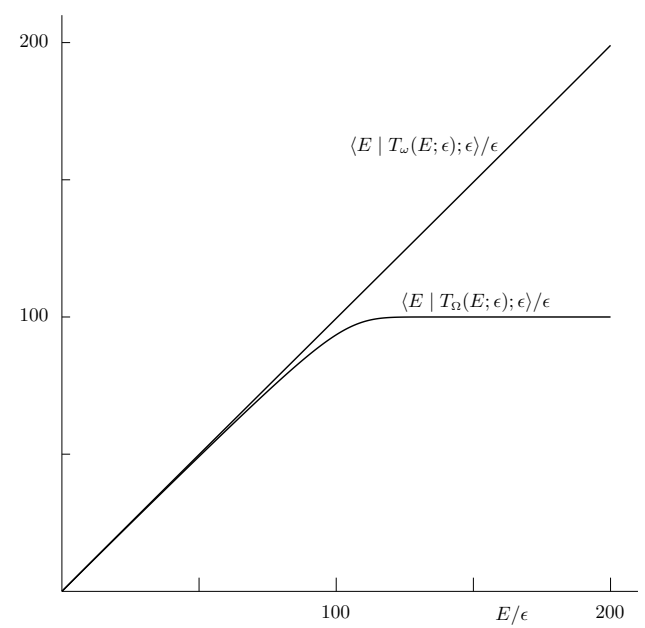

Figure 16: Test of (13.11) for an assembly of $N=100$ atoms with three energy levels.

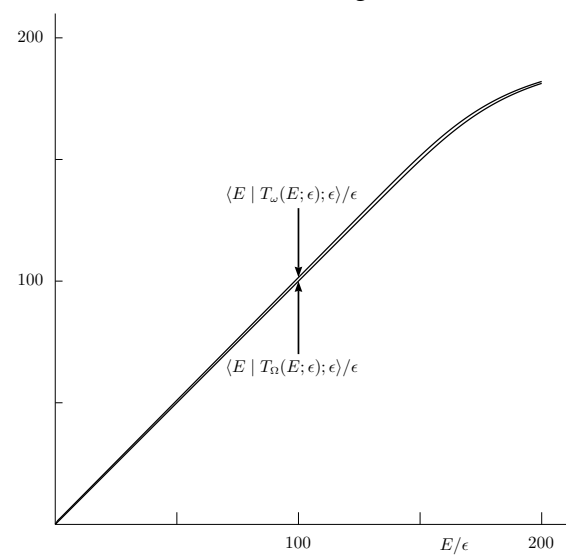

Figure 17: Test of (13.11) for an assembly of $N=100$ atoms with an infinite number of energy levels.

the continuous-energy case in Sect. 13.1, as are also the curves for entropy and inverse temperature displayed in Figs. 10 and 11.

\section{Part III}

\section{Issues of Contention}

\section{The Equipartition Theorem}

From (11.16) and (13.8),

$$
\left\langle x_{j} \frac{\partial \mathcal{H}(\boldsymbol{x} ; X)}{\partial x_{i}} \mid E, X\right\rangle=\mathscr{B}_{i j}(E, X)+ \begin{cases}0, & \text { if } i \neq j \text { and } T>0 \\ T_{\Omega}(E, X), & \text { if } i=j \text { and } T>0 \\ \infty, & \forall i, j \text { and } T<0\end{cases}
$$

where $\mathscr{B}_{i j}(E, X)$ is given by $(11.17)$ and $T_{\Omega}(E, X) \stackrel{\text { th }}{\simeq} T_{\omega}(E, X)$ for $T>0$. The case $\mathscr{B}_{i j}(E, X)=0, \forall i, j$ and $T>0$ is the 'normal' version of the equipartition theorem (Huang, 1963, Sect. 7.4), satisfied exactly by the bulk temperature 
$T_{\Omega}(E, X)$ and asymptotically by the surface temperature $T_{\omega}(E, X)$ in the thermodynamic limit. We have showed in Appen. D that this is satisfied in the perfect fluid which has only positive temperatures. Irrespective of whether $\mathscr{B}_{i j}(E, X)$ is zero or not the equipartition theorem does not hold where the surface and bulk temperatures do not agree in the thermodynamic limit, that is to say when $T<0$. The normal form of the equipartition function is dependent on the model-dependent integral $\mathscr{B}_{i j}(E, X)$ being zero for all $i$ and $j$. Since this term is dependent on the boundary of $\Gamma$ one might suppose (Buonsante et al., 2016) that it vanishes, as in the case of a perfect fluid, for all systems with unbounded energy, yielding the normal equipartition result. Even if this were so, there remains the case where the energy domain has an upper bound. However, as for the $\mathfrak{B}_{m}$ model, such systems are likely to exhibit both negative and positive temperatures. For the normal version of equipartition to be satisfied $\mathscr{B}_{i j}(E, X)$ would need to vanish as $1 / T$ passes through zero from negative to positive values. Aside from these considerations it is in any event clear that these results cannot be called in evidence for the preference for either form of the microcanonical entropy unless one wishes to apply the result to small systems, in which case the preference, at least in the case of the perfect fluid, would be for the bulk entropy formulation.

\section{The Zeroth Law}

The zeroth law of thermodynamics is an expression of the transitivity of thermal equilibrium between pairs of systems (see Sect. 5). As we showed in Sect. 12.1, if systems $\Xi_{\mathrm{A}}$ and $\Xi_{\mathrm{B}}$ are in thermal equilibrium then their bulk temperatures $T_{\Omega_{\mathrm{A}}}\left(E_{\mathrm{A}}, X_{\mathrm{A}}\right)$ and $T_{\Omega_{\mathrm{B}}}\left(E_{\mathrm{B}}, X_{\mathrm{B}}\right)$ have equal expectation values in the microcanonical distribution of the joined system (equation (12.3)). That this relation is satisfied by the bulk temperatures, and that the same relationship does not hold for the surface temperatures, is taken by Hilbert et al. (2014, Sect. VI.A.5) as an argument for choosing the bulk temperature over the surface temperature and consequently the bulk entropy over the surface entropy. However, as they themselves point out, and as we have shown in (12.5), for the surface temperature the appropriate relationship is in terms of inverse quantities. But, from the thermodynamic point of view, there is no reason why the equal-temperatures condition should be expressed in terms of temperatures rather than inverse temperatures. Indeed the fact that, in the thermodynamic context, where both positive and negative temperature are possible, the criterion (6.4) of relative hotness and coldness is expressed in terms of inverse temperatures would incline one to prefer the latter.

The downside to this analysis, both for bulk and surface temperatures is that the systems $\Xi_{\mathrm{A}}$ and $\Xi_{\mathrm{B}}$ remain thermally joined. If we wish to attribute the equivalence relation $\succ^{\mathrm{T}}$ to unjoined systems, then the picture, in either actuality or potentiality, is that of them being joined and subsequently split. When $\mathrm{S}(+)$ applies, the criterion for determining thermal equilibrium for $E_{\mathrm{A}}$ and $E_{\mathrm{B}}$ with $E_{\mathrm{A}}+E_{\mathrm{B}}=E_{\mathrm{T}}$ is the maximization of (5.3). If the bulk entropy is used this is equivalent to maximizing $\Omega_{\mathrm{A}}\left(E_{\mathrm{A}}, X_{\mathrm{A}}\right) \Omega_{\mathrm{B}}\left(E_{\mathrm{T}}-E_{\mathrm{A}}, X_{\mathrm{B}}\right)$ yielding the condition $T_{\Omega \mathrm{A}}\left(X_{\mathrm{A}}\right)=T_{\Omega \mathrm{B}}\left(X_{\mathrm{B}}\right)$. On the other hand, if the surface entropy is used, this is equivalent to maximizing $\omega_{\mathrm{A}}\left(E_{\mathrm{A}}, X_{\mathrm{A}}\right) \omega_{\mathrm{B}}\left(E_{\mathrm{T}}-E_{\mathrm{A}}, X_{\mathrm{B}}\right)$ yielding the condition $T_{\omega \mathrm{A}}\left(X_{\mathrm{A}}\right)=T_{\omega \mathrm{B}}\left(X_{\mathrm{B}}\right)$. Both choices of entropy satisfy the equal-temperatures condition. This argument can also be used in the $\mathrm{S}(-)$ case where the temperature conditions corresponds to minimization. However, from (12.1), in the $\mathrm{S}(+)$ case $T_{\omega \mathrm{A}}\left(X_{\mathrm{A}}\right)=T_{\omega \mathrm{B}}\left(X_{\mathrm{B}}\right)$ also corresponds to the energies of $\Xi_{\mathrm{A}}$ and $\Xi_{\mathrm{B}}$ taking their most probable values, which is the argument adopted by Frenkel and Warren (2015), thus reinforcing the case for surface rather than bulk quantities.

\section{The Second Law}

Hilbert et al. (2014) contend (Sect. V.B) that the bulk entropy satisfies the "Planck version of the second law", but (Sects. V.C and VI. C1) that it is violated by the surface entropy. This is a reference to the version of the second law given by Planck $\left(1903\right.$, Sect.133) ${ }^{66}$ and not the earlier version given by him in Sect. 116 and quoted in the footnote on page 18. We now explore the situation beginning first with a brief summary of the common ground. In doing so we confine attention to systems satisfying $\mathrm{S}(+)$, keeping open the question as to whether $\mathrm{U}(+)$ or $\mathrm{U}(-)$ (equivalently $\mathrm{T}(+)$ or $\mathrm{T}(-))$ is satisfied, except in so far as the two systems involved are of the same type.

\footnotetext{
66 "Every physical or chemical process in nature takes place in such a way as to increase the sum of the entropies of all the bodies taking any part in the process. In the limit, i.e. for reversible processes, the sum of the entropies remains unchanged."
} 
The subject of interest is two systems $\Xi_{\mathrm{A}}$ and $\Xi_{\mathrm{B}}$, which are thermally joined and then thermally split. As described in Sect. 5 from a purely thermodynamic point of view, if, with state $\left(\left(E, X_{\mathrm{A}}\right)\right) \in \Xi_{\mathrm{A}}$ and $\left(\left(E_{\mathrm{T}}-E, X_{\mathrm{B}}\right)\right) \in \Xi_{\mathrm{B}}$ the systems are thermally joined, an adiabatic process takes place resulting in the system $\Xi_{\mathrm{AB}}$ in state $\left(\left(E_{\mathrm{T}}, X_{\mathrm{A}}, X_{\mathrm{B}}\right)\right) \in \Xi_{\mathrm{A}}$ satisfying, from (5.1),

$$
S_{\mathrm{A}}\left(E, X_{\mathrm{A}}\right)+S_{\mathrm{B}}\left(E_{\mathrm{T}}-E, X_{\mathrm{B}}\right) \leq S_{\mathrm{AB}}\left(E_{\mathrm{T}}, X_{\mathrm{A}}, X_{\mathrm{B}}\right)
$$

The increase in entropy is accompanied by a flow of energy in the form of heat between the subsystems until they would be, if split, in thermal equilibrium with each other. That is until $E=\widetilde{E}$ where

$$
T_{\mathrm{A}}\left(\widetilde{E}, X_{\mathrm{A}}\right)=T_{\mathrm{B}}\left(E_{\mathrm{T}}-\widetilde{E}, X_{\mathrm{B}}\right) .
$$

The splitting which then occurs yields no further increase in entropy. So

$$
S_{\mathrm{AB}}\left(E_{\mathrm{T}}, X_{\mathrm{A}}, X_{\mathrm{B}}\right)=S_{\mathrm{A}}\left(\widetilde{E}, X_{\mathrm{A}}\right)+S_{\mathrm{B}}\left(E_{\mathrm{T}}-\widetilde{E}, X_{\mathrm{B}}\right)
$$

If $E=\widetilde{E}$ initially then there would be no increase in entropy during the joining process. That is to say, equality would apply in (16.1) if its left-hand side were at its maximum value with the two systems in thermal equilibrium prior to being joined.

There is a difference when these processes are viewed from a statistical mechanical viewpoint. When $\Xi_{\mathrm{A}}$ and $\Xi_{\mathrm{b}}$ are joined to form $\Xi_{A B}$ there is a flow of energy between the joined parts of $\Xi_{A B}$. But this does not cease when equilibrium is achieved, but continues in the form of fluctuations which would also be present if $\Xi_{\mathrm{A}}$ and $\Xi_{\mathrm{B}}$ were in equilibrium, that is in a state of maximum joint entropy, when they were joined. Such fluctuations away from the maximum entropy could be viewed as a violation of the second law. However, as we shall see, they are approximately normally distributed with standard deviation of $\mathrm{O}(1 / \sqrt{\aleph})$, making them in practise undetectable for large systems and vanishing in the thermodynamic limit. This picture is supported by the experimental work of Wang et al. (2002) who detected such violations of the second law for small systems over short periods of time. It is also reflected in the calculations of Lavis $(2005,2008)$ who showed, for various toy models, that, starting in an arbitrary state, the Boltzmann entropy, approached it maximum value from which it fluctuated with frequent small (downward) fluctuations and occasional larger fluctuations.

\section{- The Surface Entropy}

The case for the surface entropy is predicated on two assumptions:

(a) That $\eta \ll E$. This is inherent in the way that many authors define the microcanonical distribution with $\eta:=\triangle E$, the thickness of an energy shell. If this distribution is intended to correspond to a thermally isolated system with energy $E$, then the energy shell can be regarded as modelling a tolerance in observation; but only if the thickness of the shell is small relative to $E$.

(b) That the systems are large, in the sense described in Sect. 9, where the argument for restricting attention to large systems is made.

We now show that these are sufficient conditions for the validity of the surface entropy. That they are not also necessary, in the sense that (16.1) can hold where the relative sizes of $\eta$ and $E$ are more modestly separated is shown in the example provided by Hilbert et al. (op. cit.) and given below.

From (11.1) and (C.6), ${ }^{67}$

$$
S_{\omega \mathrm{AB}}\left(E_{\mathrm{T}}, X_{\mathrm{A}}, X_{\mathrm{B}} ; \eta\right)=\ln \left\{\int_{0}^{E_{\mathrm{T}}} \mathrm{d} E \exp \left[S_{\omega \mathrm{A}}\left(E, X_{\mathrm{A}} ; \eta\right)+S_{\omega \mathrm{B}}\left(E_{\mathrm{T}}-E, X_{\mathrm{B}} ; \eta\right)\right]\right\}-\ln (\eta) .
$$

The argument of the exponential has a maximum at $E=\widetilde{E}$, given by

$$
T_{\omega \mathrm{A}}\left(\widetilde{E}, X_{\mathrm{A}}\right)=T_{\omega \mathrm{B}}\left(E_{\mathrm{T}}-\widetilde{E}, X_{\mathrm{B}}\right),
$$

\footnotetext{
${ }^{67}$ It does not affect the argument in any way to choose the same $\eta$ for $\Xi_{\mathrm{A}}, \Xi_{\mathrm{B}}$ and $\Xi_{\mathrm{AB}}$.
} 
with

$$
\begin{gathered}
S_{\omega \mathrm{A}}\left(E, X_{\mathrm{A}} ; \eta\right)+S_{\omega \mathrm{B}}\left(E_{\mathrm{T}}-E, X_{\mathrm{B}} ; \eta\right)=S_{\omega \mathrm{A}}\left(\widetilde{E}, X_{\mathrm{A}} ; \eta\right)+S_{\omega \mathrm{B}}\left(E_{\mathrm{T}}-\widetilde{E}, X_{\mathrm{B}} ; \eta\right) \\
-\frac{1}{2\left[\sigma\left(\widetilde{E}, E_{\mathrm{T}}\right)\right]^{2}}\left(\frac{E}{\widetilde{E}}-1\right)^{2}+\mathrm{O}\left(\left[\frac{E}{\widetilde{E}}-1\right]^{3}\right),
\end{gathered}
$$

where

$$
\sigma\left(\widetilde{E}, E_{\mathrm{T}}\right):=\frac{T_{\omega \mathrm{A}}(\widetilde{E}, X)}{\widetilde{E}} \sqrt{\frac{C_{\omega \mathrm{A}}\left(\widetilde{E}, X_{\mathrm{A}}\right) C_{\omega \mathrm{B}}\left(E_{\mathrm{T}}-\widetilde{E}, X_{\mathrm{B}}\right)}{C_{\omega \mathrm{A}}\left(\widetilde{E}, X_{\mathrm{A}}\right)+C_{\omega \mathrm{B}}\left(E_{\mathrm{T}}-\widetilde{E}, X_{\mathrm{B}}\right)}} .
$$

In terms of the variable $E / \widetilde{E}$ and to second degree the fluctuations are normally distributed with unit mean and standard deviation $\sigma\left(\widetilde{E}, E_{\mathrm{T}}\right)$ of $\mathrm{O}(1 / \sqrt{\aleph})$. In the large system limit the distribution is steep about its mean allowing the integral to be evaluated and substituting into $(16.4)^{68}$

$$
\begin{aligned}
S_{\omega \mathrm{AB}}\left(E_{\mathrm{T}}, X_{\mathrm{A}}, X_{\mathrm{B}} ; \eta\right) \simeq & -\ln \left(\frac{\eta}{E_{0}}\right)+S_{\omega \mathrm{A}}\left(\widetilde{E}, X_{\mathrm{A}} ; \eta\right)+S_{\omega \mathrm{B}}\left(E_{\mathrm{T}}-\widetilde{E}, X_{\mathrm{B}} ; \eta\right) \\
& +\frac{1}{2} \ln \left\{\frac{2 \pi\left[T_{\omega \mathrm{A}}(\widetilde{E}, X)\right]^{2} C_{\omega \mathrm{A}}\left(\widetilde{E}, X_{\mathrm{A}}\right) C_{\omega \mathrm{B}}\left(E_{\mathrm{T}}-\widetilde{E}, X_{\mathrm{B}}\right)}{E_{0}^{2}\left[C_{\omega \mathrm{A}}\left(\widetilde{E}, X_{\mathrm{A}}\right)+C_{\omega \mathrm{B}}\left(E_{\mathrm{T}}-\widetilde{E}, X_{\mathrm{B}}\right)\right]}\right\},
\end{aligned}
$$

where, to make the arguments of the logarithms dimensionless $E_{0}$ is the unit energy. Even if, as in the example below, $\eta$ were $\mathrm{O}(\boldsymbol{\aleph})$ it would still be the case that

$$
S_{\omega \mathrm{AB}}\left(E_{\mathrm{T}}, X_{\mathrm{A}}, X_{\mathrm{B}} ; \eta\right) \stackrel{\mathrm{TL}}{\simeq} S_{\omega \mathrm{A}}\left(\widetilde{E}, X_{\mathrm{A}} ; \eta\right)+S_{\omega \mathrm{B}}\left(E_{\mathrm{T}}-\widetilde{E}, X_{\mathrm{B}} ; \eta\right) .
$$

We have, however, argued that $\eta$ should be small; in particular from (16.8) if $\eta \leq E_{0}$

$$
S_{\omega \mathrm{AB}}\left(E_{\mathrm{T}}, X_{\mathrm{A}}, X_{\mathrm{B}} ; \eta\right)>S_{\omega \mathrm{A}}\left(\widetilde{E}, X_{\mathrm{A}} ; \eta\right)+S_{\omega \mathrm{B}}\left(E_{\mathrm{T}}-\widetilde{E}, X_{\mathrm{B}} ; \eta\right) \geq S_{\omega \mathrm{A}}\left(E, X_{\mathrm{A}} ; \eta\right)+S_{\omega \mathrm{B}}\left(E_{\mathrm{T}}-E, X_{\mathrm{B}} ; \eta\right),
$$

with the first inequality replaced by equality only in the thermodynamic limit. As with the thermodynamic results (16.1)-(16.3), the surface entropy results (16.5), (16.9) and (16.10) apply both to positive and negative temperatures.

In opposition to this account Hilbert et al.(op. cit. Sect. V.C) assert that "there is no strict relation between the [surface] entropy of the compound system and the [surface] entropies of the subsystems before contact" because we have no information about the relative sizes of the two terms on the right-hand side of (16.4), meaning in particular the size of the constant $\eta$ (for which they use the symbol $\varepsilon$ ). They illustrate this point in Sect. VI.V by an explicit calculation for the case where, adapting to our notation slightly and dropping dependence on a deformation variable $X, \Xi_{\mathrm{A}}$ and $\Xi_{\mathrm{B}}$ are identical systems with

$$
\omega(E):= \begin{cases}\left(\frac{6 \Omega_{\mathrm{T}}}{E_{\mathrm{T}}^{3}}\right) E\left(E_{\mathrm{T}}-E\right), & 0 \leq E \leq E_{\mathrm{T}} \\ 0, & \text { otherwise }\end{cases}
$$

Substituting into (16.4)

$$
S_{\omega \mathrm{AB}}\left(E_{\mathrm{T}} ; \eta\right)=\ln \left(\frac{6 \eta \Omega_{\mathrm{T}}^{2}}{5 E_{\mathrm{T}}}\right)
$$

and, since by symmetry $\widetilde{E}=\frac{1}{2} E_{\mathrm{T}}$,

$$
S_{\omega \mathrm{A}}(\widetilde{E} ; \eta)+S_{\omega \mathrm{B}}\left(E_{\mathrm{T}}-\widetilde{E} ; \eta\right)=\ln \left(\frac{9 \eta^{2} \Omega_{\mathrm{T}}^{2}}{4 E_{\mathrm{T}}^{2}}\right) \geq S_{\omega \mathrm{A}}(E ; \eta)+S_{\omega \mathrm{B}}\left(E_{\mathrm{T}}-E ; \eta\right),
$$

\footnotetext{
${ }^{68}$ This is, of course, just the same as the application of Laplace's method, as used in Sect. 13.1.
} 
with the conclusion that (16.1) is not satisfied if $\eta>8 E_{\mathrm{T}} / 15$. Hilbert et al. infer from this, that the "only way way to avoid this problem in general is to always use an infinitesimal $[\eta]$ and a DoS that exactly gives the number of states at the energy in question (i.e. the exact degeneracy) devoid of any energy coarse-graining,"

It would seem to us that the inference Hilbert et al. draw from this example is well in excess of that which can be made from the calculations. The condition $\eta<8 E_{\mathrm{T}} / 15$ does not place very severe conditions on either the smallness of $\eta$ or the largeness of $E_{\mathrm{T}}$; much less than those imposed by(a) and (b) above.

\section{- The Bulk Entropy}

The case made by Hilbert et al. for the bulk entropy rests on the 'proof' in Sect. V.B that

$$
\Omega_{\mathrm{A}}\left(E_{\mathrm{A}}, X_{\mathrm{A}}\right) \Omega_{\mathrm{B}}\left(E_{\mathrm{B}}, X_{\mathrm{B}}\right) \leq \Omega_{\mathrm{AB}}\left(E_{\mathrm{A}}+E_{\mathrm{B}}, X_{\mathrm{A}}, X_{\mathrm{B}}\right),
$$

(their equation (48)). However, as has been pointed out by Swendsen and Wang (2016, Sect. VI.3), and as is clear from our derivation of (C.9), strict inequality applies in this relationship yielding

$$
S_{\Omega \mathrm{A}}\left(E, X_{\mathrm{A}}\right)+S_{\Omega \mathrm{B}}\left(E_{\mathrm{T}}-E, X_{\mathrm{B}}\right)<S_{\Omega \mathrm{AB}}\left(E_{\mathrm{T}}, X_{\mathrm{A}}, X_{\mathrm{B}}\right),
$$

which, as we have shown above, agrees with the relationship for the surface entropy for systems which are large but not at the thermodynamic limit. Thus the case where equality applies is left unresolved for the bulk entropies. For a power-law density of states this gap is closed by Hilbert et al. (2014, Sect. VIA3), who show equality in the thermodynamic limit. However, a rather more general approach mirroring the development in (16.4)-(16.9) can be used beginning with (C.8) rather than (C.6). Then we have

$$
S_{\Omega \mathrm{AB}}\left(E_{\mathrm{T}}, X_{\mathrm{A}}, X_{\mathrm{B}}\right)=\ln \left\{\int_{0}^{E_{\mathrm{T}}} \mathrm{d} E \exp \left[S_{\omega \mathrm{A}}\left(E, X_{\mathrm{A}} ; 1\right)+S_{\Omega \mathrm{B}}\left(E_{\mathrm{T}}-E, X_{\mathrm{B}}\right)\right]\right\} .
$$

And from (11.6), (13.7) and (13.8),

$$
\begin{aligned}
S_{\Omega \mathrm{AB}}\left(E_{\mathrm{T}}, X_{\mathrm{A}}, X_{\mathrm{B}}\right) & \stackrel{\mathrm{TL}}{\simeq} S_{\omega \mathrm{A}}\left(\stackrel{\circ}{E}, X_{\mathrm{A}} ; 1\right)+S_{\Omega \mathrm{B}}\left(E_{\mathrm{T}}-\stackrel{\circ}{E}, X_{\mathrm{B}}\right) \stackrel{\mathrm{TL}}{\simeq} S_{\Omega \mathrm{A}}\left(\stackrel{\circ}{E}, X_{\mathrm{A}}\right)+S_{\Omega \mathrm{B}}\left(E_{\mathrm{T}}-\stackrel{\circ}{E}, X_{\mathrm{B}}\right), \\
S_{\Omega \mathrm{AB}}\left(E_{\mathrm{T}}, X_{\mathrm{A}}, X_{\mathrm{B}}\right) & >S_{\omega \mathrm{A}}\left(\stackrel{\circ}{E}, X_{\mathrm{A}} ; 1\right)+S_{\Omega \mathrm{B}}\left(E_{\mathrm{T}}-\stackrel{\circ}{E}, X_{\mathrm{B}}\right) \\
& \stackrel{\mathrm{TL}}{\simeq} S_{\Omega \mathrm{A}}\left(\stackrel{\circ}{E}, X_{\mathrm{A}}\right)+S_{\Omega \mathrm{B}}\left(E_{\mathrm{T}}-\stackrel{\circ}{E}, X_{\mathrm{B}}\right) \\
& \geq S_{\Omega \mathrm{A}}\left(E, X_{\mathrm{A}}\right)+S_{\Omega \mathrm{B}}\left(E_{\mathrm{T}}-E, X_{\mathrm{B}}\right),
\end{aligned}
$$

where

$$
T_{\Omega \mathrm{B}}\left(E_{\mathrm{T}}-\stackrel{\circ}{E}, X_{\mathrm{B}}\right)=T_{\omega \mathrm{A}}\left(\stackrel{\circ}{E}, X_{\mathrm{A}}\right) \stackrel{\mathrm{TL}}{\simeq} T_{\Omega \mathrm{A}}\left(\stackrel{\circ}{E}, X_{\mathrm{A}}\right) .
$$

So, by a slightly less straightforward calculation, the bulk entropy can be shown to satisfy the same large-system thermodynamic-limit results as the surface entropy. However, of course, this applies only to positive temperatures, although, unlike the case of the surface entropy, the inequality (16.15) is not restricted to large systems.

\section{The Spin- $\frac{1}{2}$ Example of Campisi (2015)}

The spin model introduced in Sect. D of Campisi's paper with Hamiltonian ${ }^{69}$

$$
\mathcal{H}_{\mathrm{CP}}:=-B \mu\left(n_{\uparrow}-n_{\downarrow}\right),
$$

\footnotetext{
${ }^{69}$ We distinguish variables which he employs with a different meaning from ours by using a subscript 'CP' and enclose his equation numbers in square brackets
} 
where $\mu$ is the magnetic moment of the spins and $B$ is a magnetic field, is mathematically equivalent to $\mathfrak{B}_{2}$, the discrete energy system discussed in Sects. 10.2, 11.2 and 13.2 with energy levels $\{0, \varepsilon\}$. However, from a statistical mechanical point of view there is an important difference. In the $\mathfrak{B}_{2}$ model $\varepsilon$ in (10.6) is an internal parameter, and, although we used the micromechanical and Gibbs distributions, there was in fact no controllable intensive mechanical variable, whereas in (17.1) the role of $\varepsilon$ is played by $-2 B \mu$, where $B$ is a controllable intensive mechanical variable.

Rather confusingly Campisi relates his Hamiltonian $\mathcal{H}_{\mathrm{CP}}$ to his energy $E_{\mathrm{CP}}$ using an expectation, that is $\left\langle\mathcal{H}_{\mathrm{CP}}\right\rangle=E_{\mathrm{CP}}$. However, of course, the substance of the discussion uses the micromechanical distribution, where the mechanical energy is an independent variable equal to the value of the Hamiltonian which is fixed without need for expectations. To simplify the discussion we shall, then, just refer to the energies, which for the present work are one variable $E$ and two parameters $\eta$ and $\varepsilon$, and for Campisi are two variables $E_{\mathrm{CP}}$ and $B$, and one parameter $\varepsilon_{\mathrm{CP}}$. Given that the up-spin state is identified with the energy level $\varepsilon>0$ so that $X=n_{1}=n_{\uparrow}$ and $B<0,{ }^{70}$

$$
E=\varepsilon X \equiv-2 B \mu n_{\uparrow}, \quad \varepsilon \equiv-2 B \mu, \quad E \equiv E_{\mathrm{CP}}-B \mu N, \quad X \equiv \frac{N}{2}-\frac{E_{\mathrm{CP}}}{2 B \mu} .
$$

In the $\mathfrak{B}_{m}$ model the entropy is a function of $E$ through its dependence on $X=E / \varepsilon$; so

$$
\frac{1}{T}=\frac{\partial S(X)}{\partial E}=\frac{1}{\varepsilon} \frac{\partial S(X)}{\partial X} .
$$

In Campisi's model the entropy is a function of $E_{\mathrm{CP}}$ and $B$ through its dependence on $X$; so

$$
\frac{1}{T_{\mathrm{CP}}}=\frac{\partial S(X)}{\partial E_{\mathrm{CP}}}=-\frac{1}{2 B \mu} \frac{\partial S(X)}{\partial X}, \quad \frac{\mathfrak{M}}{T_{\mathrm{CP}}}=\frac{\partial S(X)}{\partial B}=\frac{E_{\mathrm{CP}}}{2 B^{2} \mu} \frac{\partial S(X)}{\partial X},
$$

where $\mathfrak{M}$ is the magnetization, which from (17.4) satisfies

$$
\mathfrak{M}=-E_{\mathrm{CP}} / B,
$$

which he otherwise shows is the correct relationship between magnetization, energy and magnetic field. Campisi derives (17.4) and (17.5) (respectively [32] and [33]) for the bulk entropy, but not by the surface entropy. The root of the problem is in the way that the surface entropy parameter, which we denoted as $\eta$ and which Campisi in [1] denotes as $\varepsilon$ (and which we for the sake of clarity denote as $\varepsilon_{\mathrm{CP}}$ ) is handled. In $\mathfrak{B}_{2}$, the density of energy states is the number of energy states per unit of $\varepsilon$ giving (10.9) and a similar relationship is given in Campisi's equation [12], where $2 \mu|B|$ is used as the divisor. However, the factor $\varepsilon_{\mathrm{CP}}$ in [1] is left intact yielding

$$
S_{\omega}\left(E_{\mathrm{CP}}, B\right)=S_{\omega}^{\prime}(X)+\ln \left(\varepsilon_{\mathrm{CP}} / 2 \mu|B|\right)
$$

and hence his [33]

$$
\mathfrak{M}=-\frac{E_{\mathrm{CP}}+T_{\mathrm{CP}}}{B} \text {. }
$$

In Sect. 11.2 we have argued for the identification $\eta:=\varepsilon$ in the $\mathfrak{B}_{m}$ model both by comparison with the continuous energy case and by reference to Boltzmann's original form for his entropy. This identification in the form $\varepsilon_{\mathrm{CP}}:=2 \mu|B|$ for Campisi's model resolves the problem exposed in (17.6). This means that the magnetic field here has a dual role. On the one hand it is the mechanical intensive controllable variable and on the other it fixes the spacing of the energy states. This is an artifact of this particularly simple model which would not be present in, for example, the Ising model, where the change in energy due to a single spin flip would contain more that just a field-dependent contribution.

An alternative way to solve the problem is that adopted by Abrahm and Penrose (2017). They effectively take $\eta:=1$ so that the multiplication by $\eta$ and subsequent division leading to (11.23) would not occur. Equivalently in the spin model $\varepsilon_{\mathrm{CP}}:=1$ and there is no division by $2 \mu|B|$ in [29]. The rationale for this approach could be based simply on the reason for introducing a constant of dimension $\mathbf{J}$ into the surface entropy. For a continuous-energy system the density of states is of dimension $\mathrm{J}^{-1}$ and an energy-dimensioned quantity is needed to make the argument of the logarithm dimensionless. However, for a discrete-energy system the density of states is just a dimensionless counting of states and such a constant is unnecessary. Whichever of these arguments is used the effect as Abrahm and Penrose comment is to make this spin example "neutral between the "Gibbs" [(bulk)] and "Boltzmann" [(surface)] entropies."

\footnotetext{
${ }^{70} \mathrm{~A}$ similar mapping between the models applies when $B>0$.
} 


\section{Thermodynamic Consistency}

In this case we consider a system where $\boldsymbol{x}:=(\boldsymbol{p}, \boldsymbol{q})$ with an evolution determined by Hamilton's equations (C.11). The relationship (C.18) is the condition for a function $W(E, X)$ to be an adiabatic invariant in the thermodynamic sense, which means that $W(E, X)=W(S(E, X))$. Substituting this form into (C.18), combining with (C.15) and using Table 3 gives

$$
\frac{\partial E(S, X)}{\partial X}=\xi(E, X)=-T \frac{\partial S(E, X)}{\partial X}=\left\langle\frac{\partial \mathcal{H}(\boldsymbol{p}, \boldsymbol{q} ; X)}{\partial X} \mid E, X\right\rangle
$$

Equality between the first three terms in this relationship follows directly from (8.1). So the interest is in equality between the third and fourth terms. This implies that $S(E, X)$ is an adiabatic invariant in the mechanical sense defined in Appen. C, where the analysis was predicated on $S(E, X)$ being an invertible function of $E$; that is on temperature having a single sign. This condition is satisfied by the bulk entropy with the bulk temperature being always positive and from (11.5), (11.6) and (11.15) it can be seen that the bulk functions satisfy the equality between the third and fourth terms of (18.1). That a similar result does not hold for the surface entropy is part of the argument advanced by Dunkel and Hilbert (2014a) for rejecting the surface entropy in favour of the bulk entropy. However, as will be clear from the following argument advanced by Frenkel and Warren (2015), ${ }^{71}$ the situation is rather less clear cut. From (12.10) and (12.16),

$$
\left\langle\frac{\partial \mathcal{H}(\boldsymbol{p}, \boldsymbol{q} ; X)}{\partial X} \mid T, X\right\rangle \exp (-A(T, X) / T)=\int_{0}^{E_{\max }}\left\langle\frac{\partial \mathcal{H}(\boldsymbol{p}, \boldsymbol{q} ; X)}{\partial X} \mid E, X\right\rangle \exp \left[-A_{\omega}(E ; T, X ; \eta) / T\right] \mathrm{d} E,
$$

where

$$
A_{\omega}(E ; T, X ; \eta):=E-S_{\omega}(E, X ; \eta) T
$$

It was showed in Sect. 13.1, that, if $S_{\omega}(E, X ; \eta)$ is a concave non-negative function of $E$, $\zeta_{\omega}(E, X ; \eta):=-A_{\omega}(E ; T, X ; \eta) / T=S_{\omega}(E, X ; \eta)-E / T$ has a steep maximum at

$$
E=E^{\star}(T, X) \quad \text { satisfying } \quad T_{\omega}\left(E^{\star}, X\right)=T .
$$

These conditions can be satisfied when $T>0$ and $S(E, X)$ is monotonically increasing for $E \in\left[0, E_{\max }\right]$ and when $T<0$ and $S(E, X)$ is monotonically decreasing for $E \in\left[0, E_{\max }\right]$. In the latter case the condition $S_{\omega}(0, X ; \eta)=0$, which we assumed in Sect. 13.1 must be violated. Whilst this does not necessarily exclude the consideration of negative temperatures ${ }^{72}$ our main aim is to compare the surface and bulk entropies and we now restrict attention to the case $T>0$ where, from (12.17) and (18.2)-(18.4),

$$
\begin{aligned}
\xi_{\mathrm{c}}(T, X)=\left\langle\frac{\partial \mathcal{H}(\boldsymbol{p}, \boldsymbol{q} ; X)}{\partial X} \mid T, X\right\rangle \stackrel{\mathrm{TL}}{\simeq}\left\langle\frac{\partial \mathcal{H}(\boldsymbol{p}, \boldsymbol{q} ; X)}{\partial X} \mid E^{\star}, X\right\rangle \\
\qquad\left\{\begin{array}{l}
\stackrel{\mathrm{TL}}{\simeq} \xi\left(E^{\star}, X\right)=-T_{\omega}\left(E^{\star}, X\right) \frac{\partial S_{\omega}\left(E^{\star}, X ; \eta\right)}{\partial X}=\xi_{\omega}\left(E^{\star}, X\right) \\
=\xi\left(E^{\star}, X\right)=-T_{\Omega}\left(E^{\star}, X\right) \frac{\partial S_{\Omega}\left(E^{\star}, X ; \eta\right)}{\partial X}=\xi_{\Omega}\left(E^{\star}, X\right)
\end{array}\right.
\end{aligned}
$$

The bulk entropy and temperature satisfy the consistency condition (18.1) exactly and the surface entropy and temperature satisfy it in the thermodynamic limit, corresponding to the pair of $\stackrel{\mathrm{TL}}{\simeq}$ and $=$ in (18.5) (a) and (b) respectively. However, of course, when the comparison is made with the canonical distribution both cases contain the relationship $\stackrel{\mathrm{TL}}{\simeq}$. This is to be expected since the intensive variables $\xi_{\mathrm{c}}(T, X), \xi_{\omega}(E, X)$ and $\xi_{\Omega}(E, X)$ are uncontrolled in their respective distributions, coinciding when $E=E^{\star}$ only in the thermodynamic limit when $E^{\star}=\langle E \mid T, X\rangle$.

\footnotetext{
${ }^{71}$ A rejoinder to this paper, which was first published on line in 2014, by Dunkel and Hilbert (2014b) does not seek to challenge this argument.

${ }^{72}$ In the case of an entropy profile like that shown in Fig. 6 the energy range can be restricted to that within subsystem $\Xi \llbracket[-+]$ for which $S(E, X)$ is invertible.
} 


\section{Conclusions}

It is commonly the case that both the internal energy and entropy of a system do not decrease during an adiabatic process. The situation where the internal energy decreases and the entropy increases is associated with negative temperatures and, as we have shown in Sect. 1.1, there is good evidence that this behaviour occurs in some real systems. Entropy decrease in adiabatic processes is associated with negative heat capacity at constant deformation variables and, as we have briefly indicated in the footnote on page 18, there is also evidence that this can occur albeit in rather exotic circumstances.

The account of thermodynamics by Lieb and Yngvason (1999) is based on a set of axioms and a definition of entropy, which we provide in Appen. A. Together these exclude the possibility of entropy decrease during adiabatic processes. However, the argument for excluding energy decrease, and thus negative temperatures, is informal and effectively consists of the choice, on physical grounds, of supposing that we live in a 'world' in which temperatures are always positive. In this work we are interested in the possibility of a system undergoing an adiabatic process between states of negative and positive temperature. This implies that the thermodynamic space of the system must be a union of subspaces with opposite signs of the temperature. We have shown how the Lieb and Yngvason formulation can be modified to accommodate such a decomposition.

The same decomposition scheme can also be applied to the approach based directly on Carathéodory's version of the second law, which allows both internal energy and entropy decrease in adiabatic processes. Given this rather general picture with both signs for the temperature and increasing and decreasing entropy in adiabatic processes it is of interest to explore the modifications needed to the Kelvin-Planck (heat-engine) and Clausius ( heat-pump) versions of the second law. By examining in detail the behaviour of a cyclic sequence of processes, we have shown that the Kelvin-Planck but not the Clausius version needs modification when negative temperatures are allowed. However, providing also for the possibility of entropy decrease involves modifications to both forms of the law.

In the second part of this paper we addressed the problem of the way that negative temperatures can be exhibited by statistical mechanical models. Work on this has largely concentrated on thermally isolated systems, narrowing to the rival claims of the surface and bulk forms for the entropy of a microcanonical or micromechanical system. This dispute is fundamental, since a range of thermodynamic behaviour with a negative temperature is possible using the surface entropy but not using the bulk entropy. A key to the resolution of this question lies in the equivalence of distributions in the thermodynamic limit. In this case the equivalence is between the microcanonical or micromechanical distribution on the one hand and the canonical or Gibbs distribution on the other. We have shown that both the surface and bulk entropies, and temperatures, satisfy this equivalence in the thermodynamic limit for positive temperatures. For negative temperatures equivalence continues to hold for the surface quantities, but fails for the bulk quantities, where the inverse bulk temperature is asymptotically zero. These results are supported by explicit calculations for the $\mathfrak{B}_{m}$ model. In Part III we have addressed the various claims that the surface entropy violates basic requirements of thermodynamics. Much of this discussion concentrates on systems satisfying Hamiltonian dynamics where the energy has no upper bound and the temperature is always positive. Here we do not dispute that the bulk entropy is thermodynamically consistent. What we have shown is that in all cases this is also true for the surface entropy in the thermodynamic limit.

\section{Appendices}

\section{A. Supporting Material for Sect. 4.1}

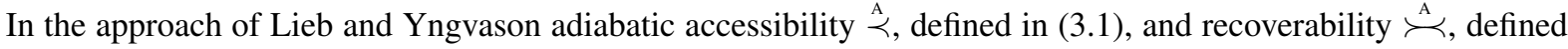
in (3.2), satisfy the following axioms:

Axiom A-I: Reflexivity: $X \stackrel{\mathrm{A}}{\prec} \boldsymbol{X}$.

Axiom A-II: Transitivity: $X \stackrel{\mathrm{A}}{\prec} \boldsymbol{Y} \wedge \boldsymbol{Y} \stackrel{\mathrm{A}}{\prec} \boldsymbol{Z} \Longrightarrow \boldsymbol{X} \stackrel{\mathrm{A}}{\prec} \boldsymbol{Z}$. 
Axiom A-III: Cartesian-Product Consistency: $\boldsymbol{X} \stackrel{\mathrm{A}}{\prec} \boldsymbol{Y} \wedge \boldsymbol{X}^{\prime} \stackrel{\mathrm{A}}{\prec} \boldsymbol{Y}^{\prime} \Longrightarrow\left(\boldsymbol{X}, \boldsymbol{X}^{\prime}\right) \stackrel{\mathrm{A}}{\prec}\left(\boldsymbol{Y}, \boldsymbol{Y}^{\prime}\right)$.

Axiom A-IV: Scaling Consistency: $\boldsymbol{X} \stackrel{\mathrm{A}}{\prec} \boldsymbol{Y} \Longrightarrow \lambda \boldsymbol{X} \stackrel{\mathrm{A}}{\prec} \lambda Y, \forall \lambda>0$.

Axiom A-V: Splitting and Recombination: $\Xi \ni \boldsymbol{X} \stackrel{\mathrm{A}}{\prec}([1-\lambda] \boldsymbol{X}, \lambda \boldsymbol{X}) \in \Xi(1-\lambda) \times \Xi(\lambda), \forall \boldsymbol{\lambda} \in(0,1)$.

Axiom A-VI: Stability: $\left(\boldsymbol{X}, \varepsilon \boldsymbol{X}^{\prime}\right) \stackrel{\mathrm{A}}{\prec}\left(\boldsymbol{Y}, \varepsilon \boldsymbol{Y}^{\prime}\right)$, for some $\boldsymbol{X}^{\prime}$ and $\boldsymbol{Y}^{\prime}$ and a sequence of values of $\varepsilon$ tending to zero, $\Longrightarrow \boldsymbol{X} \stackrel{\text { A }}{\prec} \boldsymbol{Y}$.

Axiom A-VII: Convex Combination: $\Xi(1-\lambda) \times \Xi(\lambda) \ni([1-\lambda] \boldsymbol{X}, \lambda \boldsymbol{Y}) \stackrel{\mathrm{A}}{\prec}[1-\lambda] \boldsymbol{X}+\lambda \boldsymbol{Y} \in \Xi, \forall \boldsymbol{X}, \boldsymbol{Y} \in \Xi$ and $\lambda \in(0,1)$.

Of these axioms all but A-V and A-VII involve adiabatic accessibility between states of a single system; they also exhibit a certain 'common-sense' quality in that if one were considering processes within a system or within Cartesian products or scaled copies of a system, this is how one would expect adiabatic accessibility to behave. The exceptions to this are Axioms A-V and A-VII, which both involve adiabatic accessibility between a system $\Xi$ and $\Xi(1-\lambda) \times \Xi(\lambda)$ for some $\lambda \in(0,1)$. They can be regarded as precursors of Lieb and Yngvason's thermal contact and thermal splitting axioms (T-I and T-II, op. cit., p. 55) and A-VII is of crucial importance in the establishment of the properties of forward sectors. In particular it is necessary to prove:

Theorem A.1. (op. cit, p. 45)

If, for $\boldsymbol{X}_{1}, \boldsymbol{X}_{2}, \boldsymbol{X}_{3} \in \Xi, \boldsymbol{X}_{2}:=[1-\lambda] \boldsymbol{X}_{1}+\lambda \boldsymbol{X}_{3}, \lambda \in(0,1)$, then $\boldsymbol{X}_{2} \stackrel{A}{\prec} \boldsymbol{X}_{3} \Longrightarrow \boldsymbol{X}_{1} \stackrel{A}{\prec} \boldsymbol{X}_{2}$.

which we use in the discussion in Sect. 4.1. It is also useful at this stage to introduce the following logical statements:

$\mathrm{ACP}(\Xi):=\left\{\forall \boldsymbol{X}, \boldsymbol{X}^{\prime} \in \Xi, \boldsymbol{X} \stackrel{\mathrm{A}}{\complement} \boldsymbol{X}^{\prime}\right\}:$ : the adiabatic comparability hypothesis,

$\mathrm{UAI}(\Xi):=\left\{\forall \boldsymbol{X} \in \Xi, \exists \boldsymbol{X}_{\mathrm{UX}} \in \Xi\right.$ such that $\left.\boldsymbol{X} \stackrel{\mathrm{A}}{\prec} \boldsymbol{X}_{\mathrm{UX}}\right\} \quad:$ the upper adiabatic irrecoverability hypothesis,

$\operatorname{LAI}(\Xi):=\left\{\forall \boldsymbol{X} \in \Xi, \exists \boldsymbol{X}_{\mathrm{LX}} \in \Xi\right.$ such that $\left.\boldsymbol{X}_{\mathrm{LX}} \stackrel{\mathrm{A}}{\prec} \boldsymbol{X}\right\} \quad:$ the lower adiabatic irrecoverability hypothesis,

where $\boldsymbol{X}_{\mathrm{UX}}$ and $\boldsymbol{X}_{\mathrm{LX}}$ are dependent of $\boldsymbol{X}$. The truth of both UAI( $\left.\Xi\right)$ and $\mathrm{LAI}(\boldsymbol{\Xi})$ are asserted by Lieb and Yngvason's thermal Axiom T-IV and it can also be shown (op. cit., Thm. 2.9) that UAI $(\Xi) \Longrightarrow$ Cth-I $(\Xi)$ with the converse holding if the interiors of all forward sectors $\mathscr{F}_{\mathbf{x}}^{\circ}$ defined in (A.4) are non-empty. The truth of the adiabatic comparability hypothesis $\operatorname{ACP}(\Xi)$ is closely related to the structure of forward sectors.

\section{A.1. Forward Sectors}

The set

$$
\begin{array}{ll}
\mathscr{F}_{\mathbf{X}}:=\left\{\boldsymbol{X}^{\prime} \mid \boldsymbol{X} \prec \boldsymbol{X}^{\prime}\right\}, & \text { is the forward sector of } \boldsymbol{X} . \\
\mathscr{F}_{\mathbf{X}}^{\circ}:=\left\{\boldsymbol{X}^{\prime} \in \mathscr{F}_{\mathbf{X}} \mid \exists \mathscr{N}_{\boldsymbol{X}^{\prime}} \subset \mathscr{F}_{\mathbf{X}}\right\} & \text { is the interior of } \mathscr{F}_{\mathbf{X}}, \\
\partial \mathscr{F}_{\mathbf{X}}:=\left\{\boldsymbol{X}^{\prime} \in \mathscr{F}_{\mathbf{X}} \mid \nexists \mathscr{N}_{\boldsymbol{X}^{\prime}} \subset \mathscr{F}_{\mathbf{X}}\right\} & \text { is the boundary of } \mathscr{F}_{\mathbf{X}},
\end{array}
$$

where $\mathscr{N}_{\mathbf{X}} \subset \Xi$ is an open neighbourhood of $\boldsymbol{X}$. And the following statements summarize the results of Lieb and Yngvason (1999) for forward sectors:

(i) From Axioms A-I to A-VII (and particularly from A-VII), $\mathscr{F}_{\mathbf{x}}$ is convex (op. cit., Thm. 2.6).

(ii) From $\operatorname{UAI}(\Xi), \partial \mathscr{F}_{\mathbf{X}}$ is a relatively ${ }^{73}$ closed set containing $\boldsymbol{X}$.

(iii) From $\operatorname{UAI}(\Xi)$ and the assumption that $\partial \mathscr{F}_{\mathbf{X}}$ has a unique tangent plane $\Phi_{\mathbf{X}}$ at $\boldsymbol{X}$ (Lieb and Yngvason's Axiom S-II), $\mathscr{F}_{\mathbf{x}}^{\circ} \neq \emptyset$ (op. cit., Thm. 3.2).

From (ii) and (iii), $\mathscr{F}_{\mathbf{X}}$ is a relatively closed proper subset of $\Xi$.

The set of forward sectors $\left\{\mathscr{F}_{\mathbf{X}} \mid \boldsymbol{X} \in \Xi\right\}$ is nested if $\forall \boldsymbol{X}, \boldsymbol{Y} \in \Xi$ exactly one of the following is satisfied: (a) $\mathscr{F}_{\boldsymbol{Y}}=\mathscr{F}_{\mathbf{X}}$, (b) $\mathscr{F}_{\boldsymbol{Y}} \subset \mathscr{F}_{\mathbf{X}}^{\circ}$, (c) $\mathscr{F}_{\mathbf{X}} \subset \mathscr{F}_{\boldsymbol{Y}}^{\circ}$.

\footnotetext{
${ }^{73}$ The need for relative closure is because part of the boundary of $\partial \mathscr{F} \mathbf{x}$ may not lie in $\Xi$.
} 


\section{A.2. Entropy}

As has already been indicated, important differences between the Carathéodory and Lieb and Yngvason approaches to thermodynamics are that the former establishes the existence of the entropy function with the appropriate properties including the possibility that either $\mathrm{S}(+)$ or $\mathrm{S}(-)$ is true and the latter provides an explicit definition of entropy which applies only to the case where $\mathrm{S}(+)$ is true. Here we provide a brief summary of the Lieb and Yngvason account in which they showed that there is an entropy function $S$, which is unique to within an affine transformation, ${ }^{74}$ over a family of systems closed under scaling and Cartesian product formation and which satisfies:

- For $\boldsymbol{X}, \boldsymbol{Y} \in \Xi$,

$$
\begin{array}{lll}
\boldsymbol{X} \stackrel{\mathrm{A}}{\prec} \boldsymbol{Y} & \Longleftrightarrow \quad S(\boldsymbol{X})<S(\boldsymbol{Y}), \\
\boldsymbol{X} \stackrel{\mathrm{A}}{\prec} \boldsymbol{Y} \quad \Longleftrightarrow \quad S(\boldsymbol{X})=S(\boldsymbol{Y}) .
\end{array}
$$

- For $\boldsymbol{X} \in \Xi$ and $\lambda \boldsymbol{X} \in \Xi(\lambda)$,

$$
S(\lambda \boldsymbol{X})=\lambda S(\boldsymbol{X}) .
$$

- For $\boldsymbol{X}_{1} \in \Xi_{1}, \boldsymbol{X}_{2} \in \Xi_{2}$ and $\left(\boldsymbol{X}_{1}, \boldsymbol{X}_{2}\right) \in \Xi_{1} \times \Xi_{2}$

$$
S\left(\left(\boldsymbol{X}_{1}, \boldsymbol{X}_{2}\right)\right)=S\left(\boldsymbol{X}_{1}\right)+S\left(\boldsymbol{X}_{2}\right) .
$$

The development has two stages. The first is to establish a local entropy function $S_{\Xi}$ for any system $\Xi$, which is

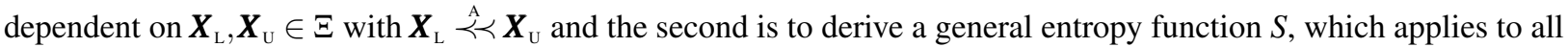
the systems of the family with in each case just one choice of a state where the entropy is zero. In the first stage it is shown that:

(i) From $\operatorname{ACP}(\Xi), \operatorname{UAl}(\Xi)$ and $\operatorname{LAl}(\Xi), \exists \boldsymbol{X} \in \Xi$ with $\boldsymbol{X}_{\mathrm{L}} \stackrel{\mathrm{A}}{\prec} \boldsymbol{X} \stackrel{\mathrm{A}}{\gtrless} \boldsymbol{X}_{\mathrm{U}}$.

(ii) From UAI( $\Xi)$ and A-VII

$$
\Sigma_{\lambda}\left(\boldsymbol{X}_{\mathrm{L}}, \boldsymbol{X}_{\mathrm{U}}\right):=\left\{\boldsymbol{X} \in \Xi \mid\left([1-\lambda] \boldsymbol{X}_{\mathrm{L}}, \lambda \boldsymbol{X}_{\mathrm{U}}\right) \stackrel{A}{\prec} \boldsymbol{X}\right\} \subseteq \Xi,
$$

is a convex set.

(iii) Since $\forall \boldsymbol{X}, \boldsymbol{Y} \in \Xi$ with $\boldsymbol{X} \stackrel{\text { R }}{\gtrless} \boldsymbol{Y}$

$$
([1-\lambda] \boldsymbol{X}, \lambda \boldsymbol{Y}) \stackrel{\mathrm{A}}{\prec}([1-\mu] \boldsymbol{X}, \mu \boldsymbol{Y}) \Longleftrightarrow \lambda \leq \mu,
$$

(Lieb and Yngvason, 1999, Lemma 2.2),

$$
\Sigma_{\mu}\left(\boldsymbol{X}_{\mathrm{L}}, \boldsymbol{X}_{\mathrm{U}}\right) \subseteq \Sigma_{\lambda}\left(\boldsymbol{X}_{\mathrm{L}}, \boldsymbol{X}_{\mathrm{U}}\right) \quad \Longleftrightarrow \lambda \leq \mu .
$$

(iv) It follows from (A.10) that, for fixed $\boldsymbol{X}_{\mathrm{L}}, \boldsymbol{X}_{\mathrm{U}} \in \Xi$, there exists a maximum $\lambda$ such that $\boldsymbol{X} \in \Sigma_{\lambda}\left(\boldsymbol{X}_{\mathrm{L}}, \boldsymbol{X}_{\mathrm{U}}\right)$ and

$$
S_{\Xi}\left(\boldsymbol{X} \mid \boldsymbol{X}_{\mathrm{L}}, \boldsymbol{X}_{\mathrm{U}}\right):=\sup \left\{\boldsymbol{\lambda} \mid \boldsymbol{X} \in \Sigma_{\lambda}\left(\boldsymbol{X}_{\mathrm{L}}, \boldsymbol{X}_{\mathrm{U}}\right)\right\}
$$

is the definition of local entropy (Lieb and Yngvason, 1999, p. 26).

\footnotetext{
${ }^{74}$ Two function which are equal to within an affine transformation are said to be WAT-equal. If all functions satisfying particular conditions are WAT-equal, then any one of these functions satisfying the conditions is said to be WAT-unique with respect to these conditions.
} 
(v) Given $\operatorname{ACP}(\Xi(1-\lambda) \times \Xi(\lambda))$ and $\operatorname{UAI}(\Xi), S_{\Xi}\left(\boldsymbol{X} \mid \boldsymbol{X}_{\mathrm{L}}, \boldsymbol{X}_{\mathrm{U}}\right)$ exists and is finite with

$$
\begin{aligned}
& \lambda=S_{\Xi}\left(\boldsymbol{X} \mid \boldsymbol{X}_{\mathrm{L}}, \boldsymbol{X}_{\mathrm{U}}\right) \Longleftrightarrow \\
& \boldsymbol{X} \stackrel{\mathrm{A}}{\prec}\left([1-\lambda] \boldsymbol{X}_{\mathrm{L}}, \lambda \boldsymbol{X}_{\mathrm{U}}\right),
\end{aligned}
$$

(Lieb and Yngvason, 1999, Lemma 2.1 and 2.3).

(vi) For $\boldsymbol{X}, \boldsymbol{X}^{\prime}, \boldsymbol{Y}, \boldsymbol{Y}^{\prime} \in \Xi$,

$$
\begin{aligned}
& \left([1-\mu] \boldsymbol{X}, \mu \boldsymbol{X}^{\prime}\right) \stackrel{\mathrm{A}}{\prec}\left(\left[1-\mu^{\prime}\right] \boldsymbol{Y}, \mu^{\prime} \boldsymbol{Y}^{\prime}\right) \Longleftrightarrow \\
& {[1-\mu] S_{\Xi}\left(\boldsymbol{X} \mid \boldsymbol{X}_{\mathrm{L}}, \boldsymbol{X}_{\mathrm{U}}\right)+\mu S_{\Xi}\left(\boldsymbol{X}^{\prime} \mid \boldsymbol{X}_{\mathrm{L}}, \boldsymbol{X}_{\mathrm{U}}\right) \leq\left[1-\mu^{\prime}\right] S_{\Xi}\left(\boldsymbol{Y} \mid \boldsymbol{X}_{\mathrm{L}}, \boldsymbol{X}_{\mathrm{U}}\right)+\mu^{\prime} S_{\Xi}\left(\boldsymbol{Y}^{\prime} \mid \boldsymbol{X}_{\mathrm{L}}, \boldsymbol{X}_{\mathrm{U}}\right),}
\end{aligned}
$$

(Lieb and Yngvason, 1999, Thm. 2.2), which generalizes (A.5) for the local entropy.

(vii) The local entropy is the WAT-unique function satisfying (A.14) (Lieb and Yngvason, 1999, Thm. 2.3).

(viii) For $\boldsymbol{X}, \boldsymbol{X}^{\prime} \in \Xi$, from A-VII,

$$
(1-\mu) S_{\Xi}\left(\boldsymbol{X} \mid \boldsymbol{X}_{\mathrm{L}}, \boldsymbol{X}_{\mathrm{U}}\right)+\mu S_{\Xi}\left(\boldsymbol{X}^{\prime} \mid \boldsymbol{X}_{\mathrm{L}}, \boldsymbol{X}_{\mathrm{U}}\right) \leq S_{\Xi}\left([1-\mu] \boldsymbol{X}+\mu \boldsymbol{X}^{\prime} \mid \boldsymbol{X}_{\mathrm{L}}, \boldsymbol{X}_{\mathrm{U}}\right)
$$

$S_{\Xi}\left(\boldsymbol{X} \mid \boldsymbol{X}_{\mathrm{L}}, \boldsymbol{X}_{\mathrm{U}}\right)$ is a concave function on $\Xi$ (Lieb and Yngvason, 1999, Thm. 2.8).

(A.13) can be regarded as a mapping $\lambda=\Lambda(\boldsymbol{X})$ from $\Xi$ to $\mathbb{R}$ which also provides a definition of local entropy. It is many-one since if $\lambda=\Lambda(\boldsymbol{X})$ is satisfied by $\boldsymbol{X}$ and $\lambda$ it is also satisfied by all $\boldsymbol{X}^{\prime} \in \partial \mathscr{F}_{\boldsymbol{X}}$ for the same $\lambda$; the boundaries $\partial \mathscr{F} \boldsymbol{X}$ of forward sectors are adiabats (with respect to the local entropy and any entropy WAT-equal to it).

The next stage is to use this local entropy to define an entropy function $S(\boldsymbol{X})$ for all the systems of the family which is independent of a particular choice of $\boldsymbol{X}_{\mathrm{L}}$ and $\boldsymbol{X}_{\mathrm{U}}$ in any $\Xi$. This is done by way of a reflexive, symmetric and transitive relationship of calibration between systems (Lieb and Yngvason, 1999, Thm. 4.7). A single system $\Xi_{\mathrm{Z}}$ is then chosen to be the calibrator for the whole family of systems and in the local entropy the single system is replaced by the Cartesian product $\Xi \times \Xi_{\mathrm{Z}}$, with $\boldsymbol{X}_{\mathrm{L}}, \boldsymbol{X}_{\mathrm{U}} \in \Xi$, replaced, respectively by $\left(\tilde{\boldsymbol{X}}, \boldsymbol{X}_{\mathrm{ZL}}\right)$ and $\left(\tilde{\boldsymbol{X}}, \boldsymbol{X}_{\mathrm{ZU}}\right)$, where $\tilde{\boldsymbol{X}} \in \Xi$ and $\boldsymbol{X}_{\mathrm{ZL}}, \boldsymbol{X}_{\mathrm{ZU}} \in \Xi_{\mathrm{Z}}$ and $\boldsymbol{X}$ replaced by $\left(\boldsymbol{X}, \boldsymbol{X}_{\mathrm{ZL}}\right)$. Thus we have (Lieb and Yngvason, 1999, p. 31)

$$
S(\boldsymbol{X}):=S_{\Xi \times \Xi_{\mathrm{Z}}}\left(\left(\boldsymbol{X}, \boldsymbol{X}_{\mathrm{ZL}}\right) \mid\left(\tilde{\boldsymbol{X}}, \boldsymbol{X}_{\mathrm{ZL}}\right),\left(\tilde{\boldsymbol{X}}, \boldsymbol{X}_{\mathrm{ZU}}\right)\right) .
$$

It can then be shown not only that $S(\boldsymbol{X})$ satisfies (A.14), meaning that it is WAT-equal to the local entropy for any $\Xi$, but that it is concave on $\Xi$ and satisfies the scaling and extensivity conditions (A.6) and (A.7) (Lieb and Yngvason, 1999, Thm. 2.5). Also, from (A.13), $S(\tilde{\boldsymbol{X}})=0$ sets the zero point for the entropy in each $\Xi$.

\section{B. Supporting Material for Sect. 4.2}

For a vector set of functions $\boldsymbol{\xi}(\boldsymbol{X}):=\left(\left(\xi^{(0)}(\boldsymbol{X}), \xi^{(1)}(\boldsymbol{X}), \ldots, \boldsymbol{\xi}^{(n)}(\boldsymbol{X})\right)\right)$, let $\mathcal{D}(\boldsymbol{\xi}):=\boldsymbol{\xi}(\boldsymbol{X}) \cdot \mathbf{d} \boldsymbol{X}$. Then:

Definition B.1. $\mathcal{D}(\boldsymbol{\xi})$ is an exact differential if $\exists \sigma(\boldsymbol{X})$ such that $\boldsymbol{\xi}(\boldsymbol{X})=\boldsymbol{\nabla} \sigma(\boldsymbol{X})$ and hence that $\mathcal{D}(\boldsymbol{\xi})=\mathrm{d} \sigma$.

Definition B.2. The curve $\mathscr{L}\left(\boldsymbol{X}_{0}, \boldsymbol{X}_{1}\right) \subset \Xi$ from $\boldsymbol{X}_{0}$ to $\boldsymbol{X}_{1}$ is $\mathcal{D}(\boldsymbol{\xi})$-conforming if every differential element $\mathbf{d} \boldsymbol{X}$ of the curve satisfies $\mathcal{D}(\boldsymbol{\xi})=0$.

The following result is due to Carathéodory (1935, p. 240):

Theorem B.1. If in every neighbourhood $\mathscr{N}_{\boldsymbol{x}}$ of a given $\boldsymbol{X} \in \Xi$ there exist points that cannot be reached along a piecewise smooth $\mathcal{D}(\boldsymbol{\xi})$-conforming curve then there exists a function $\lambda(\boldsymbol{X})$ making $\mathcal{D}(\boldsymbol{\xi} / \lambda)$ an exact differential.

It was, however, pointed out by Bernstein (1960) that the use of this theorem by Carathéodory is incomplete, since the local nature of Thm. B.1 is not fully taken into account. He proposed and proved the modified (local) theorem that: 
Theorem B.2. If

$$
\boldsymbol{\xi}(\boldsymbol{X}) \cdot \boldsymbol{\xi}(\boldsymbol{X})>0, \quad \forall \boldsymbol{X} \in \Xi
$$

then, given $\boldsymbol{X}_{0} \in \Xi$,

(i) In every (open) neighbourhood $\mathscr{N}_{\mathbf{x}_{0}}$ of $\boldsymbol{X}_{0}, \exists \boldsymbol{X}^{\prime}$ which cannot be joined to $\boldsymbol{X}_{0}$ by a piecewise smooth $\mathcal{D}(\boldsymbol{\xi})$ conforming curve lying entirely in $\mathscr{N}_{\mathbf{x}_{0}}$.

iff

(ii) In some neighbourhood of $\boldsymbol{X}_{0}$ there is a function $\lambda(\boldsymbol{X})$ such that $\mathcal{D}(\boldsymbol{\xi} / \lambda)$ is an exact differential.

\section{Results for the Continuous-Energy System}

Using

$$
\frac{\partial \mathcal{H}(\boldsymbol{x} ; X)}{\partial \zeta} \delta^{\operatorname{Dr}}(E-\mathcal{H}(\boldsymbol{x} ; X))=-\frac{\partial \Theta(E-\mathcal{H}(\boldsymbol{x} ; X))}{\partial \mathcal{H}} \frac{\partial \mathcal{H}(\boldsymbol{x} ; X)}{\partial \zeta}=-\frac{\partial \Theta(E-\mathcal{H}(\boldsymbol{x} ; X))}{\partial \zeta},
$$

where $\zeta:=X$, or $x_{i}$, one of the components of $x$, and (10.3),

$$
\int_{\Gamma} \frac{\partial \mathcal{H}(\boldsymbol{x} ; X)}{\partial X} \delta^{\mathrm{Dr}}(E-\mathcal{H}(\boldsymbol{x} ; X)) \mathrm{d} \Gamma=-\int_{\Gamma} \frac{\partial \Theta(E-\mathcal{H}(\boldsymbol{x} ; X))}{\partial X} \mathrm{~d} \Gamma=-\gamma \frac{\partial \Omega(E, X)}{\partial X}
$$

and

$$
\begin{aligned}
\int_{\Gamma} x_{j} \frac{\partial \mathcal{H}(\boldsymbol{x} ; X)}{\partial x_{i}} \delta^{\operatorname{Dr}}(E-\mathcal{H}(\boldsymbol{x} ; X)) \mathrm{d} \Gamma & =-\int_{\Gamma} x_{j} \frac{\partial \Theta(E-\mathcal{H}(\boldsymbol{x} ; X))}{\partial x_{i}} \mathrm{~d} \Gamma \\
& =\delta^{\mathrm{Kr}}(i-j) \int_{\Gamma} \Theta(E-\mathcal{H}(\boldsymbol{x} ; X)) \mathrm{d} \Gamma-\int_{\Gamma} \frac{\partial\left[x_{j} \Theta(E-\mathcal{H}(\boldsymbol{x} ; X))\right]}{\partial x_{i}} \mathrm{~d} \Gamma \\
& =\delta^{\mathrm{Kr}}(i-j) \gamma \Omega(E, X)-\int_{\Gamma} \frac{\partial\left[x_{j} \Theta(E-\mathcal{H}(\boldsymbol{x} ; X))\right]}{\partial x_{i}} \mathrm{~d} \Gamma .
\end{aligned}
$$

Consider now two systems $\Xi_{A}$ and $\Xi_{B}$ which, as described in Sect. 12.1, are thermally joined to form the system $\Xi_{A B}$ with fixed total energy $E_{\mathrm{T}}$ and ${ }^{75}$

$$
\mathcal{H}_{\mathrm{AB}}\left(\boldsymbol{x}_{\mathrm{A}}, \boldsymbol{x}_{\mathrm{B}} ; X_{\mathrm{A}}, X_{\mathrm{B}}\right):=\mathcal{H}_{\mathrm{A}}\left(\boldsymbol{x}_{\mathrm{A}} ; X_{\mathrm{A}}\right)+\mathcal{H}_{\mathrm{B}}\left(\boldsymbol{x}_{\mathrm{B}} ; X_{\mathrm{B}}\right) .
$$

Then

$$
\begin{aligned}
\delta^{\operatorname{Dr}}\left(E_{\mathrm{T}}-\mathcal{H}_{\mathrm{A}}\left(\boldsymbol{x}_{\mathrm{A}} ; X_{\mathrm{A}}\right)-\mathcal{H}_{\mathrm{B}}\left(\boldsymbol{x}_{\mathrm{B}} ; X_{\mathrm{B}}\right)\right) \\
=\int_{0}^{E_{\mathrm{T}}} \mathrm{d} E_{\mathrm{A}} \int_{0}^{E_{\mathrm{T}}} \mathrm{d} E_{\mathrm{B}} \delta^{\operatorname{Dr}}\left(E_{\mathrm{A}}-\mathcal{H}_{\mathrm{A}}\left(\boldsymbol{x}_{\mathrm{A}} ; X_{\mathrm{A}}\right)\right) \delta^{\operatorname{Dr}}\left(E_{\mathrm{B}}-\mathcal{H}_{\mathrm{B}}\left(\boldsymbol{x}_{\mathrm{B}} ; X_{\mathrm{B}}\right)\right) \delta^{\operatorname{Dr}}\left(E_{\mathrm{T}}-E_{\mathrm{A}}-E_{\mathrm{B}}\right),
\end{aligned}
$$

giving from (11.13),

$$
\begin{aligned}
\omega_{\mathrm{AB}}\left(E_{\mathrm{T}}, X_{\mathrm{A}}, X_{\mathrm{B}}\right) & =\int_{0}^{E_{\mathrm{T}}} \mathrm{d} E_{\mathrm{A}} \int_{0}^{E_{\mathrm{T}}} \mathrm{d} E_{\mathrm{B}} \omega_{\mathrm{A}}\left(E_{\mathrm{A}}, X_{\mathrm{A}}\right) \omega_{\mathrm{B}}\left(E_{\mathrm{B}}, X_{\mathrm{B}}\right) \delta^{\operatorname{Dr}}\left(E_{\mathrm{T}}-E_{\mathrm{A}}-E_{\mathrm{B}}\right) \\
& =\int_{0}^{E_{\mathrm{T}}} \mathrm{d} E_{\mathrm{A}} \omega_{\mathrm{A}}\left(E_{\mathrm{A}}, X_{\mathrm{A}}\right) \omega_{\mathrm{B}}\left(E_{\mathrm{T}}-E_{\mathrm{A}}, X_{\mathrm{B}}\right) .
\end{aligned}
$$

\footnotetext{
${ }^{75}$ This result can be readily generalized to the case where the one deformation variable for the total system is $X_{\mathrm{A}}+X_{\mathrm{B}}$, implying a mechanical as well as a thermal interaction.
} 
From (10.4),

$$
\begin{aligned}
v_{\mathrm{AB}}\left(E_{\mathrm{T}}, X_{\mathrm{A}}, X_{\mathrm{B}}\right) & =\int_{0}^{E_{\mathrm{T}}} \mathrm{d} E_{\mathrm{A}} \omega_{\mathrm{A}}\left(E_{\mathrm{A}}, X_{\mathrm{A}}\right) v_{\mathrm{B}}\left(E_{\mathrm{T}}-E_{\mathrm{A}}, X_{\mathrm{B}}\right)+\omega_{\mathrm{A}}\left(0+, X_{\mathrm{A}}\right) \omega_{\mathrm{B}}\left(E_{\mathrm{T}}, X_{\mathrm{B}}\right), \\
\Omega_{\mathrm{AB}}\left(E_{\mathrm{T}}, X_{\mathrm{A}}, X_{\mathrm{B}}\right) & =\int_{0}^{E_{\mathrm{T}}} \mathrm{d} E_{\mathrm{A}} \omega_{\mathrm{A}}\left(E_{\mathrm{A}}, X_{\mathrm{A}}\right) \Omega_{\mathrm{B}}\left(E_{\mathrm{T}}-E_{\mathrm{A}}, X_{\mathrm{B}}\right),
\end{aligned}
$$

and

$$
\begin{aligned}
\Omega_{\mathrm{AB}}\left(E_{\mathrm{A}}+E_{\mathrm{B}}, X_{\mathrm{A}}, X_{\mathrm{B}}\right) & =\int_{0}^{\left(E_{\mathrm{A}}+E_{\mathrm{B}}\right)} \mathrm{d} E_{\mathrm{A}}^{\prime} \omega_{\mathrm{A}}\left(E_{\mathrm{A}}^{\prime}, X_{\mathrm{A}}\right) \Omega_{\mathrm{B}}\left(E_{\mathrm{A}}+E_{\mathrm{B}}-E_{\mathrm{A}}^{\prime}, X_{\mathrm{B}}\right) \\
& >\int_{0}^{E_{\mathrm{A}}} \mathrm{d} E_{\mathrm{A}}^{\prime} \omega_{\mathrm{A}}\left(E_{\mathrm{A}}^{\prime}, X_{\mathrm{A}}\right) \Omega_{\mathrm{B}}\left(E_{\mathrm{A}}+E_{\mathrm{B}}-E_{\mathrm{A}}^{\prime}, X_{\mathrm{B}}\right) \\
& >\int_{0}^{E_{\mathrm{A}}} \mathrm{d} E_{\mathrm{A}}^{\prime} \omega_{\mathrm{A}}\left(E_{\mathrm{A}}^{\prime}, X_{\mathrm{A}}\right) \Omega_{\mathrm{B}}\left(E_{\mathrm{B}}, X_{\mathrm{B}}\right)=\Omega_{\mathrm{A}}\left(E_{\mathrm{A}}, X_{\mathrm{A}}\right) \Omega_{\mathrm{B}}\left(E_{\mathrm{B}}, X_{\mathrm{B}}\right) .
\end{aligned}
$$

The strict inequalities in this result are important for the discussion in Sect. 16 and arise from the positivity of the integrand and the fact that $\Omega_{\mathrm{B}}\left(E, X_{\mathrm{B}}\right)$ is a monotonically increasing function of $E$.

Suppose now that the flow $\phi_{t}$ is a group of automorphisms determined by Hamilton's equations with $\boldsymbol{x}:=(\boldsymbol{p}, \boldsymbol{q}) \in \Gamma$, where $\boldsymbol{p}$ and $\boldsymbol{q}$ are the momentum and configuration variables. First consider a path $\mathscr{P} \in \Xi$ given by $(E(\lambda), X(\lambda))$ with $\lambda \in[0,1]$. Now assume that $S=S(E, X)$ is known and invertible with respect to $E$. Then the thermodynamic state of the system can also be specified by points $(S, X) \in \Xi^{\prime}$, and there is a related path $\mathscr{P}^{\prime} \in \Xi^{\prime}$ given by $(S(E(\lambda), X(\lambda)), X(\lambda))$ with $\lambda \in[0,1]$ along which

$$
\frac{\mathrm{d} E}{\mathrm{~d} \lambda}=\frac{\partial E(S, X)}{\partial S} \frac{\mathrm{d} S}{\mathrm{~d} \lambda}+\frac{\partial E(S, X)}{\partial X} \frac{\mathrm{d} X}{\mathrm{~d} \lambda} .
$$

Suppose that the parameter $\lambda$ is related to the time $t$ by $\lambda:=t / \tau$ with $t \in[0, \tau]$. At time $t$ the phase point lies on the energy hypersurface $\Sigma(E(t / \tau), X(t / \tau))$, given by (10.1), and the flow $\phi_{t}$ in $\Gamma$ is no longer autonomous. With the initial value $(\boldsymbol{p}(0), \boldsymbol{q}(0)) \in \Sigma(E(0), X(0))$ the phase point will trace out the path $\mathscr{P}_{\Gamma} \in \Gamma$ terminating at $(\boldsymbol{p}(1), \boldsymbol{q}(1)) \in$ $\Sigma(E(1), X(1))$ and determined by Hamilton's equations

$$
\dot{\boldsymbol{q}}=\boldsymbol{\nabla}_{p} \mathcal{H}(\boldsymbol{p}, \boldsymbol{q} ; X(t / \tau)), \quad \dot{\boldsymbol{p}}=-\boldsymbol{\nabla}_{q} \mathcal{H}(\boldsymbol{p}, \boldsymbol{q} ; X(t / \tau)) .
$$

As $t$ increases in the range $[0, \tau]$

$$
\frac{\mathrm{d} E}{\mathrm{~d} t}=\frac{\mathrm{d} \mathcal{H}}{\mathrm{d} t}=\nabla_{p} \cdot \dot{\boldsymbol{p}}+\nabla_{q} \cdot \dot{\boldsymbol{q}}+\frac{\partial \mathcal{H}}{\partial X} \frac{\mathrm{d} X}{\mathrm{~d} t}=\frac{\partial \mathcal{H}}{\partial X} \frac{\mathrm{d} X}{\mathrm{~d} t} .
$$

The only microscopic phase dependence in (C.12) is in the Hamiltonian. We can therefore take its microcanonical expectation to give

$$
\frac{\mathrm{d} E}{\mathrm{~d} t}=\left\langle\frac{\partial \mathcal{H}(\boldsymbol{p}, \boldsymbol{q} ; X)}{\partial X} \mid E, X\right\rangle \frac{\mathrm{d} X}{\mathrm{~d} t} .
$$

From (C.10) and (C.13),

$$
\frac{\partial E(S, X)}{\partial X} \frac{\mathrm{d} X}{\mathrm{~d} t}=\left\langle\frac{\partial \mathcal{H}(\boldsymbol{p}, \boldsymbol{q} ; X)}{\partial X} \mid E, X\right\rangle \frac{\mathrm{d} X}{\mathrm{~d} t}-\frac{\partial E(S, X)}{\partial S} \frac{\mathrm{d} S}{\mathrm{~d} t} .
$$

If the path $\mathscr{P}^{\prime}$ in $\Xi^{\prime}$ lies on a surface of constant entropy

$$
\frac{\partial E(S, X)}{\partial X}=\left\langle\frac{\partial \mathcal{H}(\boldsymbol{p}, \boldsymbol{q} ; X)}{\partial X} \mid E, X\right\rangle .
$$

Consider now a function $W(E, X)$ on $\Xi$. 
- Along the path $\mathscr{P}^{\prime}$ in $\Xi^{\prime}$, lying on a surface of constant entropy, from (C.15),

$$
\frac{\mathrm{d} W}{\mathrm{~d} t}=\frac{\mathrm{d} X}{\mathrm{~d} t}\left\{\frac{\partial W}{\partial E} \frac{\partial E(S, X)}{\partial X}+\frac{\partial W}{\partial X}\right\}=\frac{\mathrm{d} X}{\mathrm{~d} t}\left\{\frac{\partial W}{\partial E}\left\langle\frac{\partial \mathcal{H}(\boldsymbol{p}, \boldsymbol{q} ; X)}{\partial X} \mid E, X\right\rangle+\frac{\partial W}{\partial X}\right\} .
$$

- Along the path $\mathscr{P}_{\Gamma}$ in $\Gamma, W(E, X)$ will vary, with $E(t / \tau)=\mathcal{H}(\boldsymbol{p}, \boldsymbol{q} ; X(t / \tau))$ varying as the Hamiltonian varies and $X$ varying according as $X(t / \tau)$ varies. Then, from (C.13),

$$
\frac{\mathrm{d} W}{\mathrm{~d} t}=\frac{\partial W}{\partial E} \frac{\mathrm{d} E}{\mathrm{~d} t}+\frac{\partial W}{\partial X} \frac{\mathrm{d} X}{\mathrm{~d} t}=\frac{\mathrm{d} X}{\mathrm{~d} t}\left\{\frac{\partial W}{\partial E}\left\langle\frac{\partial \mathcal{H}(\boldsymbol{p}, \boldsymbol{q} ; X)}{\partial X} \mid E, X\right\rangle+\frac{\partial W}{\partial X}\right\} .
$$

It follows from (C.16) that a function $W(E, X)$ is constant along a path on an adiabatic surface iff

$$
\frac{\partial W}{\partial X}=-\frac{\partial W}{\partial E}\left\langle\frac{\partial \mathcal{H}(\boldsymbol{p}, \boldsymbol{q} ; X)}{\partial X} \mid E, X\right\rangle .
$$

Such a function could be called an adiabatic invariant in the thermodynamic sense. On the other hand a global constant of motion $Z(\boldsymbol{p}, \boldsymbol{q} ; X(t / \tau))$ is an adiabatic invariant in the mechanical sense if it is conserved along almost all paths $\mathscr{P}_{\Gamma}$ asymptotically in the limit $\tau \rightarrow \infty$ (Kasuga, 1961). It follows from (C.17) that $W(E, X)$, where the dependence on $\boldsymbol{p}$ and $\boldsymbol{q}$ is through $E=\mathcal{H}(\boldsymbol{p}, \boldsymbol{q} ; X)$, is such an adiabatic invariant in this sense if it satisfies (C.18).

\section{The Perfect Fluid}

In this case the thermodynamic relationship (8.1) for a fluid in a three-dimensional cubic box $\mathscr{V}$ of side $L$ takes the form

$$
T \mathrm{~d} S=\mathrm{d} U+P \mathrm{~d} V
$$

where $V:=L^{3}$ and $P$ are respectively the volume of $\mathscr{V}$ and the pressure of the fluid. The microstate of such a system of $N$ particles of mass $m$ and chemical potential $\mu$ is $\boldsymbol{x}:=(\boldsymbol{p}, \boldsymbol{q}) \in \Gamma$, where $\boldsymbol{p}$ and $\boldsymbol{q}$ are the $3 N$-dimensional momentum and configuration vectors, respectively, with the components of $\boldsymbol{q}$ restricted to the range $\left[-\frac{1}{2} L, \frac{1}{2} L\right]$. The number of particles $N$ is fixed as the size parameter and $V$ is the sole deformation variable. The Hamiltonian is implicitly dependent on the configuration vector $\boldsymbol{q}$, and hence on the volume $V$, through the restriction of its range, and explicitly on the momentum vector in the form $\mathcal{H}(\boldsymbol{p} ; V ; N):=\boldsymbol{p}^{2} /(2 m)$. The phase space element factors in the form $\mathrm{d} \Gamma:=\mathrm{d} \Gamma_{q} \mathrm{~d} \Gamma_{p}$ and it is not difficult to show that

$$
\mathrm{d} \Gamma_{p}=\frac{\mathrm{d} \Sigma_{E} \mathrm{~d} E}{|\nabla \mathcal{H}(\boldsymbol{p} ; V ; N)|}=\sqrt{\frac{m}{2 E}} \mathrm{~d} \Sigma_{E} \mathrm{~d} E,
$$

where $\mathrm{d} \Sigma_{E}$ is the element of the energy surface $\Sigma(E ; N)$ given by (10.1). The area of this $3 N$-dimensional hypersphere is $2\{2 \pi m E\}^{\frac{3}{2} N} /\left[\Gamma\left(\frac{3}{2} N\right) \sqrt{2 m E}\right]$ and, from (10.2), (10.3), (11.1) and (11.5),

$$
\begin{gathered}
\omega(E, V ; N)=\frac{3}{2} N E^{\frac{3}{2} N-1}[K(V ; N)]^{N}, \quad \Omega(E, V ; N)=E^{\frac{3}{2} N}[K(V ; N)]^{N}, \\
S_{\omega}(E, V ; \eta, N)=\ln \left(\frac{3 N \eta}{2 E}\right)+\frac{3}{2} N \ln (E)+N \ln [K(V ; N)], \quad S_{\Omega}(E, V ; N)=\frac{3}{2} N \ln (E)+N \ln [K(V ; N)],
\end{gathered}
$$

where

$$
K(V ; N):=V\left\{\frac{(2 \pi m)^{\frac{3}{2} N}}{h^{3 N} \Gamma(N+1) \Gamma\left(\frac{3}{2} N+1\right)}\right\}^{1 / N} .
$$

For this model $E$ is the kinetic energy of the particles, lying in the range $[0, \infty)$, and the surface and bulk temperatures

$$
T_{\omega}(E, V ; N)=E\left(\frac{3}{2} N-1\right)^{-1}, \quad T_{\Omega}(E, V ; N)=E\left(\frac{3}{2} N\right)^{-1},
$$


are positive, with heat capacities

$$
C_{\omega}(E, V ; N)=\left(\frac{\partial T_{\omega}(E, V ; N)}{\partial E}\right)^{-1}=\frac{3}{2} N-1, \quad C_{\Omega}(E, V ; N)=\left(\frac{\partial T_{\Omega}(E, V ; N)}{\partial E}\right)^{-1}=\frac{3}{2} N,
$$

and, from (D.1), pressures

$$
P_{\omega}(E, V ; N)=\frac{2 N E}{V(3 N-2)}, \quad P_{\Omega}(E, V ; N)=\frac{2 E}{3 V} .
$$

Suppose that $x_{i}$ and $x_{j}$ are any two components of the momenta of any of the particles of the fluid. Then $x_{j} \frac{\partial \mathcal{H}(p ; V ; N)}{\partial x_{i}}=$ $x_{j} x_{i} / m$. The momentum of the $k$-th particle can be expressed in terms of the Euler angles $\theta^{(k)} \in[0,2 \pi], \phi^{(k)} \in[0, \pi]$ as $\boldsymbol{p}^{(k)}:=p^{(k)}\left(\cos \left(\theta^{(k)}\right), \sin \left(\theta^{(k)}\right) \cos \left(\phi^{(k)}\right), \sin \left(\theta^{(k)}\right) \sin \left(\phi^{(k)}\right)\right)$. Thus, in computing the microcanonical expectation value in (11.16) for $i \neq j$, integrations will occur over the periods of harmonic functions yielding a result of zero. In the case $i=j$

$$
\left\langle x_{i} \frac{\partial \mathcal{H}(\boldsymbol{p} ; V ; N)}{\partial x_{i}} \mid E, V ; N\right\rangle=\frac{1}{m}\left\langle x_{i}^{2} \mid E, V ; N\right\rangle=\frac{2}{3 N}\langle E \mid E, V ; N\rangle=\frac{2}{3 N} E=T_{\Omega}(E, V ; N),
$$

showing, from (11.16) that $\mathscr{B}_{i j}(E, V ; N)$, given by (11.17), is, in the case of the perfect fluid, zero for all $i$ and $j$.

From (12.11) and (D.3), the canonical partition function is

$$
Z_{\mathrm{C}}(T, V ; N)=\frac{3}{2} N V^{N}[K(V ; N)]^{N} \int_{0}^{\infty} E^{\frac{3}{2} N-1} \exp (-E / T) \mathrm{d} E=\Gamma\left(\frac{3}{2} N+1\right) T^{\frac{3}{2} N}[K(V ; N)]^{N}
$$

and, from (12.13) and Table 3,

$$
S_{\mathrm{C}}(T, V ; N)=N \ln [K(V ; N)]+\ln \left[\Gamma\left(\frac{3}{2} N+1\right)\right]+\frac{3}{2} N \ln (T)+\frac{3}{2} N
$$

with

$$
\begin{aligned}
& S_{\mathrm{C}}\left(T_{\omega}(E, V ; N), V ; N\right)=\frac{3}{2} N \ln (E)+N \ln [K(V ; N)]+\ln \left[\Gamma\left(\frac{3}{2} N+1\right)\right]+\frac{3}{2} N\left\{1-\ln \left(\frac{3}{2} N-1\right)\right\} . \\
& S_{\mathrm{C}}\left(T_{\Omega}(E, V ; N), V ; N\right)=\frac{3}{2} N \ln (E)+N \ln [K(V ; N)]+\ln \left[\Gamma\left(\frac{3}{2} N+1\right)\right]+\frac{3}{2} N\left\{1-\ln \left(\frac{3}{2} N\right)\right\} .
\end{aligned}
$$

The expectation value of $E$ in the canonical distribution is $\langle E \mid T, V ; N\rangle=\frac{3}{2} N T$, so that

$$
T_{\omega}(\langle E \mid T, V ; N\rangle, V ; N)=\left(\frac{3 N}{3 N-2}\right) T, \quad T_{\Omega}(\langle E \mid T, V ; N\rangle, V ; N)=T .
$$

In the thermodynamic limit when $N \rightarrow \infty, V \rightarrow \infty$, with $N / V$ finite,

$$
\ln \left[\Gamma\left(\frac{3}{2} N+1\right)\right] \stackrel{\mathrm{TL}}{\simeq} \frac{3}{2} N\left\{\ln \left(\frac{3}{2} N\right)-1\right\} \quad \text { and } \quad \ln [K(V ; N)] \stackrel{\mathrm{TL}}{=} \ln (V)+\frac{3}{2} \ln \left(\frac{4 \pi m}{3 h^{2}}\right)-2 \ln (N) .
$$

$S_{\omega}(E, V ; N) \stackrel{\mathrm{TL}}{\simeq} S_{\Omega}(E, V ; N) \stackrel{\mathrm{IL}}{\simeq} S_{\mathrm{C}}\left(T_{\omega}(E, V ; N), V ; N\right) \stackrel{\mathrm{TL}}{\simeq} S_{\mathrm{C}}\left(T_{\Omega}(E, V ; N), V ; N\right)$ give the Sackur-Tetrode equation for the entropy of a perfect fluid (Huang, 1963, Sect. 6.5), with $T_{\omega}(E, V ; N) \stackrel{\text { LL }}{=} T_{\Omega}(E, V ; N)$.

\section{Acknowledgments}

I am very grateful to Roman Frigg for reading and commenting on earlier versions of this work and to Reimer Kühn for many valuable discussions. I also thank the reviewers of the previous versions for their detailed criticisms.

\section{References}


Abragam, A., Proctor, W. G., 1957. Experiments on spin temperatures. Phys. Rev. 106, 160-161.

Abragam, A., Proctor, W. G., 1958. Spin temperature. Phys. Rev. 109, 1441-1458.

Abrahm, E., Penrose, O., 2017. Physics of negative absolute temperatures. Phys. Rev. E 95, 012125-1 012125-8.

Batterman, R. W., 2010. Reduction and renormalization. In: Ernst, G., Hüttemann, A. (Eds.), Time, Chance and Reduction. C.U.P., London, U.K.

Bernstein, B., 1960. Proof of Carathéodory's local theorem and its global application to thermostatics. J. Math. Phys. 1, $222-224$.

Boltzmann, L., 1877. Über die Beziehung zwisschen dem zweiten Hauptsatze der mechanischen Wärmtheorie und der Wahrscheinlichkeitsrechnung resp. den Sätzen über das Wärmeglichgewicht. Weiner Berichte 76, 373-435, reprinted in Boltzmann (1909), 164-223 (page references refer to this reprint).

Boltzmann, L., 1909. Wissenschaftliche Abhandlungen, Vol. II. Leipzig: Barth.

Boyling, J. B., 1972. An axiomatic approach to classical thermodynamics. Proc. Roy. Soc. A 329, 35-70.

Braun, S., Ronzheimer, J. P., Schreiber, M., Hodgman, S. S., Rom, T., Bloch, I., Schneider, U., 2013. Negative absolute temperature for motional degrees of freedom. Science 339, 52-55.

Brown, H. R., Uffink, J., 2001. The origins of time-asymmetry in thermodynamics: the minus first law. Stud. Hist. Phil. Mod. Phys., 32, 525-538.

Brush, S. G., 1976. The Kind of Motion We Call Heat. Vol. 2: Statistical Physics and Irreversible Processes. North-Holland Pub. Co.

Buchdahl, H. A., 1966. The Concepts of Classical Thermodynamics. C.U.P. London.

Buonsante, P., Franzosi, R., Smerzi, A., 2016. On the dispute between Boltzmann and Gibbs entropy. Am. Phys. 375, $414-434$.

Callen, H. B., 1985. Thermodynamics and an Introduction to Thermostatics, 2nd Edition. Wiley, New York.

Campisi, M., 2015. Construction of microcanonical entropy on thermodynamic pillars. Phys. Rev. E 91, 052147-1-6.

Carathéodory, 1935. Variationsrechnung und Partielle Differentialgleichungen Erste Ordnung. B. G. Teubner, Berlin, page references given to the English translation: Calculus of Variations and Partial Differential Equations of the First Order. Part I, by R. B. Dean and J. J. Brandstatter, Holden-Day Inc. 1965.

Carathéodory, C., 1909. Untersuchungen über die Grundlagen der Thermodynamik. Mathematische Annalen 67, 355-386, page references given to the English translation: Investigation into the Foundations of Thermodynamics, by J. Kestin in The Second Law of Thermodynamics, Benchmark Papers on Energy, Vol. 5, (Ed. J. Kestin), Dowden, Hutchinson and Ross, 1976, pp. 229-256.

Carr, L. D., 2013. Negative temperatures? Science 339, 42-43.

Clausius, R., 1865. Fourth Memoir (1854): On a modified form of the second fundamental theorem. In: The Mechanical Theory of Heat with its Applications to the Steam Engine and to Physical Properties of Bodies. John van Voorst, London, pp. 111-135.

Cooper, J. L. B., 1967. The foundations of thermodynamics. J. Math. An. App. 17, 172-193.

Copson, E. T., 1967. Asymptotic Expansions. C. U. P. London.

Dunkel, J., Hilbert, S., 2014a. Consistent thermostatistics forbids negative absolute temperatures. Nature Physics 10, 67-72, (The online version at: www.physik.uni-augsburg.de \theo1 $\backslash$ hanggi $\backslash$ Dunkel_Nature.pdf includes important supplementary information.).

Dunkel, J., Hilbert, S., 2014b. Reply to Frenkel and Warren, arXiv:1403.6058v1.

Dunkel, J., Hilbert, S., 2014c. Reply to Schneider et al., arXiv:1408.5392v1.

Ehrenfest-Afanassjewa, T., 1925. Zur Axiomatisierung des zweiten Hauptsatzes der Thermodynamik. Zeitschrift für Physik 33, $933-945$.

Emch, G., Liu, C., 2002. The Logic of Thermostatistical Physics. Springer, Berlin, Heidelberg, Germany.

Frenkel, D., Warren, P. B., 2015. Gibbs, Boltzmann, and negative temperatures. Am. J. Phys. 83, 163-170

Georgii, H.-O., 1995. The equivalence of ensembles of classical systems of particles. J. Stat. Phys. 80, 1341-1378.

Gibbs, J. W., 1902. Elementary Principles in Statistical Mechanics. Yale University Press, reprinted by New York: Dover (1960).

Giles, R., 1964. Mathematical Foundations of Thermodynamics. Pergamon Press, Oxford.

Hakonen, P. J., Vuorinen, R. T., Martikainen, J. E., 1993. Nuclear antiferromagnetism in rhodium metal at positive and negative nanokelvin temperatures. Phys. Rev. Lett. 70, 2818-2821.

Hänggi, P., Hilbert, S., Dunkel, J., 2016. Meaning of temperature in different thermostatical ensembles. Phil. Trans. Roy. Soc. A 10, $374: 2015.0039$.

Hilbert, S., Hänggi, P., Dunkel, J., 2014. Thermodynamic laws in isolated systems. Phys. Rev. E 90, 061161-1 061161-23.

Huang, K., 1963. Statistical Mechanics. John Wiley, New York.

Joule, J. P., 1850. On the mechanical equivalent of heat. Proc. Roy. Soc. London 140, 61-82.

Kasuga, T., 1961. On the adiabatic theorem for the Hamiltonian system of differential equations in the classical mechanics. Proc. Jpn. Acad. 37, $366-382$.

Khinchin, A. I., 1949. Mathematical Foundations of Statistical Mechanics. Dover, New York.

Landsberg, P. T., 1956. Foundations of thermodynamics. Rev. Mod. Phys. 28, 363-392.

Landsberg, P. T., 1961. Thermodynamics. Interscience, New York, London.

Landsberg, P. T., 1977. Heat engines and heat pumps at positive and negative absolute temperatures. J. Phys. A 10, 1773-1780.

Landsberg, P. T., Tykodi, R. J., Tremblay, A.-M., 1980. Systematics of Carnot cycles at positive and negative Kelvin temperatures. J. Phys. A. Math. Gen. 13, 1063-1074.

Lavis, D. A., 2005. Boltzmann and Gibbs: An attempted reconciliation. Stud. Hist. Phil. Mod. Phys. 36, $245-273$.

Lavis, D. A., 2008. Boltzmann, Gibbs and the concept of equilibrium. Philosophy of Science 75, 682-696.

Lavis, D. A., 2015. Equilibrium Statistical Mechanics of Lattice Systems. Springer, Berlin, Heidelberg, New York.

Lavis, D. A., 2018. The problem of equilibrium processes in thermodynamics. Stud. Hist. Phil. Mod. Phy. 62, $136-144$.

Lieb, E. H., Yngvason, J., 1998. A guide to entropy and the second law of thermodynamics. Notices of the Amer. Math. Soc. 45, 571-581.

Lieb, E. H., Yngvason, J., 1999. The physics and mathematics of the second law of thermodynamics. Phys. Rep. 310, 1-96, for Fig. 8 see ibid. (1999), 314, 699.

Lieb, E. H., Yngvason, J., April 2000. A fresh look at entropy and the second law of thermodynamics. Physics Today 53, $32-37$.

Lieb, E. H., Yngvason, J., 2013. The entropy concept for non-equilibrium states. Proc. R. Soc. A 469, 20130408.

Lynden-Bell, D., 1999. Negative specific heat in astronomy, physics and chemistry. Physica A 263, $293-304$.

Marsland, III, R., Brown, H. R., Valente, G., 2015. Time and irreversibility in axiomatic thermodynamics. Am. J. Phys. 83, 628-634.

Martin-Löf, A., 1979. The equivalence of ensembles and the Gibbs phase rule for classical lattice systems. J. Stat. Phys. 20, 557-569. 
Matty, M., Lancaster, L., Griifin, W., Swendsen, R. H., 2017. Comparison of canonical and microcanonical definitions of entropy. Physica A 467, 474-489.

Medley, P., Weld, D. M., Miyake, H., Pritchard, D. E., Ketterle, W., 2011. Spin gradient demagnetization cooling of ultracold atoms. Phys. Rev. Lett. 106, 195301-1-4.

Mosk, A. P., 2005. Atomic gases at negative kinetic temperature. Phys. Rev. Lett. 95, 040403-1-4.

Norton, J. D., 2016. The impossible process: thermodynamic reversibility. Stud. Hist. Phil. Mod. Phys. 55, $43-61$.

Pathria, R. K., 1972. Statistical Mechanics. Pergamon, Oxford.

Pippard, A. B., 1961. The Elements of Classical Thermodynamics. C. U. P. London.

Planck, M., 1903. Treatise on Thermodynamics. Longmans, Grace and Co. Ltd, London, (Translated by A. Ogg).

Poulter, J., 2016. In defense of negative temperature. Phys. Rev. E 93, 032149-1 - 4.

Pound, R. V., 1951. Nuclear spin relaxation times in single crystals of LiF. Phys. Rev. 81, 156.

Prestipino, S., Giaquinta, P. V., 2003. The concavity of entropy and extremum principles in thermodynamics. J. Stat. Phys. 111, $479-493$.

Purcell, E. M., Pound, R. V., 1951. A nuclear spin system at negative temperature. Phys. Rev. 81, 279-280.

Ramsey, N. F., 1956. Thermodynamics and statistical mechanics at negative absolute temperatures. Phys. Rev. 103, 20-28.

Ramsey, N. F., Pound, R. V., 1951. Nuclear audifrequency spectroscopy by resonant heating of the nuclear spin system. Phys. Rev. 81, $278-279$.

Rapp, A., Mandt, S., Rosch, A., 2010. Equilibration rates at negative absolute temperatures for ultracold atoms in optical lattices. Phys. Rev. Lett. 105, 220405-1-4.

Ruelle, D., 1969. Statistical Mechanics. Benjamin, New York.

Schneider, U., Mandt, S., Rapp, A., Braun, S., Weimer, H., Bloch, I., Rosch, A., 2014. Comment on "Consistent thermostatistics forbids negative absolute temperatures", arXiv:1407.4127v1.

Sklar, L., 1993. Physics and Chance. C.U.P. London.

Swendsen, R. H., 2017a. Resolving the debate about proposed expressions for the classical entropy, arXiv.1507.1702.05810v5 [cond-mat-.statmech].

Swendsen, R. H., 2017b. Thermodynamics, statistical mechanics and entropy. Entropy 19, 603-1 - 22.

Swendsen, R. H., 2018. Thermodynamics of finite systems: a key issues review. Rep. Prog. Phys. 81, 072001-1 - 15.

Swendsen, R. H., Wang, J., 2015. Gibbs volume entropy is incorrect. Phys. Rev. E 92, 0201031-1 0201031-5.

Swendsen, R. H., Wang, J., 2016. Negative temperatures and the definition of entropy. Physica A 453, $24-34$.

ter Haar, D., 1961. Elements of Statistical Mechanics. Holt, Rinehart and Winston. New York.

Thomson, W., 1882. On the dynamical theory of heat, with numerical results deduced from Mr. Joule's equivalent of a thermal unit, and M.

Regnault's observations of steam. In: Mathematical and Physical Papers of William Thomson, Vol. 1. C. U. P. London, pp. 174-200.

Tolman, R. C., 1938. The Principles of Statistical Mechanics. O. U. P. Oxford.

Touchette, H., 2011. Ensemble equivalence for general many-body systems. Europhys. Lett. 96, 50010-p1 - p5.

Touchette, H., 2015. Equivalence and nonequivalence of ensembles: thermodynamic, macrostate, and measure levels. J. Stat. Phys. 159, 987-1016.

Tremblay, A.-M., 1976. Comment on "Negative Kelvin temperatures: Some anomalies and speculation.”. Am. J. Phys. 44, 994-995.

Tykodi, R. J., 1978. Quasi-Carnot cycles, negative Kelvin temperatures and the laws of thermodynmics. Am. J. Phys. 46, 354-359.

Uffink, J., 2001. Bluff your way in the second law of thermodynamics. Stud. Hist. Phil. Mod. Phys. 32, 305-394.

Vilar, J. M. G., Rubi, J. M., 2014. System size scaling of Boltzmann and alternate Gibbs entropies. J. Chem. Phys. 140, 201101-1-3.

Wallace, D., 2014. Thermodynamics as control theory. Entropy 16, 699-725.

Wang, G. M., Sevick, E. M. amd Mittag, E., J., S. D., Evans, D. J., 2002. Experimental demonstration of violations of the second law of thermodynamics for small systems and short time scales. Phys. Rev. Lett. 89, 050601-1 - 4 .

Wang, J., 2015. Critique of the Gibbs volume entropy and its implications, arXiv:1507.02022v1. 